3D printed sensing systems for upper extremity assessment 


\section{D PRINTED SENSING SYSTEMS FOR UPPER EXTREMITY ASSESSMENT}

Gerjan Wolterink 


\section{Graduation committee}

Chairman and secretary

prof. dr. J.N. Kok

University of Twente

Supervisors

prof. dr. ir. G.J.M. Krijnen University of Twente

prof. dr. ir. P.H. Veltink

University of Twente

Co-supervisor

dr. ir. B.J.F. Van Beijnum University of Twente

Committee members

prof. dr. E. MacDonald University of Texas

prof. dr. V.G.M. Weerdesteyn Radboud University

prof. dr. D. Prattichizzo University of Siena

prof. dr. J.H. Buurke University of Twente

prof. dr. ir. M. Sartori University of Twente

This work was supported by the SoftPro project, funded by the European Union's Horizon 2020 research and innovation programme under grant agreement No. 688857.

Printed by: Gildeprint, Enschede

ISBN: $\quad$ 978-90-365-5332-2

DOI: $\quad 10.3990 / 1.9789036553322$

Copyright (C) 2022 by G.J.W. Wolterink, The Netherlands. All rights reserved. No parts of this thesis may be reproduced, stored in a retrieval system or transmitted in any form or by any means without permission of the author. Alle rechten voorbehouden. Niets uit deze uitgave mag worden vermenigvuldigd, in enige vorm of op enige wijze, zonder voorafgaande schriftelijke toestemming van de auteur. 


\title{
3D PRINTED SENSING SYSTEMS FOR UPPER EXTREMITY ASSESSMENT
}

\author{
DISSERTATION
}

to obtain

the degree of doctor at the University of Twente, on the authority of the rector magnificus, prof. dr. ir. A. Veldkamp,

on account of the decision of the Doctorate Board, to be publicly defended

on Friday 11 February 2022 at 14.45 hours

by

\section{Gerhard Jan Willem Wolterink}

born on the $13^{\text {th }}$ of August, 1992

in Almelo, The Netherlands 
This dissertation has been approved by:

Supervisors

prof. dr. ir. G.J.M. Krijnen

prof. dr. ir. P.H. Veltink

Co-supervisor

dr. ir. B.J.F. van Beijnum 


\section{Abstract}

Stroke is one of the main causes of disability in the world, resulting in many cases in the loss of motor function. A proper assessment of motor function is required to help to direct and evaluate therapy. Currently, this assessment is performed by therapists using observer-based assessment protocols on a time to time basis influenced by subjective observations and interpretations. Sensorbased technologies can be used to objectively quantify the presence and severity of motor impairments in stroke patients. The main goal of this thesis is the development of a distributed sensing system for the upper limbs that integrates electromyography (EMG), kinematic and kinetic sensor modalities using soft, flexible and personalized sensing structures, to receive continuous information about the interaction of the human body with the environment. The Kinematic data was obtained using existing inertial measurement units (IMU's). New soft and customizable EMG and kinematic sensors were developed using fused deposition modelling (FDM) 3D-printing technologies.

A minimally obstructive distributed inertial sensing system, intended to measure kinematics of the upper extremity, was developed and tested in a pilot study with 10 chronic stroke subjects performing arm-related tasks from a clinical assessment protocol (FMA-UE) with the affected and non-affected side. This study showed the high potential of this measurement system to assess pathological synergies and may provide more detailed clinical information with respect to observer based assessment protocols. Therefore, this system enables the possibility for a quantitative assessment of the upper limb synergies and improvements in the rehabilitation progress.

The emerging development of 3D-printing technologies and materials facilitate the creation of personalized sensing structures that can be potentially integrated in e.g. prosthetic and assistive devices. This technology is used to develop flexible carbon-black doped TPU-based surface electromyography (sEMG) sensing structures that have equal performance in signal amplitude as equal sized gold standard $\mathrm{Ag} / \mathrm{AgCl}$ gel electrodes. 
Sensors currently used for fingertip interaction force sensing lack compliance to the fingertip tissue and therefore result in loss of touch sensation. Furthermore, poor sensor to skin attachment leads to unwanted movements of the sensors around the fingertip caused by the external forces. To overcome these difficulties a new flexible 3D-printed finger sensor was developed that is compliant to the soft fingertip tissue, while trying keeping the loss of touch sensation low. This sensor measures both shear and normal forces by using the mechanical deformation of the fingertips caused by the normal and shear-forces. A major challenge of these 3D-printed sensors is the nonlinear force to signal relation due to the use of (carbon doped) thermoplastic materials. Therefore, signal analysis and compensation models are needed to obtain a signal response correlated to the applied forces.

Insights gained in this research and the ongoing research on new sensor designs, signal analysis methods and the advent of 3D-printing technologies, will enable the future fabrication of personalized integrated sensing devices for applications in e.g. prosthetics, orthotics or soft robotics. These structures could transfer to clinical applications and potentially improve our knowledge, understanding and assessment of pathological synergies that may eventually enable personalized and targeted rehabilitation activities. 


\section{Samenvatting}

Een beroerte (CVA) is een van de belangrijkste oorzaken van invaliditeit in de wereld en leidt in veel gevallen tot een beperking van de bewegingscontrole. Voor het toepassen van de juiste behandeling gedurende revalidatie therapie en om de resultaten van de therapie te beoordeelden, is er een goede beoordeling van de bewegingscontrole nodig. Momenteel wordt deze beoordeling uitgevoerd door een therapeut met behulp van standaard beoordelingsprotocollen. Het nadeel is dat deze methode gevoelig is voor subjectieve observaties en interpretaties. Nieuwe sensor technologieën maken continue monitoring mogelijk en kunnen worden gebruikt om de aanwezigheid en ernst van motorische stoornissen bij CVA patiënten objectief te kwantificeren. Het hoofddoel van dit proefschrift is de ontwikkeling van een gedistribueerd sensor systeem voor de bovenste extremiteiten dat elektromyografie (EMG), kinematische en kinetische sensor modaliteiten integreert met behulp van zachte, flexibele en gepersonaliseerde sensor structuren om een continue meting van de interactie van het menselijk lichaam met de omgeving mogelijk te maken. De kinematische data werd verkregen met behulp van bestaande inertiële sensoren (IMU's). Nieuwe zachte en personaliseerbare EMG en kinematische sensoren werden ontwikkeld met behulp van Fused Deposition Modeling (FDM) 3D-print technologieën.

Een gedistribueerd sensor systeem bestaande uit inertiële sensoren, bedoeld om de kinematica van de bovenste extremiteiten te meten zonder hinder voor de patiënt, werd ontwikkeld en getest in een pilotstudie met tien CVA patiënten. In deze studie werden arm gerelateerde taken uitgevoerd volgens een klinisch beoordelingsprotocol (FMA-UE) met zowel de aangedane als niet-aangedane zijde. Deze meetsystemen hebben de potentie om pathologische synergieën te beoordelen en tonen mogelijk meer gedetailleerde informatie over de pathologische synergieën dan de huidige beoordelingsprotocollen. Daarom bieden deze sensorsystemen de mogelijkheid tot kwantitatieve beoordeling van de bewegingscontrole en tot een verbeteringen van het revalidatie traject.

De groeiende ontwikkeling van 3D-print technologieën en materialen maken 
het mogelijk om gepersonaliseerde sensorstructuren te maken die geïntegreerd kunnen worden in bijvoorbeeld protheses en andere hulpmiddelen. Deze technologie is gebruikt om flexibele, met koolstof gedoteerd, thermoplastisch polyurethanen oppervlakte-elektromyografie (sEMG) elektroden te ontwikkelen. De signaalamplitude van deze elektroden is vergelijkbaar met die van de gouden standaard $\mathrm{Ag} / \mathrm{AgCl}$-gel elektroden.

De sensoren die momenteel worden gebruikt voor het meten van de interactiekrachten op de vingertoppen zijn stijf en vormen niet mee met de zachte vingertoppen en beperken daarom het tastgevoel van de gebruiker. Daarnaast leidt de slechte hechting van de sensor aan de huid tot ongewenste bewegingen ten gevolge van externe krachten. Om deze problemen te verhelpen werd een nieuwe 3D-geprinte vingersensor ontwikkeld die zich vormt naar het zachte vingertop weefsel om het verlies van tastgevoel te beperken. Deze sensor meet zowel schuif- als normaalkrachten door gebruik te maken van de mechanische vervorming van de vingertoppen veroorzaakt door deze krachten. Een grote uitdaging van deze 3D-geprinte sensoren is de niet-lineaire kracht tot signaal relatie veroorzaakt door het gebruik van (met koolstof gedoteerde) thermoplastische materialen. Door gebruik te maken van signaalanalyse en compensatiemodellen kan een signaalrespons worden verkregen die gecorreleerd is met de uitgeoefende kracht.

De inzichten die zijn opgedaan in dit onderzoek en de voortgaande onderzoeken naar nieuwe sensor ontwerpen, signaalanalyse methoden en de komst van nieuwe 3D-printtechnologieën, zullen in de toekomst de fabricage van gepersonaliseerde geïntegreerde sensor systemen mogelijk maken voor toepassingen in bijvoorbeeld protheses, ortheses en soft robotics. Deze sensorstructuren zullen in de toekomst gebruikt kunnen worden in klinische toepassingen en mogelijk onze kennis, begrip en beoordeling van pathologische synergieën verbeteren en uiteindelijk gepersonaliseerde en gerichte revalidatietherapie mogelijk maken. 



\section{Contents}

1 Introduction $\quad 1$

1.1 Distributed sensing for quantification of pathological synergies . 1

1.2 Development of 3D-printed soft sensors . . . . . . . . . . . . . 4

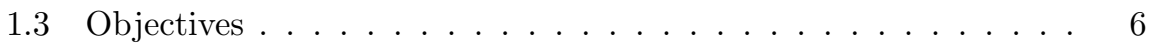

1.4 Thesis outline . . . . . . . . . . . . . . . 7

2 Quantifying pathological synergies in the upper extremity of stroke subjects with the use of IMUs $\quad 9$

2.1 Introduction . . . . . . . . . . . . . . . . . . . 10

2.2 Methods . . . . . . . . . . . . . . . . . 12

2.3 Results......................... 21

2.4 Discussion . . . . . . . . . . . . . . . . . 23

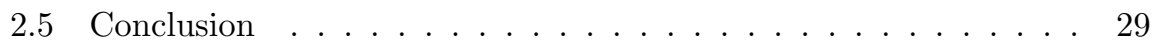

3 Extrusion based 3D printed sensors 31

3.1 Introduction . . . . . . . . . . . . . . . . . . . . 32

3.2 Technologies for extrusion based 3D printed sensors . . . . . . . 33

3.3 Materials . . . . . . . . . . . . . . . . . . 36

3.4 Bonding technology . . . . . . . . . . . . . . . . . . 40

3.5 Anisotropic conduction in 3D printed materials . . . . . . . . . 41

3.6 Compensation methods . . . . . . . . . . . . . . . . 43

3.7 Examples of 3D printed sensors . . . . . . . . . . . . . . . . . . . 43

3.8 Conclusion . . . . . . . . . . . . . . . . 45

$43 \mathrm{D}$ printed soft sEMG sensing structures $\quad 47$

4.1 Introduction . . . . . . . . . . . . . . . . . . . 48

4.2 Methods . . . . . . . . . . . . . . . . . . 52

4.3 Results....................... . . 59

4.4 Discussion . . . . . . . . . . . . . . . 62 


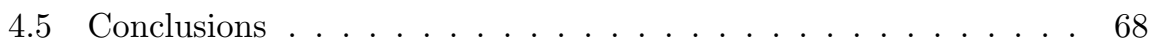

5 A 3D printed soft fingertip sensor for measuring normal and $\begin{array}{ll}\text { shear components of interaction forces } & \mathbf{7 1}\end{array}$

5.1 Introduction . . . . . . . . . . . . . . . . 72

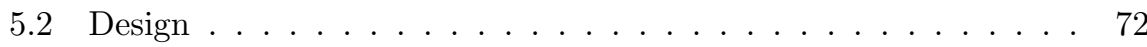

5.3 Numerical Simulation . . . . . . . . . . . . . . . 74

5.4 Methods . . . . . . . . . . . . . . . . . . 75

5.5 Results . . . . . . . . . . . . . . . . . 80

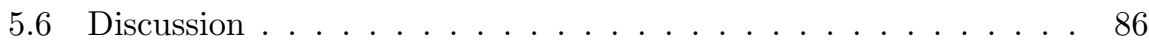

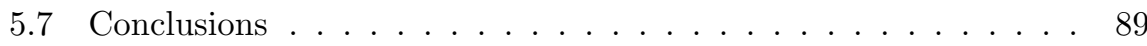

6 Evaluation of a 3D printed soft sensor for measuring fingertip $\begin{array}{ll}\text { interaction forces } & 91\end{array}$

6.1 Introduction . . . . . . . . . . . . . . . . . 92

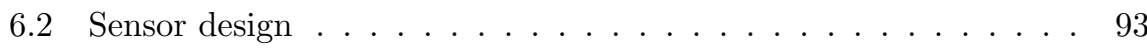

6.3 Methods . . . . . . . . . . . . . . . . . . . 94

6.4 Results . . . . . . . . . . . . . . . . . . . . 100

6.5 Discussion . . . . . . . . . . . . . . . . . . . . . . 108

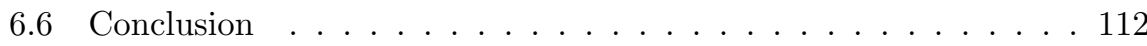

7 Discussion 113

7.1 Motor function assessment using distributed sensing systems . . 114

7.2 Development of 3D printed sensors . . . . . . . . . . 118

8 Conclusion 123

$\begin{array}{ll}\text { Publications } & 147\end{array}$

$\begin{array}{ll}\text { About the author } & 151\end{array}$

$\begin{array}{ll}\text { Dankwoord } & 153\end{array}$ 



\section{Chapter 1 \\ Introduction}

Information about the upper limb synergies is of great value for application in the control of prosthesis, assistive devices and for the quantitative assessment of rehabilitation therapy. In this research, the information of the upper extremities will concentrate on three sensor modalities: electromyography (EMG), kinematic and kinetic. For kinematic data existing inertial measurement units (IMU's) are used. For the EMG and kinetic data new soft and customizable sensors are developed using 3D-printing technologies. The final goal is to create integration of EMG, kinematic and kinetic sensing data, from soft flexible personalized sensing structures, to receive continuous information about the interaction of the human body with the environment.

\subsection{Distributed sensing for quantification of pathologi- cal synergies}

\subsubsection{Stroke and Pathological synergies}

With 80 million stroke survivors and 13.7 million new stroke cases worldwide in 2016, stroke is the third leading disability in the world [1, 2]. A cerebrovascular accident (CVA) or stroke is caused by an interruption of blood flow in the brain, either by a blockage (ischemic) or rupture (haemorrhagic) of the blood vessel [3], resulting in brain tissue damage. This damage can lead to motor impairments typically affecting movement control on one side of the body in the face, arm and legs in about $80 \%$ of patients [4]. Many stroke survivors develop abnormal synergistic movement patterns, still present six months after stroke $[5,6]$. Especially the recovery of arm and hand function lags behind of the recovery [7]. These abnormal synergistic movement patterns at the upper limbs involve a typical muscle co-activation of shoulder abductors together with the 
elbow and wrist flexors, resulting in a reduced range of motion when reaching against gravity [8]. These pathological synergies are strongly affecting activities of daily living of these stroke survivors.

\subsubsection{Assessment of upper limb synergies}

Assessment of the recovery of the upper limb motor function will help to improve the development of effective rehabilitation therapy [6]. Currently, this assessment and the evaluation of the recovery is measured by the observation by a physical therapist on a time to time basis influenced by subjective observations and interpretations. Furthermore, these assessment protocols suffer from ceiling effects and lack fine grain details due to the use of ordinal scales [6, 9]. A quantitative assessment device allows for longer recording and an objective overview of the pathological synergies and the progress of the recovery. Kinematic and kinetic sensors will help to increase the understanding and knowledge of pathological synergies and rehabilitation [10], enabling the possibility to design targeted rehabilitation therapies. Furthermore, in the long run these devices could provide real-time feedback during daily-life recordings to both therapist and patients about the effectiveness of the exercise and allow them to adapt the therapy based on the metrics analysed by the system.

\section{Kinematic sensing}

There exists a variety of systems available for tracking human body kinematics, the gold standard are optical systems [11]. However, these systems have their limitations in the high cost and the need for a customized room with a fixed camera setup. Other options are the use of robotic systems, these are usually used in combination with weight support of the affected limb $[12,13]$. These systems however, tend to be used only in situations with a limited degree of freedom. Inertial measurement units (IMU) are a low-cost and portable solution and often used technology to track human motion in sport, entertainment and healthcare applications [11]. The small size of the units makes them minimally intrusive and, when battery powered, there are no limitations to the workspace.

\section{Kinetic sensing}

Adding information about the interaction forces of the hand during all day activity grasping tasks to kinematic measures, highly improves the clinical relevance of the measurement system $[14,15]$. Since kinetic information is impossible to evaluate by observer based measurement methods, multiple approaches have been taken to capture the interaction forces. One of these approaches is the use of sensorized objects, consisting of faces mounted around a core of force sen- 
sors $[16,17]$. Although those systems give complete and reliable measures, these sensorized objects are limited in the grasping strategies that can be performed and lack the versatility to represent multiple all day tasks. A solution would be to place force sensors on the finger tips, as e.g. demonstrated by Battaglia et al. and Kortier et al. [14, 18]. Although these finger mounted systems offer more flexibility to capture a range of grasping tasks, the use of non compliant materials to the soft fingertip tissue results in loss of touch sensation. Furthermore, due to poor sensor to skin attachment, the sensors tend to shift easily by external forces [18] resulting in limited reliability of the current available systems [19].

\section{EMG}

Electromyography (EMG) is a technique to measure the electrical activity produced by muscles. EMG can be recorded in an invasive manner by using intramuscular electrodes (iEMG), providing fine grain information about individual muscle fibres [20, 21]. More commonly EMG is measured non-invasively by electrodes placed at the surface of the skin. Surface EMG (sEMG) provides information about the global muscle activity and is a widely used technique in gait analysis, biofeedback therapy, fatigue studies and the control of prosthesis $[21,22,23]$. Studying muscle activity of the upper extremities also provides insight in the pathological muscle coupling in stroke subjects by studying muscle co-activation patterns [24], muscle onset times [25] and weakness [26]. These and other EMG metrics might provide more insight in the recovery process and could help to improve therapy.

\section{Integration}

Integrating EMG measurement systems together with the kinematic and kinetic systems allows for an objective overview of the pathological synergies and the progression of the therapy. The Instrumented Arm and Hand Measurement System (iHAMS) (Figure 1.1) is one of the early integrated systems for the upper extremities. This system consists of IMUs placed on the sternum, upper arm, lower-arm, hand, the distal phalanxes of index finger, middle finger and thumb. Fingertip contact force is measured at the fingertips of the index-, middle-finger and thumb. Finally both IMU and force sensor data streams are synchronized with a commercial EMG system. The iHAMS system was developed within the University of Twente, the main hardware architecture is build upon the PowerGlove, a system with IMUs positioned on each finger segment [27]. Systems like the iHAMS system will potentially impact our knowledge, understanding and the assessment of pathological synergies and have the potential to realize more 


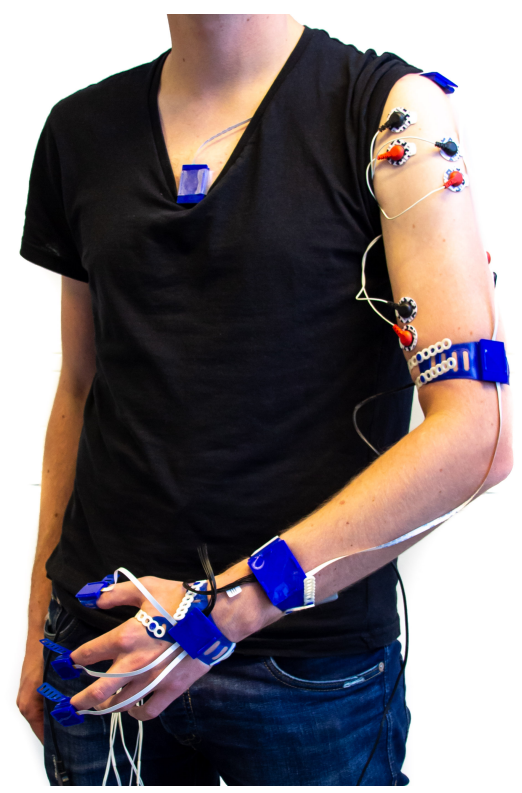

Figure 1.1: The iHAMS system, consisting of IMUs and EMG electrodes placed on the upper extrimities and force sensors on the fingertips.

specific and targeted rehabilitation activities.

\subsection{Development of 3D-printed soft sensors}

Additive manufacturing, of which 3D-printing is the best-known representative, is an emerging technology and has many benefits such as small volume, distributed fabrication, design freedom and customisability. With the growing number of available materials such as soft and conductive materials and with the advent of multi-materials printers, conductive and dielectric materials can be combined. This enables the possibilities to create soft and customizable sensing structures.

\subsubsection{Technology}

There exist numerous 3D-Printing methods making use of different types of technologies such as, but not limited to, vat photopolymerization, sintering, melting, (binder) jetting and extrusion. Direct multi-material printing of conductive materials is currently only commercially possible using extrusion based 

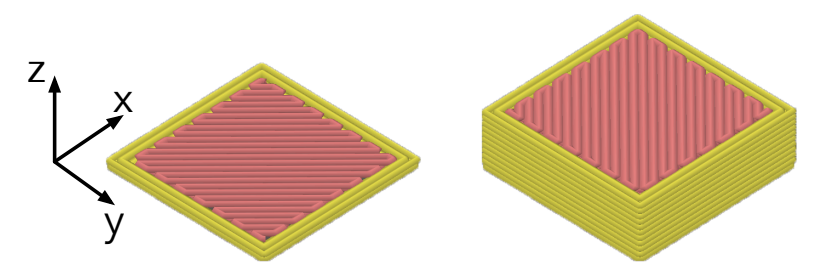

Figure 1.2: Printing path for an FDM printed structure showing the traxels and stacked layers, causing the anisotropy of the material.

technologies were a molten material is deposited in track elements, or traxels, and solidifies [28]. Extrusion based 3D-printing technologies can add conductive materials by depositing conductive ink or paste, adding conductive fibres or by deposition of molten thermoplastics doped with conductive particles. Extrusionbased 3D-printing with molten thermoplastics is commonly known as fused filament fabrication (FFF) or fused deposition modelling (FDM). The conductive materials are deposited in traxels in the $x$ and $y$-directions and stacked layer by layer in the $z$-direction to create height as shown in Figure 1.2. This creates contact interfaces between these lines and layers and therefore, the printed conductive structures are anisotropic [29, 30, 31].

\subsubsection{Printed sensors}

\section{Printed EMG sensors}

Currently, surface electromyography (sEMG) electrodes are only available in fixed shapes. Some electrode structures, such as the TMSi High-Density EMG grids [32] are flexible but cannot be stretched in such a way that complex body shapes are conformally covered by the electrodes. Therefore it is often still necessary to position each electrode individually. For improved everyday patient use of EMG assistive technologies personalized sensing structures are preferable. 3D-printing technologies enable the creation of personalised sensing structures that require minimal assembly and are easily scalable in size and the number of electrodes. The printed structures can be soft and compliant with the soft human tissue, so they do not influence musculoskeletal deformations, greatly enhancing the user's comfort and aesthetics of the device. Furthermore, these electrode structures might include printed wire routing and shielding and can be embedded in other 3D-Printed structures such as prostheses and measurement devices as demonstrated in Figure 1.3. 


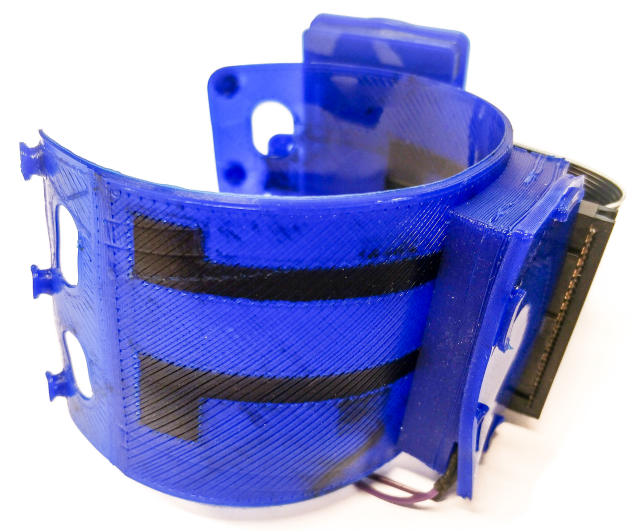

Figure 1.3: 3D-Printed flexible EMG band, the electrodes (in black) are placed according to a specific user and routed to an embedded housing fitting an EMG amplifier and microcontroller for wireless transmission.

\section{Printed force sensors}

The aforementioned benefits of 3D-printing can also be beneficial for the development of sensors to capture interaction forces. Some early FDM printed force sensors have been demonstrated and make use of piezoresistive properties of the flexible conductive polymer composites or use parallel capacitive plates $[33,34,35,36]$. These technologies can be used to fabricate personalized compliant finger interaction force sensing structures. These structures have the potential to limit the issues of the current available non-compliant force sensors.

\subsection{Objectives}

The main goal of this thesis is the development of a distributed sensing system for the upper limbs that integrates EMG, kinematic and kinetic sensing data, using soft, flexible and personalized sensing structures, to receive continuous information about the interaction of the human body with the environment.

More specifically this thesis is focusing on three main research questions covering the three sensor modalities of the envisioned distributed sensing system:

- What are the capabilities of IMU sensing in evaluating motor function 
of the upper limb of stroke patients and comparing the pathological with non-pathological movement patterns?

- What are the possibilities to use 3D-printing for fabrication of customised sEMG sensors and sensor arrays?

- How to fabricate a minimal intrusive soft and flexible shear force sensor for use on the finger tips that does not impede fingertip touch sensation using FDM 3D-printing technologies?

\section{SoftPro}

The outcomes of this research contributed to the EU SoftPro project, a collaboration between 14 partners from both industry and academia with technical or clinical expertises, which goal was to "study and design soft synergy-based robotics technologies to develop new prostheses, exoskeletons and assistive devices for upper limb rehabilitation, which will greatly enhance the efficiency and accessibility to a greater number of user" [37].

The main goal of this thesis, the development of a distributed sensing system that integrates EMG, kinematic and kinetic data using soft and flexible sensors, is established in collaboration with clinical SoftPro partners. With the aim to develop new methods and sensing technologies to improve the assessment of upper limb motor function of stroke patients.

\subsection{Thesis outline}

This thesis consists of two main parts. The first part is presented in Chapter 2 and concerns a pilot study with the iHAMS system, where 10 chronic stroke subjects performed the arm-related tasks from the Fugl-Meyer Assessment protocol (FMA-UE) with their affected and non-affected side. The goal of this study was to assess the capabilities the iHAMS system in evaluating motor function of the upper limb of stroke patients and in comparing the pathological with non-pathological movement pattern.

The second part of this thesis focusses on the development of 3D-printed sensing structures. Chapter 3 introduces the use of extrusion based 3D-printing for the fabrication of sensing structures and provides an overview of the available printing technologies, materials and properties, sensing technologies, intrinsic limitations and applications of 3D-Printed sensors. Chapter 4 describes the development of 3D-printed EMG sensing structures and compares the performance of 3D-printed electrodes to conventional electrodes. Furthermore, a 3D-printed multi-electrode band for use in real-time gesture detection is demonstrated and 
analysed. Chapter 5 discusses the development and characterization of a preliminary version of a soft 3D-printed piezoresistive shear and normal force sensor for fingertip interaction sensing. The characterization is performed using a finite element model and mechanical measurement setup. An improvement of this sensor design is shown in Chapter 6. The Finger sensor is evaluated on human fingertips using an instrumented object as reference.

Chapter 7 presents the general discussion about the work presented in this thesis and the general conclusions are given in Chapter 8. 


\title{
Chapter 2
}

\section{Quantifying pathological synergies in the up- per extremity of stroke subjects with the use of IMUs}

\begin{abstract}
Stroke is one of the main causes of disability in the world, causing loss of motor function on mainly one side of the body. A proper assessment of motor function is required to help to direct and evaluate therapy. Assessment is currently performed by therapists using observer-based standardized clinical assessment protocols. Sensor-based technologies can be used to objectively quantify the presence and severity of motor impairments in stroke patients. In this work, a minimally obstructive distributed inertial sensing system, intended to measure kinematics of the upper extremity, was developed and tested in a pilot study, where 10 chronic stroke subjects performed the arm-related tasks from the Fugl-Meyer Assessment protocol with the affected and non-affected side. The pilot study showed that the developed distributed measurement system was adequately sensitive to show significant differences in stroke subjects' arm postures between the affected and non-affected side. The presence of pathological synergies can be analysed using the measured joint angles of the upper limb segments, that describe the movement patterns of the subject. Features measured by the system vary from the assessed FMA-UE sub-score showing its potential to provide more detailed clinical information.
\end{abstract}

This chapter is published as: M. Bhagubai*, G. Wolterink*, A. Schwarz, J. Held, B.J. Van Beijnum, P. Veltink Quantifying Pathological Synergies in the Upper Extremity of Stroke Subjects With the Use of Inertial Measurement Units: A Pilot Study, IEEE journal of translational engineering in health and medicine, 2020, *Shared first authorship 


\subsection{Introduction}

Stroke is the third most common cause of disability in the world [1]. Approximately 1.1 million people in Europe [38] and eight hundred thousand people in the United States of America [39] suffer from stroke each year. Up to $50 \%$ of stroke survivors become chronically disabled [40], and experience impairments related to upper limb motor function [4, 41], such as muscle weakness or paresis, spasticity and decreased inter-joint coordination [42].

The normal muscle co-activation patterns exist in a stable spatiotemporal way across different muscles, and work in the sense of performing complex functional movements. The dependent pattern of muscle recruitment and activation is known as motor-synergy. In stroke, the damaged brain cells cause an interruption of the neural pathways. When the cortical cells reorganize, alternative descending pathways emerge [43]. The rearrangement of the descending motor neurons may result in an inevitable joint excitation or inhibition of different muscles. The abnormal co-activation muscle patterns are known as pathological synergies, and are associated with a reduced number of degrees of freedom of the motor control [44]. The pathological synergistic movement in stroke patients have been described in the past by Twitchell [45] and Brunnstrom [46]. In the process of motor function recovery, voluntary movement is characterized by two main muscle-coupling-patterns: the flexor and the extensor synergies. In the flexor synergy, an attempt of movement results in a coupled abduction and external rotation of the shoulder, flexion of the elbow, wrist and fingers, and forearm supination. Similarly, the extensor synergy is characterized by a coupled adduction and internal rotation of the shoulder, elbow extension, wrist and finger flexion, and forearm pronation [44]. These synergy patterns were objectively characterized by measuring the joint torques produced in the upper arm of patients by Dewald et al. [47]. Furthermore, these impairments create a learned bad use of the affected extremity, where compensation strategies are adopted by stroke survivors to increase success in completion of tasks [48, 42].

The importance of assessing motor function rises with the need of proper rehabilitation methods. Motor outcomes due to stroke, and its extent, differ between patients. A subject-specific rehabilitation protocol is needed, and frequent assessment provides better adaptation to the patient's progress [49]. One of the most used assessment scales in the clinic to evaluate motor function is the Fugl-Meyer Assessment (FMA) [50]. The FMA was developed with the foundations of the motor recovery stages described by Twitchell and Brunnstrom [51]. Although it has been used as a gold standard method of upper extremity motor function assessment, it is an ordinal scale with insufficient sensitivity and 
suffers from a ceiling effect when evaluating patients with mild impairment [52]. Despite the high values for reliability and validity, the assessment procedure and scoring requirements have to be well defined in order to correctly assess the patient and avoid subjective variation in the score depending on the assessor.

Recently, kinematic measurements have been used to objectively quantify motor function in the upper extremity of stroke patients. Kinematic assessment can provide several metrics and features that allow an objective evaluation of both motor function and performance $[6,53]$. A wide variety of kinematic measurement systems are used. Optical motion capture systems are considered the gold standard system to measure body kinematics [54, 55, 56]. However, the high cost and the need of a customized laboratory with fixed cameras are the main limitations. Robotic systems with motion capture capabilities such as exoskeletons and end-effectors in combination with arm weight support to control shoulder abduction loading, have also been used [57, 58, 59, 12, 13]. These robotic systems obstruct and have influence on the movement, reducing degrees of freedom in some cases. A widely used kinematic type of sensors are inertial measurement units (IMU). These sensors are low-cost and portable, and do not need specialized laboratories to perform the measurements. The majority of the studies that use wearable IMUs evaluate the relation between daily living tasks and clinical assessment scales, focusing on performance evaluation $[6,53,60,61]$. Objective assessment of the actual FMA upper extremity subscale (FMA-UE) has been done before with accelerometers and gyroscopes [62], but the extracted features did not take in account the sensor's orientation estimate and measurements of joint angles of the different limb segments. The purpose of the distributed sensing system is also to aid clinicians in their evaluation. An objective measurement of the arm joint angles is expected to be easily translatable to the clinic and understood by the therapist.

In this pilot study, a custom upper limb IMU system was developed and used to measure kinematics from, the sternum to the finger tips, of stroke patients while performing items from the FMA-UE. The goal of this study is to assess the capability of a new IMU system in evaluating motor function of the upper limb of stroke patients and comparing the pathological with non-pathological movement patterns. It is hypothesized that the kinematic features measured with the distributed IMU system can objectively distinguish the arm posture of the affected and the non-affected arms of stroke patients when the clinical evaluation tasks are performed. Based on knowledge of the pathological muscle coupling after stroke, it is expected that patients have an increased difficulty in reaching the desired target arm posture during the FMA-UE, when performed with the affected arm. The characteristics of the flexor and extensor synergies, 
such as stronger coupling between the shoulder, elbow and wrist, are also expected to be present on the affected arm's movement profile. Another aim of this study is to evaluate if the system can distinguish more affected from less affected patients based on the FMA-UE score given by the therapist.

\subsection{Methods}

The methods adopted in this work align towards a detailed analysis of the upper limb's movement of a patient. This is accomplished by using a measuring system composed of an increased number of sensors designed to provide a full reconstruction of the upper limb segments whilst being minimally obstructive and portable. The kinematic analysis is of relatively short lasting movements only using inertial sensors, not applying magnetometers due to the sensitivity to disturbances.

\subsubsection{Measurement System}

The used distributed experimental sensing system is composed of eight IMUs (See Figure 2.1). It was based on the previous developed system by Kortier et al. [63]. The system consists of multiple small rigid printed circuit boards (PCB) that are interconnected by flexible cabling, where each rigid section contains a pair of triaxial gyroscopes and accelerometers (ST LSM330DLC). The system is divided into an arm and a hand substring. The arm string consists of four IMUs that are mounted on and measure the kinematics of the sternum, shoulder, upper arm and lower arm. The hand string has IMUs that are mounted on the dorsal side of the hand, thumb, index and middle fingers. A microcontroller (Atmel XMEGA) is responsible for data collection and for the USB interface to the computer. The data is collected at sampling frequency of $200 \mathrm{~Hz}$ for the gyroscopes and $100 \mathrm{~Hz}$ for the accelerometers.

The IMUs are covered by a 3D printed Thermoplastic polyurethane (TPU) housing and were mounted to the respective body segment using 3D printed flexible mounting straps. The shoulder and sternum IMU housings were fixed using medical tape. The sternum IMU was placed at the centre of the chest; the shoulder sensor was placed between the superior border of the scapula and the clavicle, near the acromioclavicular joint; the upper arm IMU was placed on the lateral side of the upper arm, near the elbow; the lower arm sensor was placed on the dorsal side of the forearm close to the wrist; the hand IMU was placed on the dorsal side of the hand, roughly in the centre; the finger sensors were placed in the distal inter phalanges of the thumb, index and middle finger. 


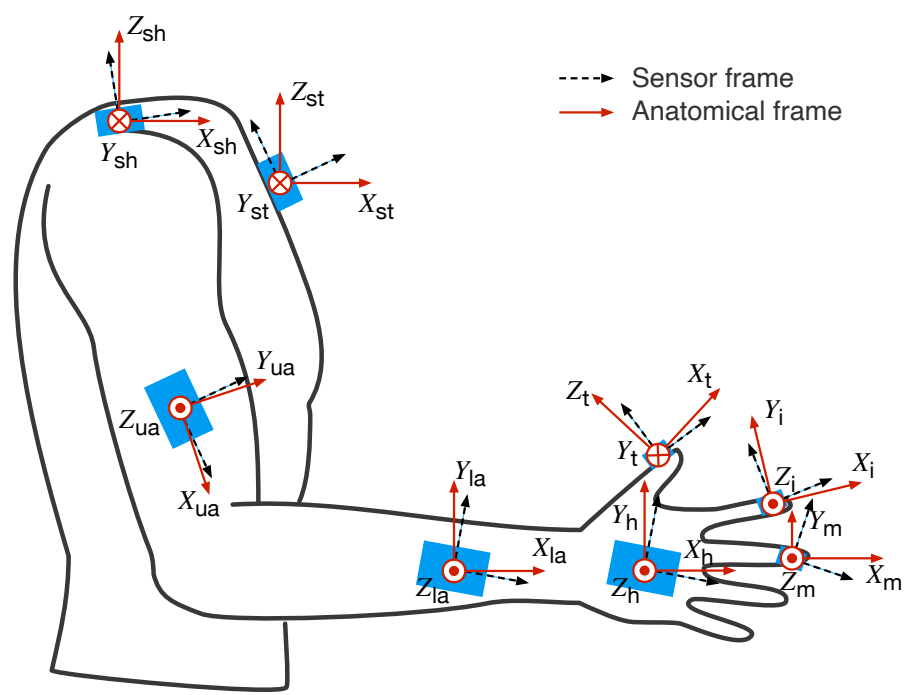

Figure 2.1: Sensor's frames and correspondent anatomical frames of the eight different IMUs of the measurement system placed on the right arm.

\subsubsection{Kinematic Reconstruction}

Since the system measures accelerations and angular velocities, several steps are needed to estimate the orientation of each sensor, and therefore, each body segment.

\section{Sensor-to-segment calibration}

First, a sensor-to-segment calibration was conducted in order to find the orientation of the sensor with respect to the corresponding body segment. The sensor's and the anatomical frames defined are shown in Figure 2.1. The coordinate systems of the sternum, arm and hand are arbitrarily defined in a way that the $\mathrm{x}$-axis of the anatomical frame is in the longitudinal direction of the segment. The alignment of the sensors to the respective limb segments is based on the acquisition of data while the subject is in defined static postures or during dynamic movements. The principle is that measurements of the gravity vector with the accelerometer when the limb segment is in a defined posture represents an axis of the anatomical frame of the respective limb; likewise, measurements of the sensor's angular velocity during a defined dynamic movement also represent an axis of the anatomical frame of one limb segment. This protocol was based on the work of Luinge et al. [64] and Ricci et al. [65]. The description of each posture/movement and the anatomical axis that are defined with each 
Table 2.1: Anatomical axes of the left and right arms defined by each item of the sensor-to-segment calibration protocol.

\begin{tabular}{|c|c|c|c|}
\hline & $\begin{array}{c}\text { Calibration } \\
\text { Position/Movement }\end{array}$ & $\begin{array}{c}\text { Anatomical } \\
\text { Axis Left Arm }\end{array}$ & $\begin{array}{c}\text { Anatomical } \\
\text { Axis Right Arm }\end{array}$ \\
\hline 1 & Static hand flat on a table & $\overrightarrow{z_{h}}, \overrightarrow{z_{i}}$ & $\begin{array}{c}\overrightarrow{z_{h}}, \overrightarrow{z_{i}} \\
\overrightarrow{z_{m}}\end{array}$ \\
\hline 2 & $\begin{array}{l}\text { Static hand sideways, } \\
\text { with elbow flexed } 90^{\circ}\end{array}$ & $\begin{array}{l}-\overrightarrow{y_{h}},-\overrightarrow{y_{i}} \\
-\overrightarrow{y_{m}}\end{array}$ & $\begin{array}{c}\overrightarrow{y_{h}}, \overrightarrow{y_{i}} \\
\overrightarrow{y_{m}}\end{array}$ \\
\hline 3 & Static thumb flat on a table & $\overrightarrow{z_{t}}$ & $\overrightarrow{z_{t}}$ \\
\hline 4 & $\begin{array}{l}\text { Static thumb sideways, } \\
\text { with hand pronated }\end{array}$ & $\overrightarrow{y_{t}}$ & $-\overrightarrow{y_{t}}$ \\
\hline 5 & $\begin{array}{l}\text { Static forearm and hand } \\
\text { palm faced down }\end{array}$ & $\overrightarrow{z_{l a}}$ & $\overrightarrow{z_{l a}}$ \\
\hline 6 & $\begin{array}{l}\text { Wrist pronation, starting } \\
\text { form supinated position }\end{array}$ & $\overrightarrow{x_{l a}}$ & $-\overrightarrow{l_{l a}}$ \\
\hline 7 & $\begin{array}{l}\text { Static shoulder adducted, } \\
\text { with elbow flexed }\end{array}$ & $-x_{u a}$ & $-\overrightarrow{x_{u a}}$ \\
\hline 8 & $\begin{array}{l}\text { Static shoulder abducted } \\
90^{\circ} \text { with elbow flexed }\end{array}$ & $z_{u a}$ & $z_{u a}$ \\
\hline 9 & $\begin{array}{l}\text { Static straight neutral pose, } \\
\text { arm along the body }\end{array}$ & $\overrightarrow{z_{s h}}, \overrightarrow{z_{s t}}$ & $\overrightarrow{z_{s h}}, \overrightarrow{z_{s t}}$ \\
\hline 10 & $\begin{array}{l}\text { Hip flexion (arm moving } \\
\text { along the upper body) }\end{array}$ & $\overrightarrow{y_{s h}}, \overrightarrow{y_{s t}}$ & $-\overrightarrow{y_{s h}},-\overrightarrow{y_{s t}}$ \\
\hline
\end{tabular}

particular calibration movement can be found in Table 2.1. For each sensor, two different axes were measured with the sensors (depending on the segment, either by the accelerometer or the gyroscope), and the third axis was calculated using the cross-product of the previous two axes. Subsequently, one of the first two axes was redefined to ensure orthonormality in the coordinate system.

A median filter was applied to the accelerometer data during the static positions or to the gyroscope data during the dynamic movements in order to get the anatomical frame relative to the sensor's frame:

$$
v_{\text {seg }}=\frac{\operatorname{median}\left(\overrightarrow{a_{s}}\right)}{\left\|\operatorname{median}\left(\overrightarrow{a_{s}}\right)\right\|} \quad \text { or } \quad \overrightarrow{v_{s e g}}=\frac{\operatorname{median}\left(\overrightarrow{\omega_{s}}\right)}{\left\|\operatorname{median}\left(\overrightarrow{\omega_{s}}\right)\right\|}
$$

Where $\overrightarrow{s e g}$ is the anatomical axis relative to the sensor $s$, and $\overrightarrow{a_{s}}$ and $\overrightarrow{\omega_{s}}$ are the accelerometer and gyroscope measurements in the respective calibration static position or dynamic movement respectively. The orientation of the segments' coordinate frame relative to the sensor's coordinate frame is given by a 
rotation matrix that contains three vectors that correspond to the anatomical axes of the segment, expressed in the sensor coordinate system:

$$
{ }^{S} \mathrm{R}_{S e g}=\left[\begin{array}{lll}
x_{\text {seg }} & y_{\text {seg }} & z_{\text {seg }}
\end{array}\right]
$$

\section{Global frame definition}

In order to relate the orientations of the segments to each other, needed for the kinematic reconstruction of the upper extremity, a common global frame needs to be defined. For this definition, the last two movements (static neutral pose and hip flexion) of the sensor-to-segment calibration protocol were used [66]. The static neutral pose, with the arm stretched along the body and the fingers extended, is used to define the common vertical axis by measuring the gravity vector in all sensors. The hip flexion movement is performed with the arms stretched and accompanying the trunk movement. The measured angular velocity is used to define the horizontal axis of the global frame.

\section{Orientation estimation}

With the sensor-to-segment alignment and the common global frame for every IMU, it is possible to reconstruct the movement of the chest, arm and hand by starting in the static neutral pose. Orientation estimation is usually done by integrating the angular velocity over time to get the angular change of the sensor. However, this method causes integration drift due to noise and offsets in the gyroscope measurements. Therefore, sensor fusion algorithms were used to correct both the inclination and heading of the sensor. In this study, a Madgwick filter [67] was used to compensate the IMU inclination errors due to integration drift, by fusing data from the gyroscopes and accelerometers. Zeroangular velocity updates are used to reduce errors in the orientation estimation. This error correction technique was based on the work of Kirking et al. [68]: if the norm of the angular velocity is below $3^{\circ} / \mathrm{s}$, the sensor is considered to be static. By using a linear interpolation between two periods where the sensor is static, the effects of the drift and offset in the gyroscope measure were eliminated during the movement, reducing the integration drift when estimating the sensor's orientation.

The orientation estimation of each sensor $S$ is given in quaternion form and converted to a rotation matrix [69]. Finally, the body segment's orientation relative to the global frame is calculated by aligning the sensor's frame to the anatomical frame using the sensor-to-segment calibration parameters.

$$
{ }^{G} \mathrm{R}_{S e g}(t)={ }^{G} \mathrm{R}_{S}(t){ }^{S} \mathrm{R}_{S e g}
$$


Where ${ }^{G} \mathrm{R}_{\text {Seg }}(t)$ is the orientation of the limb segment at time $t$ relative to the global frame.

\section{Joint angle extraction}

The arm joint angles can be represented as the angle between the anatomical axis aligned with the respective limb segments. The anatomical frames seen from the global frame are represented by the collumns of the rotation matrices calculated in the orientation estimation.

For the elbow, wrist and fingers flexion/extension angles, the angle was calculated between the axes of the two adjacent limb segments. The longitudinal axis of the more distal segment of the joint was first projected to the flexion/extension plane, with the normal vector corresponding to the axis in the medio-lateral direction, of the more proximal segment of the joint. This excludes measuring ulnar or radial deviations or lateral movements of the fingers that do not correspond to flexion or extension of the segment. The shoulder flexion/extension and abduction/adduction was calculated by relating the upper arm frame to the sternum frame. For the flexion angle, the axis aligned with the upper arm is projected onto the body's sagittal plane, which corresponds to the $x z$-plane of the sternum's frame. The abduction angle is calculated by projecting the same upper arm axis onto the frontal plane, which is defined as the $z y$-plane of the sternum's frame. The projected vector $\overrightarrow{v^{p}}$ is given by:

$$
\overrightarrow{v^{p}}=\vec{v}-\frac{\vec{v} \cdot \vec{n}}{\left\|\vec{n}^{2}\right\|} \vec{n}
$$

Where $\vec{v}$ is the axis of the segment's frame and $\vec{n}$ is the plane normal vector. The angle $\theta$ between two vectors $\overrightarrow{v_{1}}$ and $\overrightarrow{\left(v_{2}\right)}$ is given by:

$$
\theta=\operatorname{atan} 2\left(\frac{\left\|\overrightarrow{v_{1}} \times \overrightarrow{v_{2}}\right\|}{\overrightarrow{v_{1}} \cdot \overrightarrow{v_{2}}}\right)
$$

Where atan 2 is the four-quadrant inverse tangent, outputting angles between $-\pi$ and $\pi$. Details on the individual joint angles calculation can be found in appendix 2.5 .

\subsubsection{Experimental Design}

\section{Participants}

Ten moderately affected (FMA-UE between 34 and 54) chronic stroke survivors, recruited from the University Hospital Zurich, Switzerland were included in this study. All participants suffered from a unilateral lesion resulting from either an ischemic or a hemorrhagic stroke. The participants included in this study were 
Table 2.2: Demographic and clinical characteristics of the participants $(\mathrm{N}=10)$

\begin{tabular}{ll}
\hline Characteristic & Value \\
\hline Sex (male/female) & $6 / 4$ \\
Age median (IQR) in years & $61.5(57.5-70.0)$ \\
BMI median (IQR) & $26.5(23.7-29.2)$ \\
Arm length median (IQR) in cm & $68.5(62.6-76.6)$ \\
Dominant hand (left/right) & $0 / 10$ \\
Time since stroke median (IQR) in months & $31.5(20.3-63.8)$ \\
Affected side (left/right) & $5 / 5$ \\
FMA-UE median (IQR) / max & $33.5(32.3-34.8) / 66$ \\
$\quad$ Arm subscore / max & $22.0(22.0-24.8) / 36$ \\
Wrist subscore / max & $6.0(5.5-6.8) / 10$ \\
Hand subscore / max & $9.5(6.8-11.0) / 14$ \\
Coordination subscore / max & $2.5(2.0-3.8) / 6$ \\
\hline
\end{tabular}

required to be at least 18 years old and to have a diagnoses of stroke in the chronic stage ( $>6$ months). All participants had to have stroke-associated impairments of the upper limb (FMA-UE lower than 60) and be at least partially able to move the arm against gravity and to perform finger movements for basic grasp function. Subjects were excluded if they had pre-existing impairments of the upper limb e.g. orthopaedic impairments, severely increased muscle tone and sensory deficits. Participants were also excluded if severe communication or cognitive deficits cause inability to follow the procedures and give informed consent, or if there were contraindications on ethical ground. The FMA-UE test was performed before the start of the protocol to characterize the motor impairment level. Detailed demographic characteristics are presented in Table 2.2.

All participants gave written informed consent in accordance with the declaration of Helsinki. The cantonal ethics in Zurich approved the experimental protocol prior to start of the study (Req-2019-00417).

\section{Experimental protocol}

Before placing the distributed sensing system, the participants were assessed using the full FMA-UE test on the affected and non-affected side by a physiotherapist. The experimental protocol was performed on both limbs separately, starting with the non-affected limb. After donning of the system, the sensor-tosegment calibration procedure was performed. The therapist helped the participant maintaining the static postures and performing the dynamic movements. Each posture was measured for at least five seconds. The protocol with the 
system in place consisted of performing four items from the FMA-UE test to examine movements within and out of the pathological synergies. Each item consist of one or more tasks that are scored on a 0 to 2 scale $(0=$ can not perform, 1 = performs partially, $2=$ performs fully) by a physiotherapist [70]:

1. FMA-UE item A2: movement within the synergies, where the subject is asked to raise the hand from the contralateral knee to the ipsilateral ear from extensor synergy (shoulder adduction/ internal rotation, elbow extension, forearm pronation) to flexor synergy (shoulder abduction/ external rotation, elbow flexion, forearm supination). The movement is scored in a 0 to 2 scale based on the performance of the shoulder retraction, elevation, abduction of $90^{\circ}$ and external rotation and on elbow flexion and forearm supination ( $\max 12$ points).

2. FMA-UE item A3: movement mixing the flexor and extensor synergies, where the subject is instructed to flex the shoulder $90^{\circ}$ and maintain the elbow fully extended $\left(0^{\circ}\right)$. The maximum score of 2 points is achieved when there is no shoulder abduction or elbow flexion, immediate abduction or elbow flexion results in 0 points, abduction or elbow flexion during movement results in 1 point,

3. FMA-UE item A4: movement out of the pathological synergies, where the subject is asked to abduct the shoulder $90^{\circ}$, keep the elbow fully extended $\left(0^{\circ}\right)$ and the forearm pronated. The maximum score of 2 points is achieved this the subject performs this movement flawless. Immediate supination or elbow flexion results in a score of 0 points, during movement in 1 point.

4. FMA-UE item $B$ : intended to evaluate the range of wrist flexion and extension. The subject is asked to flex the shoulder (at least $70^{\circ}$ ) and perform wrist flexion and extension movements while keeping the elbow and fingers extended $\left(0^{\circ}\right)$. A smooth full active range of motion results in maximum score of 2 points, no volitionally movement in 0 points and a limited range of motion in 1 point.

The subjects were seated and performed three repetitions of each item. This procedure is repeated for the affected arm.

\subsubsection{Data Analysis}

\section{Feature extraction}

To characterize the subjects' performance, different features were extracted from measurements. An example of the joint angle estimation for one of the tasks 


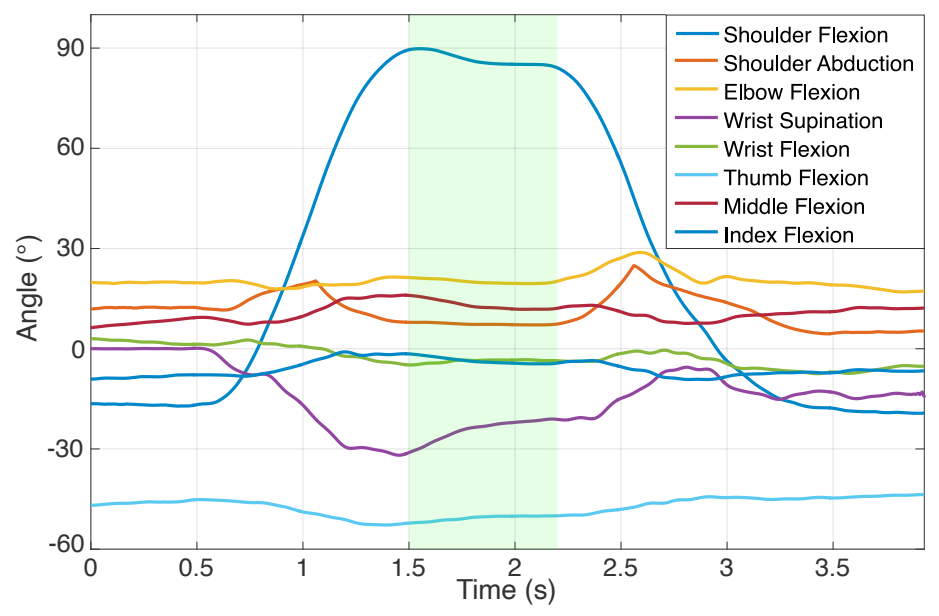

Figure 2.2: Arm joint angles measured in one participant during task 2 of the experimental protocol (item A3). The green shaded area indicates the time interval where the arm is in the target position.

can be seen in Figure 2.2. The figure represents the upper limbs joint angles of a non-affected arm. A positive angle indicates flexion or abduction of a joint, and a negative angle represent extension or adduction. The static neutral pose (with the elbow and fingers extended and pointing down) is considered to be the zero for the joint angles. The features used to evaluate the subjects performance are the joint angles measured in the target position of each task (green shaded area in Figure 2.2).

\section{Statistical analysis}

The participants were divided into two different main groups with different levels of impairment (group 1 and group 2). The groups were created based on the FMA-UE arm subscore. The more affected group (group 1) included subjects with a score of 22 or less $(N=6$, FMA-UE arm subcore median and interquartile range (IQR): $22(22-22)$ ). The less affected group (group 2) was composed by the remaining participants $(N=4$, FMA-UE arm subscore median and IQR: $25.5(24.5-28.5))$. Within each group, the data from the affected (AF) and non-affected (NAF) arms was separated, creating in total four different groups for the statistical analysis (NAF-1 - non-affected arm of group 1; AF-1 - affected arm of group 1; NAF-2 - non-affected arm of group 2; and AF-2 - affected arm of group 2).

Two different statistical tests were applied. First, the Wilcoxon signed rank 
test was used to analyse differences between the extracted features within the same impairment level group (differences between NAF-1 and AF-1 and between NAF-2 and AF-2). Second, the Mann-Whitney U test was used to check differences between the measurements of groups 1 and 2, of the same arm (differences between NAF-1 and NAF-2 and between AF-1 and AF-2).

Both tests are non-parametric due to the small sample size and the low evidence of normally distributed data. All statistic analysis was done with a significance level of 0.05 .

\subsubsection{Validity tests}

Before the experiments with stroke subjects, the validity of the distributed system and the experimental protocol was tested using a rigid arm model and a healthy subject test. The rigid arm model, consists of a wooden frame composed of a body and two wood parallelepiped pieces, around the size of a human arm. The "upper arm" wooden piece is connected to the body via two eye hooks. The "lower arm" piece is connected to the upper arm via a hinge. It simulates the shoulder joint, allowing for flexion and abduction, and an elbow joint that is able to flex from a fully extended posture (elbow flexion angle of $0^{\circ}$ ). The rigid model test simulated multiple $90^{\circ}$ movements for the shoulder flexion, shoulder abduction, elbow flexion, wrist flexion, extension, pronation and supination. The wrist movements were simulated by attaching the sensor to an additional wooden block and manually moving it in the desired direction. The results of the rigid model test correspond to the angles calculated using the orientation estimation and feature extraction methods. The estimated reconstructed angles of the shoulder flexion $\left(87^{\circ}\right)$, abduction $\left(90^{\circ}\right)$, elbow flexion $\left(90^{\circ}\right)$ and the wrist flexion $\left(88^{\circ}\right)$ and extension $\left(87^{\circ}\right)$ show errors less than $3^{\circ}$ in the target position of the tested $90^{\circ}$ movements. The wrist supination/pronation $\left(102^{\circ} / 99^{\circ}\right)$ tests with the rigid model show an overestimation of the joint angle of around $10^{\circ}$. During the tests, the overall angles of the other joints are significantly small (mean and standard deviation $-1^{\circ} \pm 2$ ). The finger flexion angles show a higher deviation than the other joint angles during the tests $\left(4^{\circ} \pm 3\right)$

The second test involved a healthy subject (Male, Age: 23, BMI: 22.1, Arm Length: $73 \mathrm{~cm}$ ), since healthy individuals have the ability of performing more controlled movements, for the global frame definition, the sensor-to-segment protocol and the actual movements of the protocol. These tests were approved by the University of Twente faculty ethics committee. The measured joint angles in the target position showed some variations in the different repetitions. However, the mean joint angles were close to the instructed angles. In movements 
2 and 3 (shoulder abduction and shoulder flexion), the mean of the measured angles of these joints are $89^{\circ} \pm 1^{\circ}$ and $92^{\circ} \pm 4^{\circ}$, close to the desired $90^{\circ}$ angle. The subjects were instructed to fully extend the elbow in tasks 2,3 and 4 . The measured mean elbow flexion angles are close to $0^{\circ}\left(-2^{\circ} \pm 5^{\circ}\right)$. The negative values can be caused by overextension of the elbow. Other joint angles are consistent throughout the protocol, where the highest standard deviation of all the measured features was $15^{\circ}$, corresponding to the middle finger flexion angle in movement 4 .

\subsection{Results}

All ten stroke subjects were able to complete the experimental protocol. Data from the thumb IMU was discarded for one subject due to malfunctions of the sensor. In one repetition of the fourth item of the protocol, the IMU data had to be excluded due to recording errors. In total, 119 movements were analysed (30 repetitions of tasks 1, 2 and 3 of the protocol and 29 of task 4).

Figure 2.3 shows the joint angles in the target position measured in the four different groups when subjects performed the experimental tasks. The median and the IQR ( $25^{\text {th }}$ and $75^{\text {th }}$ percentiles) of the measured relevant joint angles of the group are shown in the form of boxplots. The presented joint angles allow the visualization of possible pathological synergistic behaviour in the proximal limb segments (shoulder and elbow). The considered relevant joints were based on the scoring criteria of the FMA-UE [52]. The desired target joint angles for each task are marked with dashed lines in the figure. It can be seen that there are significant differences $(P<0.05)$ between the four data groups, mainly at the shoulder and elbow level. Depending on the task, results show that the desired target position is harder to reach when the more affected subjects perform the task with the affected arm (AF-1). The shoulder flexion angle of AF-1 is significantly lower in task 2 and task 4 and the shoulder abduction is significantly lower in task 3 compared to the non-affected arm (NAF-1) and less affected group (AF-2). The elbow is significantly more flexed in the more affected groups when compared to the respective less affected groups (AF-1 vs AF-2 and NAF-1 vs NAF-2).

The relation between the FMA-UE score and the measured features can be seen in Figure 2.4. The shoulder and elbow flexion angles of the AF and NAF arms of all subjects on the target posture of task 2 (shoulder flexion movement) are plotted against the FMA-UE score of the participants. The figure presents the mean of the joint angles measured on the three repetitions of each subject. Similarly to what is observed in Figure 2.3, more affected subjects have a smaller 

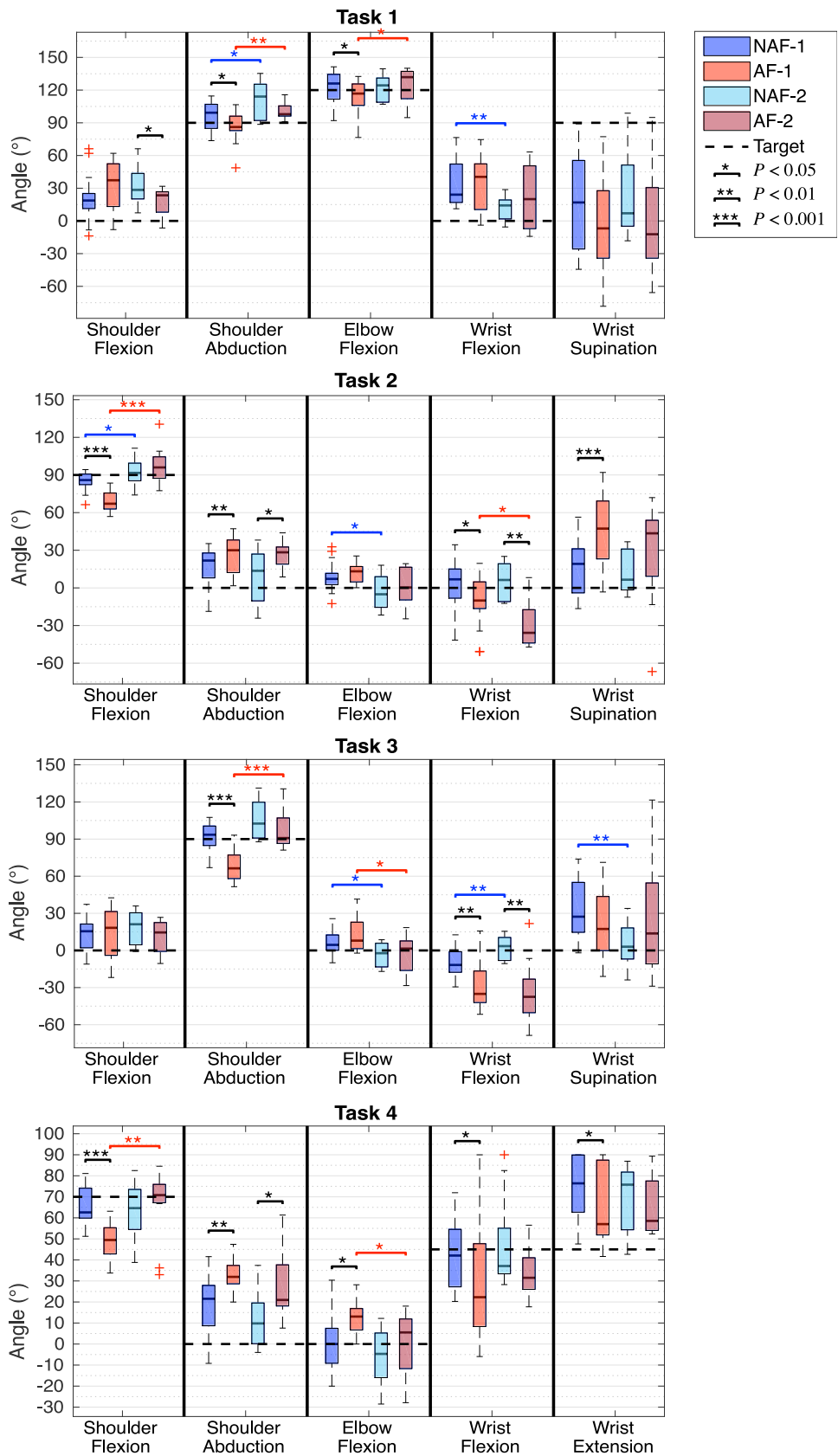

Figure 2.3: Boxplots (median and IQR) of the target angles for the most significant joints evaluated in the four tasks of the protocol in the four groups. The black dashed lines represent the desired targets for the task. Significant differences between arms of the same group are indicated with a black line. Significant differences between the same arm of different groups are indicated with a blue (NAF) or red (AF) lines. $* P<0.05 ; * * P<0.01 ; * * * P<0.001$. 

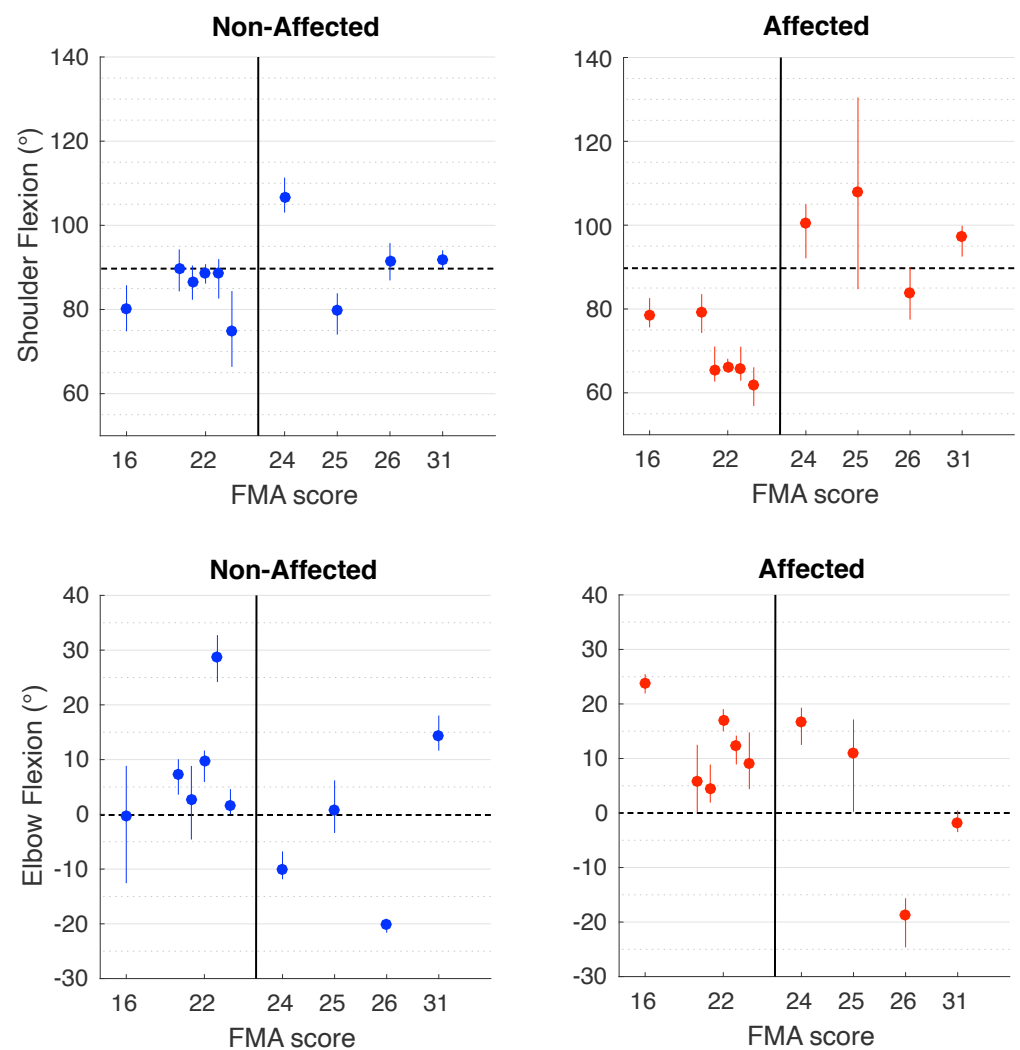

Figure 2.4: Relation between the shoulder and elbow flexion of the AF (red) and NAF (blue) arms and the FMA-UE arm subscore during task 2 (FMA-UE item A3) of the protocol. The black dashed lines represent the desired targets for the task.

shoulder flexion angle and a higher elbow flexion. The most affected subject (with FMA-UE of 16) appears to have a higher shoulder flexion angle than the subjects with an FMA-UE score of 22. In addition, the five participants with a score of 22 have different arm postures.

\subsection{Discussion}

Measuring kinematics during clinical motor function assessment scales has not been performed extensively in the past. The majority of studies use kinematic measures in activities of daily living or while performing functional tasks, such as reach-to-grasp tasks $[71,54,48,72]$. Some studies used inertial measurement 
units while performing simple movements that include flexion/extension, abduction and supination of limb segments $[62,72]$. In this work, a new distributed sensing system was developed and tested in an upper limb model, healthy and stroke subjects. The kinematic analysis with the developed distributed IMU measurement system is a minimally obstructive technology that uses IMUs for quantification of arm function. By performing the arm-related tasks of the clinical scale, the presence of the described pathological synergies can be analysed via features that describe the movement patterns of the patients. The results presented in Figure 2.3 and the results from the statistical analysis show significant differences between the postures of the affected and non-affected limbs. However, some might not be clinically relevant.

The measured joint angles during the first task show a similar behaviour in the shoulder and elbow flexion of the AF and NAF arms. The shoulder abduction angle is significantly lower in the more affected group of subjects when compared to the less affected group (a difference of about $20^{\circ}$ ), both in the NAF and AF arms. However, the median is higher than the desired target angle. These findings suggest that when the movement is performed within the flexor synergy (abduction of the shoulder accompanied by elbow flexion and wrist supination and flexion), the kinematic differences might not be related to the presence of the flexor synergy, but to muscle weakness or paresis, causing the inability to abduct the shoulder against gravity. The lower shoulder and elbow range of motion is only noticed between the affected and non-affected arms of the more impaired group, indicating that less affected subjects have indeed fewer effects of paresis. The wrist supination angles are below the desired target angle in all groups, having a very high variance in the results. Effects of pathological coupling are inconclusive in the wrist joint.

The second task is intended to mix the flexion and extension synergies. The results show that the shoulder flexion angle is significantly lower in the affected side of the more impaired group of subjects. The measured joint angles indicate that there is a higher elbow flexion angle in the AF side of both groups of subjects when compared to the NAF of the respective group. This behaviour becomes clinically relevant due to the high median difference between groups, the $\mathrm{AF}$ arm of the most affected group shows a shoulder flexion and an elbow flexion median angle $20^{\circ}$ and $10^{\circ}$ lower than the NAF arm respectively. The subjects were clearly instructed to perform the movement with their elbow completely extended. The cause of a lower shoulder flexion can be related to weakness or a compensation strategy to avoid elbow flexion. It is possible that they prioritize the elbow angle and not the shoulder flexion. The shoulder abduction angle is higher in the affected arm of both groups of subjects, which is a manifestation 
of the flexor synergy, where the shoulder flexion is accompanied by involuntary abduction. Finally, the wrist supination angle only shows to be significantly higher in the affected arm of the less impaired group when compared to the non-affected arm of the same group, which is also a behaviour present in the described pathological flexor synergy [43].

The third task (shoulder abduction) shows a significant difference between the affected and non-affected limbs. When the task is done with the affected arm of the more affected group, the shoulder is not sufficiently abducted in order to reach the desired target angle, only being able to reach around $20^{\circ}$ below. The pathological synergy behaviour is, however, not clearly visible in this task since the elbow flexion angle does not show significant differences between arms of the same group, and the median value difference between arms of the different groups of subjects is smaller than $10^{\circ}$. Similarly to the previous task, it is possible that subjects prioritize the extension of the elbow and abduct less the shoulder.

The fourth task, intended to evaluate wrist function, shows expected results, the wrist flexion and extension is significantly higher in the non-affected arm when compared to the affected limb of the more impaired group. The shoulder flexion angle in this movement is not well-defined for the subjects. They are instructed to flex the shoulder $70^{\circ}$, which is not easy for the subject to determine where it is. Even so, it appears that the shoulder flexion angle is significantly lower in the AF arm of the more affected group (approximately $20^{\circ}$ smaller than the target). The elbow flexion is significantly higher in the affected limb, as well as the shoulder abduction, which follows the observations of pathological synergies. Both these joint angles appear to have a median $15^{\circ}$ higher in the AF1 group when compared to the NAF-1 and NAF-2. It has been observed in the past that wrist and finger motor function is mostly the last to recover [45, 73].

The statistical tests done between the upper extremities of the more and less affected groups (group 1 and 2 respectively) suggest that the variation of the level of impairment is related to the features presented in this study, and also to the presence of a more severe pathological synergistic behaviour. A significant decrease in the shoulder flexion or abduction and elbow flexion of group 1 to group 2 can be noticed in tasks 2 and 3, showing signs of joint coupling. The more impaired group of subjects shows a significantly lower shoulder flexion in the affected and non-affected arms of the more impaired group when compared to the correspondent side of the less impaired group in task 2 (shoulder flexion movement). However, the difference between the affected arm's elbow flexion angle of group 1 and 2 is not significant, where the median difference is smaller than $10^{\circ}$. In task 3 (shoulder abduction movement), the shoulder abduction 
angle is significantly smaller and the elbow flexion is higher in the more affected group. The elbow flexion difference is seen between both the affected arms and the non-affected arms of the two groups, whereas the shoulder abduction difference is only seen between the affected arms of groups 1 and 2 . This could suggest that the ipsilesional upper extremity is also affected by stroke. This finding is supported by previous research [74], where it was found that the ipsilesional arm also suffers from less severe motor impairments after stroke. Another explanation would be that the subject does not perform the movement well with the non-affected arm due to factors not related to stroke, and only due to age-related weakness or low flexibility in the joints. Nevertheless, the results show that the difference between the shoulder abduction of the AF and NAF arms in the same group of subjects is significant in group 1 but not in group 2, indicating that the less impaired subjects perform the task similarly with both arms, having less severe pathological muscle coupling. The decreased coupling is also seen in the fourth task in the elbow flexion and wrist flexion/extension angles. Less impaired subjects have a lower elbow flexion angle at the affected side when the wrist is flexed or extended. The wrist range of motion, however, does not vary significantly between the arms of the same group. However, care needs to be taken when comparing group 1 and group 2 since the deviation of the participants into the more affected group 1 with a FMA-UE score of 22 or less, is made for illustrative purposes and not an established method.

Several objective kinematic features have been shown to have a strong correlation with clinical scales $[75,76,48,72,62]$. The relation between the measures shown in the present study and the level of impairment, although it follows the theoretical aspects that qualify motor function in stroke patients, does not have a high statistical power due to the low sample size and the small range of impairment severity in the population. Figure 2.4, shows that the results strongly varies among the subjects and only have a limited relation with FMA-UE arm score. E.g. the most affected subject (FMA-UE arm sub score of 16) appears to be able to flex the shoulder $15^{\circ}$ more than less affected subjects (with a score of 22). Despite this, the coupling effect in the affected arm is noticeable and relates better with the clinical score, where the most affected subject shows the highest elbow flexion angle and the less affected show smaller angles. Furthermore, the inter-individual differences shown in Figure 2.4 are large and the variability does not clearly relate to the FMA-UE score. This might be caused by the more fine grain scale of the IMU system compared to the FMA-UE, in which a score of 1 covers a wide range between 0 (can not perform) and 2 (performs fully). This may mean that the sensing system provides complementary info to the more general FMA-UE clinical score. However, clinicians still need to assess whether 
this complementary info is of clinically relevant value.

In this study, the only features used in the movement analysis consisted of joint angles. Several other types of features related to the movement profile can indicate the presence of motor function deficits, like movement smoothness and velocity profiles $[75,72,62,77]$. These features were not explored, however, the data acquired allows for additional analysis. The measurement of the joint angles at different time points of the task can be of interest as well. In the tasks performed in this experiment, the FMA-UE score is based on the range of motion of the joints and also at the moment where the coupling of the shoulder and elbow starts. This moment is hard to be visually identified in less impaired stroke patients, as well as the smoothness and the velocity profiles. The sensors used in the distributed measurement system developed for this study have the ability to measure these features. Another approach is to study the presence of pathological synergies as a response to shoulder abduction loading on the range of monition and the hand and finger dexterity as described by Ellis et al. [13]. This has been studied, using the same system, by handling objects with certain weights and thus functionally applying shoulder abduction loading [19].

Although, the subjects did not report any experience of movement limitations caused by the system, donning and calibrating takes a considerable amount of time. Further studies could lead to a set of clinical relevant features, that could be measured using a reduced set of optimal placed IMUs decreasing the obtrusiveness of the distributed measurement system by reducing the donning and calibration time.

Although the results of this study followed expected behaviours of stroke patients and the described coupling patterns of the pathological synergies, some limitations were identified. Different sources of errors could have a negative impact in the arm orientation estimation. The quality of the measurements was assumed to be acceptable based on trials performed on a rigid model of the arm and on healthy subjects. From the validity tests with the model, it can be concluded that the system's configuration and processing methods can measure shoulder and elbow joint angles with a maximum deviation from the desired target of $3^{\circ}$. When the experimental protocol is performed by the healthy subject the deviations from these joints are higher (maximum of $5^{\circ}$ ). The differences between the real and measured joint angles can be explained by several factors. Firstly, the movement performed is never perfectly the same in different repetitions. Secondly, the sensor-to-segment calibration protocol and the global frame definition may not be perfectly executed, causing deviations between the measured joint angles and the actual arm posture. The therapist helped the stroke subjects maintaining the required postures or performing these dynamic 
movements, however, it was not always possible to perform these movements correctly. Furthermore, the sensor-to-segment calibration relies on an equal body shape for each subject. The effect of this assumption is visible Figure 2.4, where the subject with a score of 26 appears to have a very high negative elbow flexion of $-20^{\circ}$, in both the AF and the NAF arms. To some extent, this could be due to over extension, as known for the subject with a FMA score of 24 which showed $\mathrm{a}-10^{\circ}$ elbow flexion. However, the $-20^{\circ}$ on both the AF and the NAF seems to be caused by errors in the assumptions made during calibration, i.e., when the elbow is not completely extended in the calibration postures a full extension looks like overextension. Task 2 and 4 in Figure 2.3 also indicate that some subjects have a high negative elbow angle on both AF and NAF side. The cause might be that some subjects have more body volume, which affects the anatomical axis measurements during the static postures of the sensor-tosegment calibration protocol. In this particular case, in item 7 of the protocol, the inclination of the upper arm caused by a non-fully adducted posture creates misalignments between the gravity vector measured by the sensor and the anatomical x-axis of the limb segment. More calibration movements would increase the accuracy in the joint angle estimation and the estimated angles would correspond better to reality. Another source of error is the lack of consistency of the initial position for each task. The kinematic reconstruction is based on the orientation of the initial posture, defined by the orientation of the sensors related to the global frame before the task execution. This orientation is used to reset the orientation of the sensors and reduce drifts caused by gyroscope and accelerometer bias, noise and possible external factors that affect the measurements (like temperature and sudden movements). If the subject deviates a lot from the initial posture defined in the beginning of the experiment, the orientation estimation of the task will be prone to errors. To accurately assess the validity of the proposed methods, a reference system should be used, such as optical motion trackers. Nevertheless, the tests done using the rigid arm model and on the healthy subject show that the system and the analysis methods are reliable. Furthermore, the system is capable of visualising the expected synergies. However, the sources of error have to be taken into account and a further investigation should be done to validate the proposed methods. The usage of the distributed IMU system developed in this work can increase the impairment's evaluation quality and eliminate the subjective perspective of the human eye. The complementary use of the system during the FMA-UE test is of interest since it objectively measures the observations done by the therapist during the assessment and provides a detailed analysis of the movement. 


\subsection{Conclusion}

The presented pilot study showed that the developed distributed measurement system is capable of distinguishing movements of the affected and non-affected upper extremities of stroke subjects using inertial sensors. The distributed IMU system can objectively measure kinematic features, such as joint angles, to assess motor function of subjects when they perform tasks of the FMA-UE test. The pilot study on 10 chronic stroke subject showed that the system can identify the presence of pathological muscle couplings and measure features related to the pathological synergies present in the movement of stroke subjects. Also, the severity of the pathological coupling reflected by the arm joint angles is related to the level of impairment of the subjects, where the more affected subjects show the presence of more severe pathological synergistic behaviour and weakness in the shoulder, elbow and wrist joints. The system has the potential to provide more detailed information with respect to the FMA-UE sub-score, since the individual joint angles show no clear relation with the FMA-UE score. Despite the fact that the sensor-to-segment calibration in stroke subject needs to be evaluated in more detail, it is concluded that the distributed measuring system shows high potential as a useful tool for assessing motor function of the upper extremity of stroke subjects.

\section{Appendix - Joint angles calculation}

Shoulder joint angles The shoulder flexion/extension angle is calculated by firstly projecting the x-axis of the upper arm frame $x_{u a}$ onto the $z x$-plane of the sternum, by taking equation 2.4 , with $\vec{v}=\overrightarrow{x_{u} a}$ and $\vec{n}=\overrightarrow{y_{s t}}$. The flexion/extension angle $\theta$ is calculated by taking the angle between $\overrightarrow{x_{u a}}{ }^{p}$ and $-\overrightarrow{z_{s t}}$. If $\theta=0^{\circ}$ means that the shoulder is straight next to the body, pointing down. A positive $\theta$ corresponds to shoulder flexion and a negative $\theta$ to shoulder extension. The shoulder abduction/adduction angle is calculated by projecting $x_{u a}$ to the $z y$-plane of the sternum's frame $\left(\vec{n}=\overrightarrow{x_{s t}}\right)$. A positive angle indicates abduction, a negative angle corresponds to adduction.

Elbow joint angles The elbow flexion/extension angle is taken by projecting $\overrightarrow{x_{l a}}$ onto the $z x$-plane of the upper arm's frame. A positive angle means elbow flexion. If $\theta=0^{\circ}$, it indicates that the elbow is fully extended. Negative values for this joint angle mean that there is elbow over-extension. 
Wrist joint angles The wrist flexion/extension angle is calculated the same way as the elbow, but by projecting the x-axis of the hand's frame $\overrightarrow{x_{h}}$ onto the $z x$ plane of the lower arm's frame. A positive angle indicates wrist flexion and a negative angle wrist extension. If the angle is $0^{\circ}$, it means that the wrist is in the neutral position. Wrist pronation/supination is calculated differently, since there is no plane that accompanies the movement of the hand in order to correctly measure the desired angle. The lower arm pronates and supinates along with the wrist, so comparing its frame to the wrist's frame will not represent the true supination/pronation angle. For this case, the joint angle corresponds to the integration over time of the $x$ component of the gyroscope of the hand IMU. The gyroscope data is first aligned to the segment by multiplying it by the correspondent sensor-to-segment calibration rotation matrix. A positive angle indicates that the wrist is pronated and a negative angle represents a wrist supination. When $\theta=0^{\circ}$, the wrist is in neutral position.

Finger joint angles The index and middle fingers flexion/extension angles are calculated by projecting the finger's x-axis, $\overrightarrow{x_{i}}$ and $\overrightarrow{x_{m}}$, onto the $z x$-plane of the hand's frame. Then, the angle between the x-axis of the hand $\overrightarrow{x_{h}}$ and the projected axis of the fingers ${\overrightarrow{x_{i}}}^{p}$ and ${\overrightarrow{x_{m}}}^{p}$ is calculated. The thumb flexion/extension angle is calculated by projecting $\overrightarrow{x_{t}}$ onto the $x y$-plane of the hand, and calculating the angle between $\overrightarrow{x_{h}}$ and ${\overrightarrow{x_{t}}}^{p}$. A positive angle indicates finger flexion and a negative angle represents extension. 


\title{
Chapter 3
}

\section{Extrusion based 3D printed sensors}

\begin{abstract}
D}$ printing sensors allows for design freedom and customization making it an interesting technique for e.g. the development of integrated sensing structures and biomedical applications. This chapter provides an overview of extrusion-based 3D printing technologies, materials, sensing principles and the applications. Furthermore, the challenges during fabrication, the anisotropy of the printed structures and the use of nonlinear materials in 3D printed sensors will be discussed.
\end{abstract}

Most parts of this chapter are taken almost verbatim from of a collaborative work published as: M. Schouten, G. Wolterink, A. Dijkshoorn, D. Kosmas, S. Stramigioli, G. Krijnen, A review of extrusion-based 3d printing for the fabrication of electro-and biomechanical sensors, IEEE Sensors Journal, 2021

Additionally, the last paragraph of section 3.7 is a summary of the work published as: G. Wolterink, R. Sanders, G. Krijnen, Thin, flexible, capacitive force sensors based on anisotropy in 3D printed structures, 2018 IEEE SENSORS, 1-4, 2018 


\subsection{Introduction}

Weller et al. [78] have identified four aspects of 3D printing that enable it to compete with traditional manufacturing. These aspects are the versatility of the manufacturing method, free customization, free product design complexity and the reduction of assembly. The versatility and customization of the technique make the technique extremely well suitable in for example biomedical and research applications, where often small volumes of custom parts of many types are needed. The free product design complexity and the reduction of assembly introduce a huge opportunity for sensing, increasing the feasibility of large complex networks of sensors. Other authors $[79,80]$ also concluded that additive manufacturing can compete with traditional manufacturing in specific situations. Therefore, it is no surprise that a lot of research on a wide range of $3 \mathrm{D}$ printed sensors $[81,82,83]$ has been done over the last couple of years.

The most basic form of 3D printing consists of extruding fluid material in lines, actually track elements or 'traxels' [30], which solidifies after being deposited. Since this form of $3 \mathrm{D}$ printing is comparatively simple, it is also the most affordable and accessible [84, 85]. Furthermore, the technology can be relatively easily expanded to include multiple materials. For these reasons, this work is limited to extrusion-based 3D printing.

This chapter will give an overview of the available printing technologies, materials, sensing technologies and applications that can be used for extrusionbased 3D printed sensors. Furthermore, the intrinsic limitations of 3D printed sensors, such as the anisotropy of the conductors and nonlinearity of the materials will be discussed.

\subsubsection{Applications of 3D printed sensors}

3D printing technology enables the creation of low-cost and highly personalized structures [86]. Furthermore, the availability of soft and flexible materials make 3D printing an ideal technology for biomedical applications [87, 88, 89, 90, 91, 92]. These soft materials easily follow complex body contours and have proven to be a useful alternative to gold standard electrodes for the use as biopotential electrodes to capture electroencephalography (EEG) [93] and electromyography (EMG) [90] signals. This opens the opportunity to create personalized electrode grids that could, for example, be co-printed with a prosthetic socket. Other applications are found in the development of finger strain sensor gloves to measures finger bending [87], tactile and pulse sensing [94]. 
Another application area is in soft robotics; 3D printing delivers a single fabrication process for soft robots with integrated sensors, actuators, control and power delivery [95]. This is especial beneficial for applications where integration is key for closed-loop control [96]. Current research mainly focuses on single sensors, where piezoresistive and capacitive sensors are used mostly for strain measurements [97, 95]. However, so far integrated 3D printed sensors with actuators for soft robotics are limited in literature [96]. The bulk of the work with traxel-based printing focuses on integrated piezoresistive strain sensors in $3 \mathrm{D}$ printed pneumatic actuators [92, 98, 99, 100]. For pneumatic actuators either the full actuator can be made out of piezoresistive material [92], strain gauges can be embedded by means of printing [98, 101] or including conductors e.g. silver paste [99]. Other work presents the use of conductive TPU as a combined heater and piezoresistive angle sensor in a variable stiffness gripper [102].

The required limited dimension and spatial distribution are still challenges for current 3D printing technologies. Among others, FDM still has limitations in terms of resolution, speed and material compatibility and can only use thermoplastics, which may not be resilient and stretchable enough for use in soft robotics [95]. 3D printing provides new opportunities for development of integrated sensing structures. However, research on this topic has only just started; if the printing resolution can be increased, a higher density of sensors and actuators becomes possible, enabling improved soft robots and novel biomedical applications.

\subsection{Technologies for extrusion based 3D printed sen- sors}

Most sensor designs consist of multiple types of materials. Therefore, the used $3 \mathrm{D}$ printing technology should be capable of handling multiple types of materials, such as conductors, dielectrics, flexible and stiff materials, in order to fabricate $3 \mathrm{D}$ printed parts with integrated sensors.

\subsubsection{Extrusion systems}

Each additional extrusion technique adds a whole new range of materials that can be printed. This section will discuss a non exhaustive list of currently available extrusion techniques. 


\section{FDM}

Fused deposition modeling (FDM) is an additive manufacturing technology where a 3D-object is built up by melting and depositing a thermoplastic material through a nozzle on a building platform where it solidifies. The material is deposited in lines (traxels). To create height, layers of the material are stacked on top of each other [103]. This extrusion method is widely used due to its opensource and low cost nature [81]. The raw materials are in the form of filaments which allow the materials to be easily pushed through and melted by the hot-end by an extruder. FDM offers a wide variety of materials with different properties ranging from stiff to flexible [81, 90]. However, there are limitations on which materials can be printed together due to differences in melting temperatures, shrinkage, chemical composition and wetting behavior.

In general two types of FDM system can be distinguished: the Bowden and the direct-drive setups as shown in Figure 3.1. In the Bowden setup, the extruder is decoupled from the moving hot-end and the filament is transported using a Bowden tube. Since the extruder is decoupled from the moving hot-end, the print head can be smaller and lighter compared to the direct-drive setup. However, in the distance between the extruder and the hot-end, flexible filament is easily compressed and may incur stiction and friction forces on contact with the tube-wall. This leads to inconsistencies in the filament flow, making the Bowden setup less suited for flexible filaments. However, the performance might be improved by using a Bowden tube with tight tolerances [104].

In the direct-drive setup, the extruder is placed on top of the print head, leading to a short straight filament path between the extruder and the hot-end. To increase the performance for flexible filaments even further, the extruder should have a fully constrained path between the drive gear and the hot-end to prevent the filament from escaping from the extruder (see Figure 3.1).

\section{Direct ink writing}

Direct ink writing is a technique where ink or paste is deposited through a needle to create or to add functionality to objects. Direct ink writing can be used to print inks for which the polymerization is catalyzed by heat or light. The solid content in the final object can be higher than for FDM and therefore, materials with properties more similar to that of solid content inside the ink such as metals [105], ceramics [106] or wood [107] can be deposited.

\section{Embedded fibers}

Recently, the opportunity to embed fibers in FDM printing has significantly improved the mechanical performance of FDM printed parts [108]. Furthermore, 

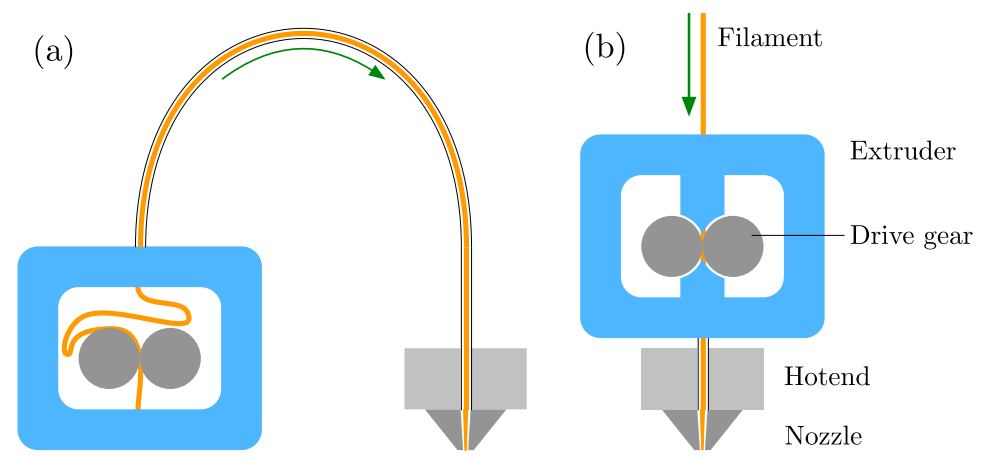

Figure 3.1: The filament can be transported in two possible ways to the hot-end. By a Bowden setup (a), where the extruder is decoupled from the hot-end, and a direct-drive (b), where the extruder is mounted on the hot-end. Fully constraining the filament path (as shown right) prevents tangling of the filament.

this technology allows for fibers or wires to be embedded during fabrication for the use of their electrical properties. Three main methods exist for 3D printing with embedded fibers: fiber placement, fiber co-extrusion and embedding wires after printing.

Fiber placement is a technique in which fibers or wires are laid onto the surface in between the printing of layers. The fiber placement can be done by hand [109], ironed into the material with a separate nozzle [110] or roller [108] during fabrication or even placed by hand after fabrication [111].

Fiber co-extrusion is the technique in which a wire of fibers is extruded together with the molten matrix material. This can be done through the same nozzle $[112,113,114]$ or through a different nozzle directly into the molten material (also called fiber encapsulation) $[115,116]$. Single nozzle printers limit the control over fiber placement, which is the reason most machines have separate nozzles or separate the control of fiber co-extrusion and plastic extrusion.

Embedding the wires after printing can be performed by means of localized ultrasonic or Joule heating $[117,118,119]$. The use of this technique is limited since wires can only be embedded close to the surface and in limited geometries.

Embedding fibers poses several challenges for fabrication. For example, the minimum layer thickness is dictated by the diameter of the used fiber with coextrusion, whereas the diameter combined with the mechanical properties of the fiber limit the bending radius that can be achieved for placement [120]. A major limitation is posed by the layer-wise fabrication of FDM, since fibers are strictly printed in single layers, producing anisotropic properties [110]. Finally, it is difficult with $3 \mathrm{D}$ printing to achieve high fiber volume fractions $(>50 \%)$ 
and to minimize voids between fibers [108].

\subsubsection{Challenges}

In many cases, the fabrication of sensors requires multiple materials. In order to print these materials with the same 3D printer, the printer needs to be capable to switch between the materials. The switch between different materials however, is not trivial, and several challenges exist to prevent issues like contamination and misalignment between the materials.

When using a single nozzle and switching materials, a residue of the material will be left behind in the nozzle. Therefore, when a new material is inserted, it will often be contaminated with the previous material. As a consequence, each time a material change takes place, large amounts of filament have to be purged as means to remove old residue from the filament path, resulting in substantial waste of material and largely increased printing times [121].

Often it is not possible to completely turn off the extrusion of the nozzles instantaneously and as a consequence, some material oozes from the inactive nozzle. Furthermore, if other tools than the active tool-head are moving during printing, they might physically contaminate or deform the already deposited parts.

Adding multiple tool-heads to a printing system can result in a large mass to be accelerated, especially in the case of direct-drive tool-heads. If the printing process requires this mass to be accelerated rapidly, this will impose tough constraints on the mechanics of the printer, increasing the cost of the printer. Therefore, the challenge is to limit the mass that is moving in the printing directions ( $x$ and $y$ ), e.g. by using a Bowden setup (sub-optimal) or moving the bed instead of the nozzles.

Finally, it is often challenging to obtain sufficient repeatability of the system so that the different tools are aligned to each other and stay aligned throughout the printing process.

\subsection{Materials}

The aforementioned extrusion systems can be used to print a wide range of structural materials, ranging from very flexible [122] to very stiff [123]. In order to fabricate 3D printed sensing structures, besides the dielectric material, often both conductive and sensing materials are used. Where the conductive material can double as sensing material or be used for interfacing with the sensing materials. 
Table 3.1: Overview of commercially available conductive materials for FDM printing

\begin{tabular}{llll}
\hline Name & Host & Doping & Volume resistivity $[\Omega \mathrm{cm}]$ \\
\hline Proto-Pasta [132] & PLA & Carbon black & $30(\mathrm{x}, \mathrm{y}) 115(\mathrm{z})$ \\
BlackMagic3D Conductive Graphene [133, 134] & PLA & Graphene and Graphite & 0.6 \\
Multi3D, Electrify [135] & Biodegradable polyester & Copper & 0.006 \\
PI-ETPU 95-250 Carbon Black [136] & TPU & Carbon black & $<300$ \\
EEL [137] & TPU & Carbon black & $1.5 \times 10^{3}$ \\
Koltron G1 [138] & PVDF & Graphene & 2
\end{tabular}

\subsubsection{Conductive materials}

The ability to $3 \mathrm{D}$ print conductors is fundamental to the fabrication of parts with integrated sensors. The conductors might not only be used to fabricate piezoresistive or capacitive sensors but also allow for wiring inside the printed structure to connect the sensors to the read-out electronics.

\section{Filaments}

A common method to directly print with the conductive material in FDM printing is by using a filament made of a conductive polymer composite, e.g. a thermoplastic base material blended with carbon-based materials, such as carbon black, graphene, graphite or carbon-nano-tubes. These materials are low-cost, readily available and chemically stable $[31,124,125,126,127]$. The material is very well suited for the fabrication of truly three-dimensional conductors, where multiple layers of the 3D printed structure are connected. High-filler concentrations will decrease the strength of the materials due to the loss of particle-matrix adhesion [128]. Furthermore, these materials obtain conduction through percolation networks and, therefore, the effect of the filler concentration on the resistivity diminishes with increasing concentration [129, 130, 131]. At last, because of the composite nature of the materials, they often exhibit piezoresistive effects, which is discussed in section 3.3.2. Table 3.1 lists the limited commercially available conductive polymer composite filaments.

\section{Inks}

The inks that can be used for direct ink writing are often specifically developed for a given process, e.g. for ink-jetting or screen printing. Inks for jetting always have a low viscosity and small particle size (1-30 $\mathrm{mPas}$, while screen printing inks generally have a much higher viscosity (1000-10000 mPas) [139]. Since a high solid content increases the viscosity of the ink [140], it can be higher for a screen print ink than for a jetting ink for a given solvent. The viscosity and wetting properties of the used ink determine how it will behave in combination with other materials during printing.

In most common conductive inks silver particles are used. In applications where biocompatibility is a requirement, PEDOT:PSS based inks are often 
used $[141,142]$. The inks come in varying degrees of flexibility, ranging from non-flexible, flexible and even stretchable. Some inks require either a UV or a thermal curing step. For 3D printing applications the stability of the ink is often important, since it is unpractical if ink components separate inside the extruder between prints or even during the print.

\section{Fibers}

Several types of fibers and wires can be used as current carrying traxels for sensing applications. Based on the electrical and mechanical properties, important distinctions can be made. Copper wires can be used for carrying large currents because of the high conductivity of copper, which for wires equals the bulk conductivity [119].

Parts with embedded carbon fiber have already been printed with a resistivity of around $3.7 \times 10^{-4} \Omega \mathrm{m}$ [110]. Carbon fiber can be printed as dry fibers. However, more often, carbon fiber is used in a so-called prepreg, where a bundle of dry fibers is impregnated with a polymer and shaped as a filament beforehand [143]. A prepreg offers advantages for printing since dry carbon fibers are very brittle, difficult to handle and easily introduce void formation and poor adhesion during printing $[108,143]$. On the other hand, dry fibers with co-extrusion give more flexibility for choosing the fiber and matrix combinations. So copper wires can be used in case high currents or low resistance is required, where carbon fiber is mainly used for high yield strength and offers higher resistance.

\section{Liquid metals}

Another class of conductors that can be used to add functionality to 3D printed objects are liquid metals, like gallium indium (eGaIn) and Galistan (eGaInSn). These liquid metals can be added to the printed object using direct ink writing [144, 145] or afterwards by filling [146]. The metals have a low resistivity of $29 \mu \Omega \mathrm{cm}$ in case of Galistan [147], as well as a low viscosity of (2.4 mPa s for Galistan [147]) and because the metal remains liquid it can be stretched, up to $200 \%$ without breaking [144]. It should be noted that liquid metals such as eGaIn corrode other metals and therefore, might cause damage to the printers in which they are used [148].

Which conductive material is most suitable depends on the application, as illustrated in Table 3.2. FDM filaments offer a low-cost solution for the fabrication of actual three dimensional conductors. However, their conductivity is limited. Copper fibers, liquid metals and silver inks allow much higher current densities in stiff structures. Besides their high conductivity, stretchable silver 
Table 3.2: Comparison of extrudable conductors

\begin{tabular}{l|llll} 
& Filaments & Inks & Fibers & $\begin{array}{l}\text { Liquid } \\
\text { metals }\end{array}$ \\
\hline $\begin{array}{l}\text { 3D interconnects } \\
\text { Conductivity }\end{array}$ & Easy & Difficult & Difficult & Moderate \\
Stretchable & Yes & Good & Excellent & Excellent \\
& & Yes & No & Yes
\end{tabular}

inks and liquid metals also can be stretched up to multiple times their length.

\subsubsection{Sensing materials}

Due to their piezoresistive properties many of the aforementioned conductive materials can also be used as sensing material. However, besides piezoresistive materials, piezoelectric and magnetic materials have also been used as a functional sensing material in 3D printed sensors.

\section{Piezoresistive materials}

When a conductive material is strained, the resistance changes due to a change in diameter, length and specific resistivity. However, in most conductive polymer composites, the resistance change is bigger than expected by the geometrical effect due to the piezoresistive effect, resulting in a higher strain sensitivity. Conductive polymer composites show this piezoresistive effect due to the change of conductive paths in the polymer matrix under strain [88]. A major drawback of conductive polymer composites is the nonlinear resistance changes when the material is strained. Usually the resistance first decreases at small strains, due to optimal alignment of the conductive particles in the matrix, and subsequently increases at higher strain [124]. Furthermore, while the material is strained, there is a formation caused by polymer mobility and breakdown of conductive networks. At low strain rates these effects are in balance resulting in minimal resistivity changes, whereas at high strain rates the breakdown effect is more dominant [149, 150]. Furthermore, some used polymers, such as TPU, are viscoelastic themselves [151], which further increases the hysteresis and nonlinearity in the response of the piezoresistors. However, 3D printing offers the benefits of easy customization in size and shape to optimise the gauge factor and strain range of the sensor, besides shape optimisation the filler content could be tuned [124]. Furthermore, it might be possible to model these effects and compensate for them in software, as is discussed further in section 3.6. Besides conductive polymer composites, piezoresistive sensors could also be fabricated using conductive inks [152, 94], liquid metals [153, 154] or conductive fibers $[113,114]$. 


\section{Piezoelectric materials}

A common piezoelectric material used in 3D printing is polyvinylidene fluoride (PVDF). The material can directly be printed [155], and so can PVDF-ceramic composites [156]. The material can also be dissolved and used as ink, with the addition of a co-polymer [157] or ceramic particles [158]. Often the material is polarised after the fabrication process in order to increase the piezoelectric constant [159]. The formation of the piezoelectric $\beta$-crystal structure of PVDF can be enhanced by printing with a high voltage applied between the nozzle and the bed, as shown in $[159,160]$. It should be noted that an effect similar to that of a piezoelectric material can be achieved using an electret, which can be $3 \mathrm{D}$ printed as well [161].

\section{Magnetic materials}

Both soft magnetic thermo-plastics [162] as well as hard magnetic thermoplastics [163] are commercially available. These thermo-plastics can be 3D printed and subsequently poled to produce a material with a remnant magnetisation $[164,165,166]$. Specific magnetic thermoplastic composites can also be fabricated for specific research purposes [167, 168] on magnets and sensors. Another option is to include wires of ferromagnetic materials, such as nickel, for their magnetic properties [115].

Piezoresistive materials offer a simple and low-cost solution for adding pressure sensitivity to 3D printed parts. However, when the 3D printed piezoresistors are made using conductive polymer composites, they often show hysteresis and large nonlinearities. Piezoelectric and hard magnetic materials can be printed. However, for most applications, they need to be poled during or after printing.

\subsection{Bonding technology}

Bonding wires to 3D printed sensors is important because of the significant influence of the connection on the resistance [169]. Despite this importance, it is a largely unexplored field and currently no standard exists. Table 3.3 lists the most important methods used in literature. The 3D printing process specifically enables printing of connector geometries [170] and inserting electrical conductors during manufacturing [169]. The methods have large differences in contact resistance (from $1.6 \Omega$ [110] up to more than $1000 \Omega$ [169]). To bypass the high contact resistance, often four-wire measurements are used [83]. For connectors that are pushed in or onto the sample afterwards, the resistance strongly depends on the applied pressure [169], and therefore the contact 
Table 3.3: Overview of Bonding Technology for 3D printed sensors.

\begin{tabular}{llll}
\hline Bonding Technique & References & Advantage & Disadvantage \\
\hline Silver ink, paste and epoxy & {$[88,110,172,112]$} & Low contact resistance & Can be mechanically weak \\
Embedding wires by melting & {$[89,31,91]$} & Mechanically strong & Risk of deforming sensor \\
Copper tape inserted during printing & {$[169,173]$} & Mechanically strong & High electrical contact resistance \\
Connectors, headers and jumper wires & {$[83,169,170,174]$} & Compatible with standard electronics & Contact resistance pressure dependent \\
3D printed snap-on connector & {$[90,175]$} & Compatible with EMG hardware & Only suited for specific cases \\
Screwed or bolted & {$[176,126,177]$} & Mechanically strong & Bulky \\
Soldering & {$[178]$} & Low contact resistance & Only suited for e.g. electrodeposition
\end{tabular}

resistance influence the measurement. Especially for flexible and stretchable sensors, the connections are still challenging [171]. For connecting to carbon fiber, special steps need to be taken in order to access the fiber and to remove the matrix $[110,114]$.

\subsection{Anisotropic conduction in 3D printed materials}

The line-by-line, layer-per-layer FDM printing process creates anisotropic structures in 3D prints. These structures yield anisotropy in physical properties, which has been studied for e.g. the mechanical [179], thermal [180], magnetic [181] and electrical [182] domains. For the thermal and electrical properties, interfacial or interlayer contact resistance in the build direction in combination with the presence of voids are mentioned as the fundamental reasons for anisotropy $[180,182,176]$. This interlayer contact resistance strongly depends on raster angle, layer height and extrusion temperature [169, 92, 182]. For multiple printed layers, cross-ply infill patterns yield a lower resistivity, possibly because current paths more easily divert around areas of high local resistance [183]. The anisotropic resistivity can also be observed in-plane as a function of the raster angle (infill orientation) [169, 92]. A solution for reducing in-plane anisotropy could be the use of different infill patterns, e.g. by means of a slicer that creates a maze-like (randomized) infill to obtain isotropic macroscopic properties [184]. On the other hand, the anisotropy can be purposely used for improvement of sensors, where inter-layer or inter-traxel resistance aids in sensitivity or directionality [31, 98]. For example, directional anisotropic strain sensors are printed with a meander-like infill, where the sensitivity and anisotropy can be tuned by means of the air gap between traxels, the infill density, and build orientation [98].

Modeling and measurements have been used to gain a better understanding of the anisotropic electrical properties. Hampel et al. [176] presented a model for anisotropic conduction, which assumes a different layer, intra-layer (intertraxel) and inter-layer resistance due to improper fusion in different directions and demonstrated its use with a 1D example. Monte-Carlo simulations have been used to study the effect of local resistance variations in resistor networks 

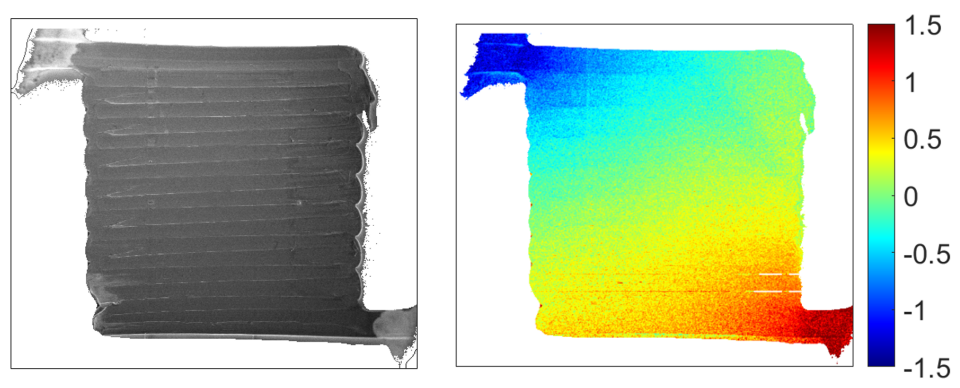

Figure 3.2: VCSEM result (left) and the derived electric potential distribution (right) for a 3D printed sheet with in-plane anisotropic conduction [30] (C)2020 IEEE).

to represent 3D printed conductors [183]. FEM simulations are used to study anisotropy in all directions [182]. A small cell of traxel pieces represents the material with voids and layer bonding. Since voids, raster orientation and layer bonding are important for the anisotropic conductivity [92], these features need to be included in modelling.

Impedance measurements are used for experimental characterization. In this way, inter-traxel and inter-layer resistance can be measured with rectangular samples in-between conductive plates in different orientations [169, 92, 182]. Research indicates that in-situ resistance measurements during printing can also be used for characterization of conductive prints [173]. In-plane anisotropy, however, can also be measured with a voltage contrast method in the scanning electron microscope (SEM) and with infrared (IR) thermography measurements via Joule heating [30]. Voltage contrast SEM (VCSEM) measures potential distributions since a difference in electric potential gives rise to contrast. In Figure 3.2 on the left, the contrast in an SEM image can be seen, whereas, on the right, the derived electric potential distribution is displayed. A small amount of anisotropic conduction is present in this case since the voltage distribution extends more along the traxels than across the traxel's interfaces [30]. During IR thermography measurements, an IR camera measures local temperature increases due to Joule heating, which is linked to the power dissipation. Hence, these two methods can be used to study the anisotropic conduction in sheet-like sensors [30] 


\subsection{Compensation methods}

\subsubsection{Drift compensation}

Drift is the generic name for slowly varying random changes, which can be caused by, e.g., variations in temperature or humidity, unstable power supplies or $1 /$ f noise [185]. In 3D printed sensors, these random errors might be caused by, for example, the positive temperature coefficient of the used piezoresistors [90, $186]$ or the creep of the used polymers $[187,188]$. In order to compensate for this drift, a differential measurement might be used [28], or a separate temperature measurement can be used to compensate for this effect [189].

\subsubsection{Nonlinearity compensation}

Soft polymers have been shown to exhibit strong hysteresis [190], time dependence, as well as cyclic softening [151]. It is shown that the effects of these nonlinearities are even more emphasized towards resistive sensors [191]. Compensation methods are then required before the application range of such sensors can be extended. Focusing on FDM manufactured sensors, it is shown that a differential measurement significantly decreases the nonlinear response of 3D printed strain sensors [28]. Additionally, Hysteresis can be simulated by a mathematical model, those models use a similar working principle: a set of experimental measurements is obtained on which the model parameters are fitted in an identification procedure [192]. Most often, the models are designed such that they capture the behavior of a given system, the nonlinear response can then be compensated with the use of the inverse of the model [193, 194, 195]. Alternatively, the model can also directly be fitted to the inverted data, as was shown by Schouten et al. [196].

\subsection{Examples of 3D printed sensors}

Many of the conductors used in traxel based 3D printed sensors have a large piezoresistive constant and are therefore ideal for use in force and tactile sensing applications [81]. These sensors have been fabricated using co-printing with conductive pastes such as the bending sensor demonstrated by Nassar et al. [152] that uses silver palladium paste as the functional material. Gue et al. [94] demonstrated a small tactile sensor that is capable of sensing a person's pulse using a custom-made ink by using a mixture of silver particles and silicone elastomer. More practical applications of strain sensors can be found on sensing 
structures that use conductive polymer composites as functional material, such as tactile and touch sensors printed using soft conductive and nonconductive thermoplastic polyurethane (TPU) materials [89, 90]. These TPU materials allow for high flexibility, as shown in a proof of concept glove for measuring finger flexion by Christ et al. [87]. Kim et al. [88] demonstrated a multi-axial force sensor consisting of a $3 \mathrm{D}$ cross that allows for sensing forces in $x, y$ and $z$ direction using the same type of materials.

In comparison to piezoresistive sensing methods, capacitive sensing methods have the advantage that the drift, nonlinearity and hysteresis in the conductive elements do not influence the measurement. The ability to $3 \mathrm{D}$ print very soft materials allows the fabrication of force sensors that consist of a parallel plate capacitor with a dielectric that is directly compressed $[116,34,186,197]$. It should be noted that capacitive sensors often need shielding or guarding in order to reduce the influence of parasitic capacitances. Another commonly used technique is to print a conductive structure that forms a capacitive structure, which can detect changes in the dielectric constant of the materials in its environment. E.g., the capacitance will be changed by the presence of a finger, wood, glass or steel [198, 186].

When printing technologies support the fabrication of conductors with a high conductivity, inductive sensing can become another measurement principle on which 3D printed sensors can be based on. At the moment the limited conductivity of carbon-based filaments is generally not sufficient for serving as inductor $[126,199]$. 3D printed inductors can e.g. be fabricated by means of directly printing with copper infused Electrifi filament (see Table 3.1 ) [126] or by electroplating onto Electrifi material [178]. Other inductive coils and sensors are printed by means of embedding copper and nickel wires [115], by filling a 3D print with silver paste [200] or liquid metal [201] or by spraying a graphenefilament coil with silver nano ink [199].

Another approach is by making use of the anisotropy in the structures fabricated using FDM 3D printing. Such an example (Figure 3.3) is presented in [31] and makes use of the high inter-layer contact resistance. Due to this poor resistive coupling capacitive effects between layers become prominent, allowing for the fabrication of capacitive force sensors by depositing only two thin layers of material.

Figure 3.4 show a systematic analysis of this sensor using a linear actuator to apply a cyclic load between $0 \mathrm{~N}$ to $10 \mathrm{~N}$. The force shows a clear square wave pattern, as applied by the actuator. The displacement and capacitance, however, show a damped behaviour, especially after unloading caused by the viscoelastic properties of the used TPU material. Furthermore, these material 


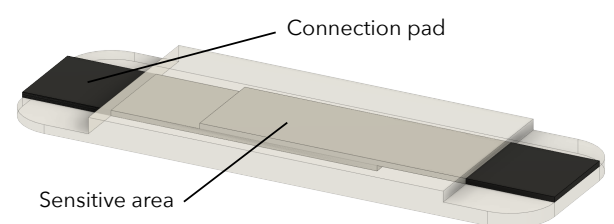

Figure 3.3: CAD drawing of the concept sensor, black parts are conductive parts, gray parts are dielectric [31].

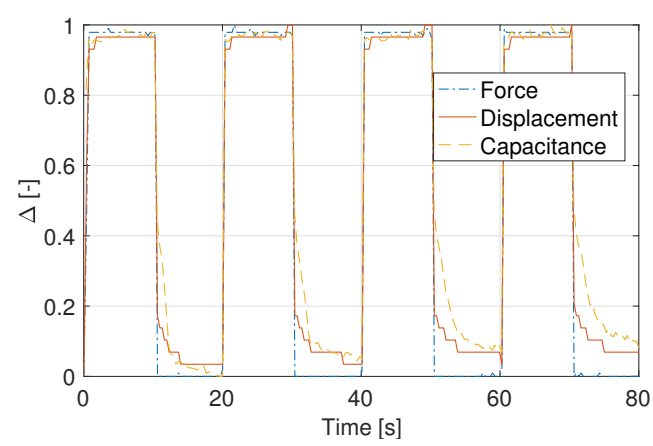

Figure 3.4: Relative change of the applied force and measured displacement and capacitance. The applied force was a square wave ranging from $0 \mathrm{~N}$ to $10 \mathrm{~N}$.

properties are also reflected in the nonlinear capacitance to force behaviour shown in Figure 3.5 and 3.6, where the sensor shows a bigger capacitance change for lower forces than for larger forces.

\subsection{Conclusion}

Several traxel based 3D printing technologies exist to extrude filaments, inks and fibers. However, there are still challenges in the switching between materials to limit contamination and misalignment between materials. The extrusion of flexible filaments is ideally done using a direct-drive extruder. Fiber placement and co-extrusion both appear to be well suited for the fabrication of $3 \mathrm{D}$ printed sensors, however, printing fibers outside of the $x-y$ plane is still challenging.

$3 \mathrm{D}$ printing is already being used to print a wide variety of electro- and biomechanical sensors where piezoresistive sensors offer a simple and cheap way to integrate sensing. Capacitive sensors on the other hand are able to provide more linear sensors in exchange for added complexity. Additionally, sensors can be fabricated by making use of the anisotropy of the 3D printed structures. 


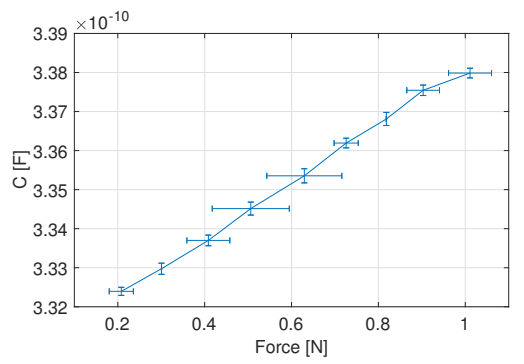

Figure 3.5: Mean capacitance and mean force including standard deviations for low forces.

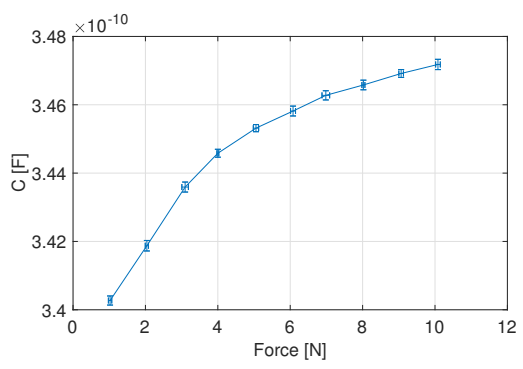

Figure 3.6: Mean capacitance and mean force including standard deviations for high forces.

The design freedom offered by 3D printed sensors provides new opportunities for development of integrated sensing structures. However, due to the use of soft polymers, 3D printed sensors show many nonlinearities. Understanding these limitations and the use of compensation algorithms is important to enable the fabrication of improved sensors that can be used in biomedical, soft robotics and many more applications. 


\title{
Chapter 4
}

\section{D printed soft sEMG sensing structures}

\begin{abstract}
D printing of soft surface electromyography (sEMG) sensing structures enables the creation of personalized sensing structures that can be potentially integrated in prosthetic, assistive and other devices. The chapter covers the development and characterization of flexible carbon-black doped TPU-based sEMG sensing structures. The structures are directly 3D printed without the need for an additional post-processing step using a low-cost, consumer grade multi-material FDM printer. A comparison between the gold standard $\mathrm{Ag} / \mathrm{AgCl}$ gel electrodes and the 3D printed EMG electrodes with a comparable contact area shows that there is no significant difference in the EMG signals' amplitude. The sensors are capable of distinguishing a variable level of muscle activity of the biceps brachii. Furthermore, as a proof of principle, sEMG data of a 3D printed 8-electrode band are analyzed using a patten recognition algorithm to recognize hand gestures. This work shows that 3D printed sEMG electrodes have great potential in practical applications.
\end{abstract}

This chapter is published as: G. Wolterink, P. Dias, R. Sanders, F. Muijzer, B.J. van Beijnum, P Veltink, G. Krijnen, Development of soft sEMG sensing structures using 3D printing technologies, Sensors 20 (15), 4292, 2020 


\subsection{Introduction}

Electromyography (EMG) is a technique for measuring the electrical activity produced during muscle contraction. EMG provides useful information about the activity of individual muscles and is therefore widely used in the control of hand and arm assistive devices and prosthesis [202, 20, 21, 22, 23, 203, 204].

Surface electromyography (sEMG) electrodes are currently only available in fixed shapes. Some flexible structures are available, such as the TMSi High Density EMG grids [32]; however, these structures cannot be stretched to adapt to complex anatomy. Therefore, it is often still necessary to place electrodes individually. For everyday patient use of EMG assistive technologies, personalized sensing structures are desirable. Additive manufacturing, best known from 3D printing, enables the creation of personalized low-cost structures, making for a useful tool for prosthetic assistive devices [86]. With the advent of multi-material printers, materials varying in conductivity and mechanical properties can be co-printed, enabling the creation of personalized sensing structures [91, 83]. Therefore, 3D printed sEMG sensing structures will require minimal assembly and are easily scalable. Such structures can be soft and follow body contours, thus not influencing musculoskeletal deformations and greatly improving the comfort of the user and aesthetics of the device.

Although other methods, such as conductive fabrics [205] or screen printing [206], allow for the fabrication of flexible customized sEMG sensors, 3D printing has the potential to directly print assistive devices and prostheses that incorporate embedded and customized sEMG electrodes that are directly 3D printed without the need for additional post-processing steps. Furthermore, these structures could also incorporate other types of 3D printed sensors, such as force or tactile sensors [81, 31, 91], and include 3D wire routing and shielding.

The goal of this study is to explore the potential use of additive manufactured EMG electrodes made of flexible carbon doped thermoplastic polyurethane by comparing their performance to conventional electrodes. Furthermore, a multielectrode setup for use in real-time gesture detection is shown as a proof of principle application for 3D printed electrodes.

\subsubsection{EMG}

EMG can be recorded either invasively or non-invasively; sEMG gives information about the global muscle activity and is preferable to intramuscular EMG (iEMG) because of its non-invasive nature. The bandwidth of an sEMG signal lies between 0 and about $500 \mathrm{~Hz}[207,20,208]$. The signal values are in the 
range between a few microvolts to a millivolt depending on the location and the electrodes [21, 20]. Peak values lie in the range between 0.1 and $1 \mathrm{mV}$ [207].

In the body, current is facilitated by ion transport. sEMG electrodes convert the ionic current within the tissue into an electronic current. The electrodes can be classified into two main types: dry and wet electrodes [20]. Both wet and dry electrodes have their particular advantages and disadvantages.

The most commonly used electrodes are silver/silver chlorides gel-electrodes (AgCl electrodes). These wet electrodes approximate a perfectly non-polarizable electrode, which is characterized by the free flow of charge between the electrode and the body [20]. AgCl electrodes are widely available and inexpensive $[209,210]$. Such electrodes are relatively stable when used in biopotential measurements due to the chemical equilibrium reaction between the silver $(\mathrm{Ag})$ electrode and the silverchloride $(\mathrm{AgCl})$ coating, which provides a stable electrochemical interface between ionic medium and electrode [207]. However, $\mathrm{AgCl}$ electrodes are less suited for long-term use, since the electrolytic gel will dehydrate over time and this will influence the signal quality [20, 210, 211]. Another drawback is that some people are sensitive to the electrolyte gel, causing skin irritation and other allergic reactions [211].

Dry electrodes usually have a higher electrode-skin impedance compared to wet electrodes and are more sensitive to movement artifacts [211]. Dry electrodes have the advantage of being reusable and are more suited for long-term measurements [210]. In electrode arrays, dry electrodes are preferred since electrodes are placed close to each other and conductive gel could create conductive pathways, short-circuiting the electrodes [212]. Dry electrodes can be fabricated using various methods and materials. This makes them useful for integrating into prostheses and other devices. The literature shows electrodes made from semiconductor materials, gold-coated 3D prints [211], metal or metal filled polymers [213], conductive fabrics [214], printed circuit boards (PCB) [210], silver inks printed on flexible polyamide films [23] and carbon-filled rubbers [213, 215].

Several studies have shown that dry electrodes can give results comparable to wet electrodes after only a few minutes [216, 217]. Pylatiuk et al. [216] performed impedance measurements over $3 \mathrm{~h}$ on dry and wet electrode. The measurement showed an increase in skin-electrode impedance for the wet electrodes after 30 min, due to evaporation of the electrolyte. After $150 \mathrm{~min}$, the impedance of these electrodes was higher than that of the dry electrodes, resulting in poorer signal qualities. Dry electrodes showed a high skin-electrode impedance directly after application $(100 \mathrm{k} \Omega$ to $400 \mathrm{k} \Omega$ ). Due to sweat and moisture build-up, the impedance decreased to values ranging from $40 \mathrm{k} \Omega$ to $80 \mathrm{k} \Omega$ after $20 \mathrm{~min}$. Over the long term, dry electrodes even have a stabler impedance, since wet electrodes 
dry out over time $[216,218,214]$.

Due to the relatively high electrode-skin contact impedance, biopotential amplifiers need to have a high input impedance. Webster et al. stated an average contact impedance of $1 \mathrm{~cm}^{2}$ skin of around $200 \mathrm{k} \Omega$ at $1 \mathrm{~Hz}$ to $200 \Omega$ at $1 \mathrm{MHz}$ [207]. Biopotential amplifiers nowadays have input impedances ranging over $100 \mathrm{M} \Omega$ [219]. An input impedance that is as high as possible is preferred since this also reduces noise caused by impedance mismatch, which generates a differential signal of the common mode voltage. Other noise prevention methods include (active) shielding of the wires between the electrode and the amplifier.

When 3D printing electrodes, it is preferable to make dry electrodes, since dry electrodes are more suited for long-term measurements and because of hygienic reasons. Since biopotential amplifiers nowadays have large input impedances, 3D printed electrodes do not need to be made from highly conductive materials.

\subsubsection{Conductive 3D Printing}

Both 3D printing technologies and the number of available materials have grown significantly in recent years, while the cost of both continues to decrease. To 3D print sensing structures, dielectric and conductive materials need to be combined into single structures. Two main methods to embed a conductive material into a 3D printed structure are filling and direct printing.

The first method adds conductive properties to the material by filling channels or cavities in a printed object with a conductive filler. Filling is usually an extra processing step that takes place after the printing process [220, 221, 222]. The advantages of this method are the wide variety of conductive fillers and printing techniques that are available. However, most conductive fillers have a high viscosity, resulting in large pressure drops and long filling times, limiting the channel maximum length, minimal cross-section and complexity. Additionally premature solidification of the filler can strongly hamper the filling process. The creation of extensive conductive networks is furthermore limited by the difficulties involved in the removal of the channel support material and complications such as the formation of bubbles [223].

Direct conductive 3D printing has been demonstrated with the use directink writing using silver particle infused inks or elastomers [131, 152, 94, 224]. Fused deposition modeling (FDM) and stereo lithography (SLA) are commercially available printing technologies that allow direct conductive 3D printing. SLA is a photo-curing printing method where a liquid resin is solidified layer by layer. Although flexible conductive SLA-printed structures, based on ionic com- 
posite hydrogels, have been demonstrated [225], this technology is currently not suitable for the development of multi-electrode structures, since SLA is limited to a single material [81]. FDM is an extrusion-based 3D printing technology in which a thermoplastic material in the form of a filament is pushed through a heated nozzle in which the material is melted. The nozzle deposits a thin layer of molten material on a predefined path in the $x y$-plane. To create structures extending in the third dimension ( $z$-direction) as well, the process is repeated, building up the model layer by layer. To enable the deposition of multiple materials, the printer is fitted with multiple extruders and nozzles.

Models made using FDM printing are usually made from a rigid thermoplastic, such as polylactic acid (PLA), acrylonitrile butadiene styrene (ABS), glycol-modified PET (PETG), polyvinyl alcohol (PVA) or nylon. However, there is a growing variety of flexible materials available. Most of these materials are thermoplastic polyurethanes (TPU); commonly know materials are NinjaFlex and PolyFlex [137, 226]. Softer flexible materials are X60 [227] and the Lay-Fomm or Lay-Gell filaments, which become flexible after dissolving the PVA compound, leaving a porous soft structure [228].

To print conductive structures using FDM printing technologies, the raw thermoplastic material is blended with conductive particles such as carbon black. Currently only a few conductive materials are available; see Table 3.1. PIETPU is currently one of two commercially available filaments that have flexible properties (Young's modulus of $12 \mathrm{MPa}$ ) and a low shore hardness of $95 \mathrm{~A}$ [229].

An increase of the volume fraction of the conductive particles will reduce the resistivity of the material as modeled by percolation theory. The model predicts that the resistivity of the material $(\rho)$ decreases in response to an increasing filler concentration $(m)[129,230,131]$ :

$$
\rho=\rho_{0}\left(m-m_{\mathrm{c}}\right)^{-\beta} \quad \text { for } m>m_{\mathrm{c}}
$$

where $m_{\mathrm{c}}$ is the critical concentration of the conductive filler. The value of the critical exponent $(\beta)$ depends on the type of particles. Since the decrease of the resistivity is exponential, the effect of the conductive filler concentration will become substantially less as the concentration increases. Due to the decrease in elastic properties in response to increasing filler content [128], a compromise between the resistivity reduction and flexibility needs to be made. 

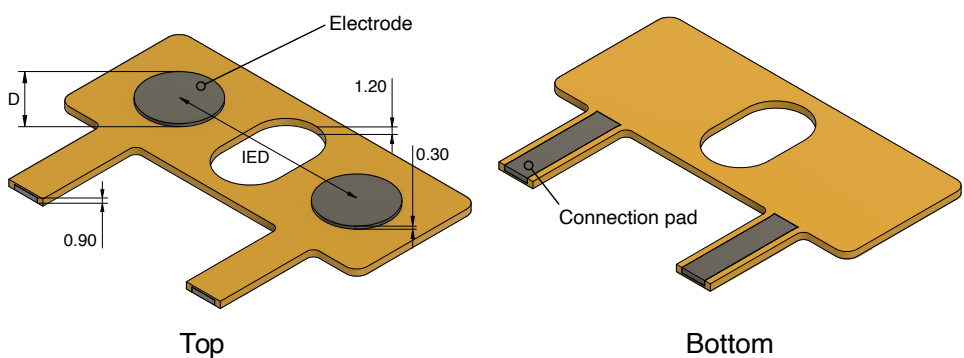

Figure 4.1: CAD drawing of the electrodes; grey parts are conductive, orange part are dielectric. The dimension are indicated in $\mathrm{mm}$. The inter-electrode distance (IED) and the diameter (D) are variable and listed in Table 4.1.

\subsection{Methods}

\subsubsection{Electrode Impedance}

To gain insight into the impedance of the EMG electrodes, a pair of 3D printed electrodes (D10_IED20) and $\mathrm{AgCl}$ electrodes were placed on a cloth rinsed in a $0.9 \% \mathrm{NaCl}$ solution. The measurement was performed in three- and four-point configurations using an LCR meter (HP4284A). The measurement frequency ranged from $20 \mathrm{~Hz}$ to $2.5 \mathrm{kHz}$.

\subsubsection{Side-by-Side sEMG Electrode Characterization}

To characterize the performance of 3D printed electrodes compared to commercial electrodes, and to gain insight into the influence of electrode size on the EMG signal, sensing structures with varying geometry were 3D printed. These sensing structures were designed using Fusion 360 (Autodesk, San Rafael, USA). Figure 4.1 shows a CAD drawing of the sensing structures. Parts shown in dark gray are made from conductive PI-ETPU, whereas the orange parts are made from non-conductive Ninjaflex and are used as insulation and to keep the electrodes at a fixed distance. Both PI-ETPU and NinjaFlex are flexible materials with a Young's modulus of $12 \mathrm{MPa}$ and a Shore hardness of 95A for PI-ETPU and $85 \mathrm{~A}$ for NinjaFlex. The sensing structures of various electrode diameters and inter-electrode distances (IED) are listed in Table 4.1. The thicknesses ( $z$ direction) are equal for all sensing structures and are indicated in Figure 4.1. The material cost of the sensing structures is estimated to be between $€ 0.10$ for the smallest and $€ 0.20$ for the largest structure.

The electrode structures were printed using a modified FlashForge Creator 
Table 4.1: Sensing structures and corresponding dimensions. The electrodes' names of the bi-polar 3D printed electrode represent their size, i.e., D16_IED20 has a diameter (D) of $16 \mathrm{~mm}$ and inter-electrode distance (IED) of $20 \mathrm{~mm}$.

\begin{tabular}{lcc}
\hline Name & Diameter $(\mathbf{D})[\mathbf{m m}]$ & Inter-Electrode \\
\hline AgCl & 16 & 20 \\
D16_IED20 & 16 & 20 \\
D16_IED30 & 16 & 30 \\
D10_IED20 & 10 & 20 \\
D10_IED30 & 10 & 30 \\
D05_IED20 & 5 & 20 \\
D05_IED10 & 5 & 10 \\
Snap & 25 & - \\
\hline
\end{tabular}

Pro FDM printer (FlashForge Corporation, Zhejiang, China), fitted with two direct drive extruders suited for printing flexible materials (Flexion Extruder, Diabase Engineering, Longmont, USA). A personal computer running slicer software (Simplify3D, Inc., Cincinnati, USA) sliced the CAD model and managed the control of the printer. The structures were printed with a layer height of $150 \mu \mathrm{m}$ To compensate for possible errors in the bed leveling, the first layer was printed at a layer height of $300 \mu \mathrm{m}$. The nozzle diameter used to extrude NinjaFlex was $600 \mu \mathrm{m}$; PI-ETPU was printed using a wider nozzle of $800 \mu \mathrm{m}$ to prevent blockage. Connections to the sensing structures were made by melting a stranded copper wire onto the connection pads. Shrink tubing was used for insulation and strain relief. The other side of the $20 \mathrm{~mm}$-long unshielded wire interfaces with the shielded wires of the biopotential amplifier. Figure 4.2 (left) shows an assembled D10_IED20 electrode

For single electrode applications, a snap button electrode was designed; see Figure 4.2 (right) and Figure 4.3. This type of connector allows for easy connection since the snap button is a standardized connection used in many commercial EMG systems.

\section{Study Design}

Six healthy adult $(18+)$ volunteers participated in the measurements, which were approved by the faculty ethics committee. Before application of the EMG sensing structures, the skin around the biceps brachii is prepared according to SENIAM recommendations $[209,231]$. If hair is present, the skin is shaved. Next, both the skin and the printed electrodes are cleaned using alcohol. After the alcohol has evaporated, the sensing structures are placed above the biceps brachii according to SENIAM recommendations. The printed snap electrodes 

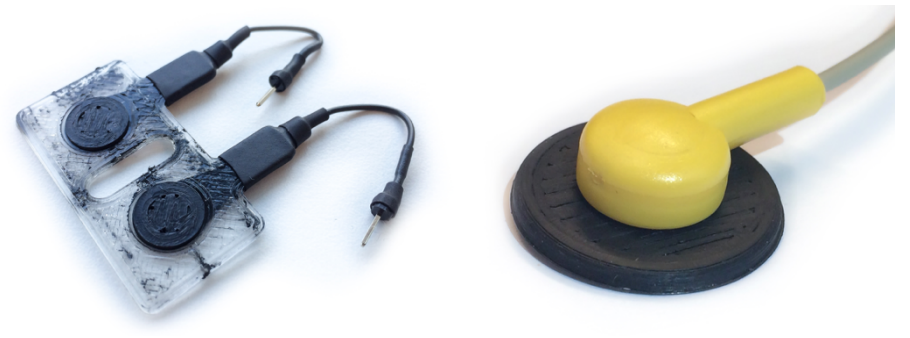

Figure 4.2: Left: 3D printed and assembled sensing structure (D10_IED20). Right: Connected 3D printed snap electrode.
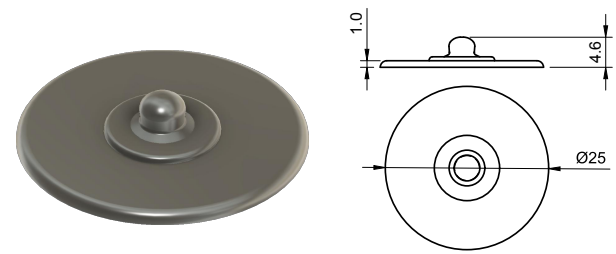

Figure 4.3: CAD drawing and dimensions of the snap electrode.

function as patient ground and are placed on the wrist of the opposite arm. To provide better electrode-to-skin contact, the electrode is moistened using a cotton swab dipped in tap water. The electrodes are connected to a TMSi Refa (Twente Medical Systems International B.V., The Netherlands) biopotential amplifier. This amplifier is connected to a computer via a bidirectional optical fiber. The EMG data are sampled at $1250 \mathrm{~Hz}$, and are visualized and stored using the TMSi Interface for Matlab.

For the first measurement, the subjects were asked to perform three short isometric contractions of $1 \mathrm{~s}$ per contraction. To help with the isometric contraction, the subjects were asked to try and lift a table fixed to the floor. The voluntary contraction was measured by asking the subjects to perform elbow flexion followed by extension without any load (concentric contraction); this movement was repeated 3 times. The data obtained from this recording were shown to the researcher in real-time and used to validate the setup and electrode placement.

The second measurement was the load test. The subjects were asked to place their elbow on an armrest and to hold a handle that was attached to a load; see Figure 4.4. At the start of the measurement, the subjects had to keep this position with no load on the handle. After $10 \mathrm{~s}$ the load was increased by $1 \mathrm{~kg}$ and the subjects had to keep their arm in position, causing 


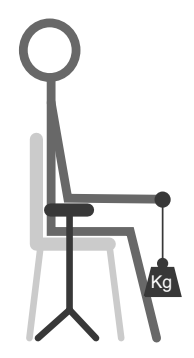

Figure 4.4: Position of the subject performing the load experiment. The elbow is placed on an armrest, and the load was increased from $0 \mathrm{~kg}$ to $5 \mathrm{~kg}$ every $10 \mathrm{~s}$.

the biceps to contract. Every $10 \mathrm{~s}$, the load was increased by $1 \mathrm{~kg}$ until the total load reached $5 \mathrm{~kg}$. Recordings from these tests give information about the signal properties at various loads and help to determine if the printed sensing structures are capable of detecting several levels of muscle activity.

During both measurements, the subjects remained seated. Both measurements were repeated for different electrode setups (though in all setups the snap electrode was used as the patient ground). The first set of measurements compared the gold standard $\mathrm{AgCl}$ electrodes (Covidien, Ireland) and the 3D printed electrodes. Therefore, the $\mathrm{AgCl}$ and printed sensing structures were placed side-by-side on the biceps (Figure 4.5). The $\mathrm{AgCl}$ electrodes were placed next to each other, resulting in an IED of $20 \mathrm{~mm}$. The cross-section of the electrolyte is $16 \mathrm{~mm}$, and therefore the comparison was made using the D16_IED20 printed electrode. The measurements were repeated one by one with various sets of sensing structures as listed in Table 4.1. The structures were tested in both dry and in wet conditions; for the wet condition the electrode was moisturized using a cotton swab dipped in tap water.

\section{Data Processing}

The data were loaded into Matlab and further processed. First, the drift and movement artifacts were removed from the signal by a $20 \mathrm{~Hz}$ corner frequency high-pass filter (3rd-order Butterworth). Next, a $50 \mathrm{~Hz}$ notch filter was applied to the signal. The higher frequencies were filtered out using a $250 \mathrm{~Hz}$ corner frequency low pass-filter (3rd-order Butterworth). Finally, the envelope of the signal was taken using a moving RMS window of 25 samples. The data from the load test were cut into windows of $5 \mathrm{~s}$, each representing a load ranging from $0 \mathrm{~kg}$ to $5 \mathrm{~kg}$. The first window starts at $2.5 \mathrm{~s}$, the second at 12.5 , and so on. To compare the signals, the mean and the standard error of the RMS values were 


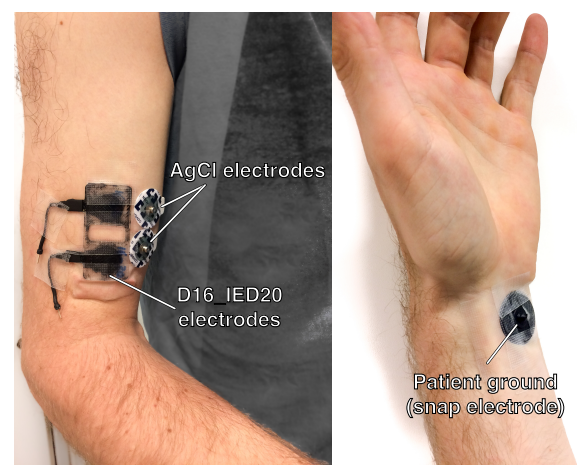

Figure 4.5: Placement of the electrodes on the subject biceps and wrist.

calculated for each load. To compare between subjects the mean of each load sample was normalized using the mean of the $5 \mathrm{~kg}$ load sample of each subject.

\section{Statistical Analysis}

From the time domain data, the mean $(\mu)$ is given by the sum of all elements $\left(x_{i}\right)$ of the envelope divided by the number of elements $(n)$ :

$$
\mu=\frac{1}{n} \sum_{i=1}^{n} x_{i}
$$

To prove, or reject, the difference between $\mathrm{AgCl}$ and $\mathrm{TPU}$ electrodes, the mean envelope per electrode was taken per subject of the $5 \mathrm{~kg}$ sample. Next, the values of the $\mathrm{AgCl}$ and the 3D printed electrode were tested using a paired $t$-test. The increase in muscle activity was proven by taking the mean envelope per subject, per load, of one electrode and testing this against the values of the next load using a paired $t$-test. The same statistical analysis procedure was performed between the various printed electrodes.

\subsubsection{Functional Analysis: Classifier Approach}

To demonstrate a real life application, the $3 \mathrm{D}$ printed electrodes, in eightelectrode-structure, were used in a real-time gesture detection test. The electrodes were placed on the lower arm to detect six classes representing six hand positions, as shown in Figure 4.6: rest, fist, hand extension, wrist flexion, wrist extension and pinch grasp. Due to size limitations of the 3D printer, the 8-electrode armband was made by combining two 4-electrode structures, as illustrated in Figure 4.7. Each of these structures' dimensions are $135 \mathrm{~mm}$ by 


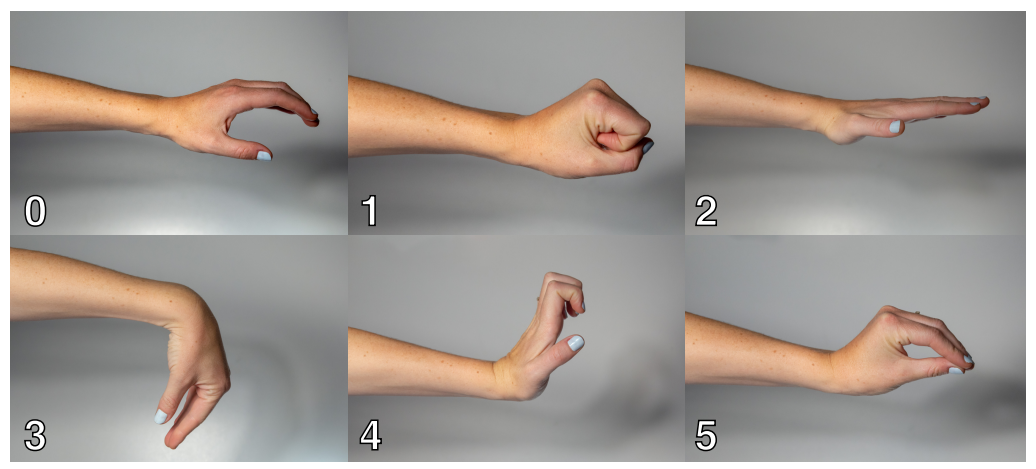

Figure 4.6: The six classes (0-5): rest, fist, hand extension, wrist flexion, wrist extension and pinch grasp.

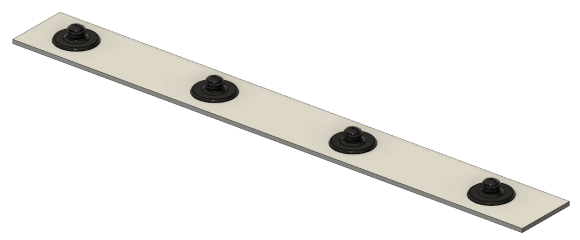

Figure 4.7: Armband design: the 8-electrode armband consists of two of the illustrated structures.

$15 \mathrm{~mm}$ by $1 \mathrm{~mm}$ in which the electrodes, with a diameter of $10 \mathrm{~mm}$, are equally spaced.

Figure 4.8 presents the pipeline followed from the recording of EMG signals to one of the six classes as shown in Figure 4.6. The signals are filtered using a discrete-time, infinite impulse response (IIR) fourth-order $5 \mathrm{~Hz}$ to $450 \mathrm{~Hz}$ bandpass Butterworth filter of direct-form II [232]. Next, the signal is segmented, using $50 \%$ overlapping windows of $200 \mathrm{~ms}$. In each window, data segmentation is performed through the computation of the mean absolute value of each of the sensors.

Classification of movements was performed by using linear discriminant analysis (LDA) and support vector machine (SVM) algorithms. The two algorithms were chosen since LDA is fast and has low computational effort, and SVM is more powerful and might allow for better recognition between gestures.

The functional evaluation of the $3 \mathrm{D}$ printed electrode structure is based on the classification accuracy of the 3D printed eight-electrode structure in comparison to eight $\mathrm{AgCl}$ electrodes. Three healthy adult (18+) volunteers participated in the measurement, which was approved by the faculty's ethics 


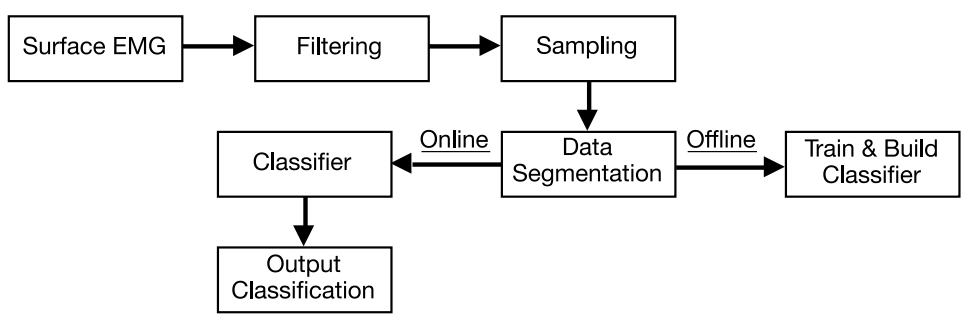

Figure 4.8: Pipeline from signal acquisition to classification.

committee. The subjects were placed in a comfortable position, either sitting or standing, but with the notion that all the movement training should be performed with the arm in the same position. The skin was prepared according to SENIAM recommendations [231] and the electrode bands were placed on the right arm below the elbow. Each electrode was connected to the EMG amplifier. The patient ground of the device, consisting of a damp cloth wristband with silver wires, was placed on the left arm. Next, the following protocol is executed:

- Inactivity: for $9 \mathrm{~s}$, the subject stands still to obtain the EMG in the neutral position.

- The following process is repeated for each class:

- Determining maximum voluntary contraction (MVC): 9 s recording with $3 \mathrm{~s}$ of inactivity and $6 \mathrm{~s}$ of full contraction.

- $3 \mathrm{~s}$ of inactivity followed by $6 \mathrm{~s}$ of contraction at $30 \%, 50 \%$ and $70 \%$ of the previously determined MVC. The subject receives real-time feedback about the target EMG and MVC.

- Offline generation of the classifier: building the LDA and SVM classifiers. Performing a 10-fold cross validation using the training data to obtain the confusion matrix.

- Online testing of the classifier: real-time EMG data are analyzed by the classifier to get real-time feedback of the user's gesture.

This procedure was repeated twice, once for the printed electrodes and once for the $\mathrm{AgCl}$ electrodes. 


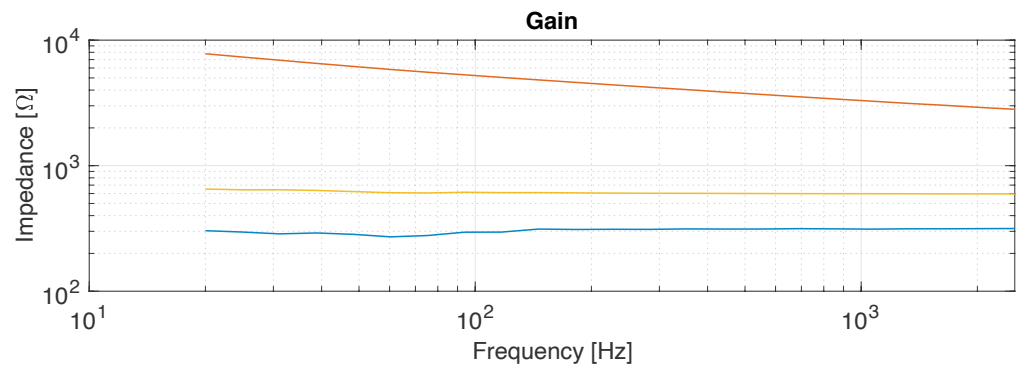

Phase

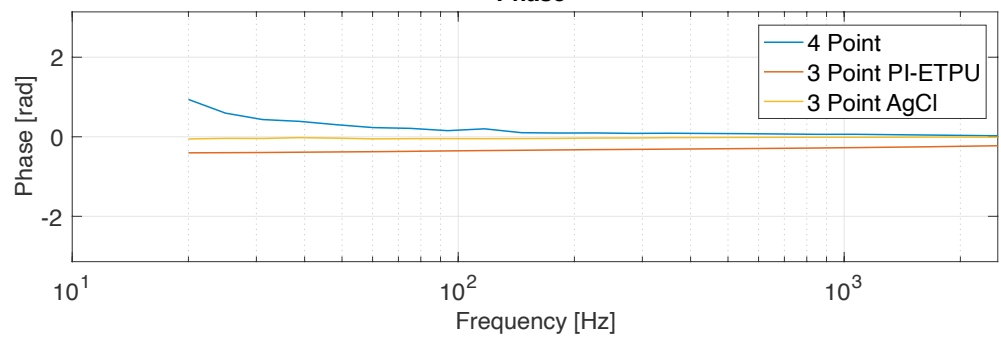

Figure 4.9: Impedance of the ETPU and $\mathrm{AgCl}$ electrodes in contact with $0.9 \% \mathrm{NaCl}$ solution (3-point) and $0.9 \% \mathrm{NaCl}$ solution (4-point).

\subsection{Results}

\subsubsection{Impedance Measurements}

Figure 4.9 shows the result of the impedance measurements in the three- and four-point configurations. Clearly, the 3-point measurements indicate a higher impedance compared to 4-point measurements, which only showed the impedance of the $0.9 \% \mathrm{NaCl}$ solution, pointing to high contact impedance.

\subsubsection{Side-by-Side sEMG Electrode Characterization}

\section{Printed vs. Conventional AgCl Electrodes}

The time-domain data captured using the D16_IED20 and the AgCl electrodes for a given subject, performing three isometric contractions followed by three concentric contractions, are shown in Figure 4.10. The figures suggest that the signals were highly correlated. The noise in the envelope plot was around $15 \mu \mathrm{V}$ and the isometric contractions peaked at $250 \mu \mathrm{V}$. The concentric contractions peaked at just over $250 \mu \mathrm{V}, 370 \mu \mathrm{V}$ and $440 \mu \mathrm{V}$. 

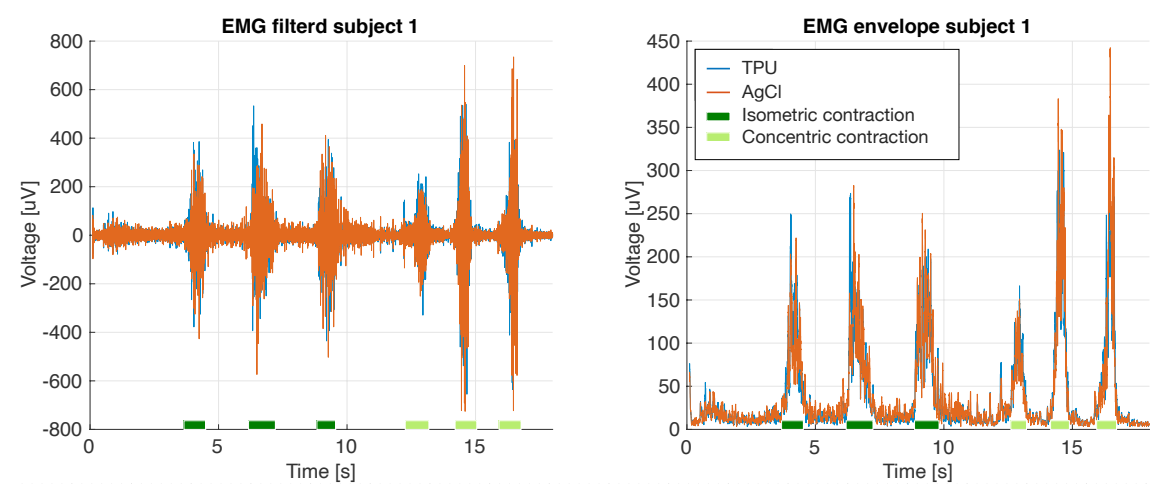

Figure 4.10: EMG measured by the printed D16_IED20 and AgCl electrodes for a given subject performing three isometric contractions followed by three concentric contractions.

Figure 4.11 shows the filtered signal and the corresponding envelope of one subject performing the load test, measured using the printed electrode. The mean envelope and the corresponding standard deviation per subject per electrode are shown in Figure 4.12. For all subjects, except subject 3, the mean envelope of the $\mathrm{AgCl}$ was significantly $(p<0.01)$ higher; for subject 3 the printed electrodes mean envelope was significantly higher $(p<0.01)$. Figure 4.13 shows the mean EMG normalized using the $5 \mathrm{~kg}$ load sample. The normalized mean envelope value increased significantly between each load sample for both the TPU $(p<0.02)$ and $\mathrm{AgCl}(p<0.05)$ electrodes.

\section{Electrode Size}

Tests using the smallest electrode structures with an inter-electrode distance of $10 \mathrm{~mm}$ (D05_IED10) in both wet and dry conditions only captured EMG from one subject; therefore these data are not shown in the results. A comparison between electrodes per subject lifting a $5 \mathrm{~kg}$ load is shown in Figure 4.14. For subject 2 the EMG signals could not be measured with the smallest electrode structure (D05_IED20) under both dry and wet conditions. Overall the figure shows no clear trend in performance differences between electrode sizes, IED and between dry and wet conditions.

Figure 4.15 shows the mean envelope, normalized using the $5 \mathrm{~kg}$ load sample, over all subjects, per weight. Figure 4.16 shows the difference per electrode between weights and corresponding $p$-value. These results show that most measurements using the largest electrodes with a diameter of $16 \mathrm{~mm}$ (D16_IEDXX_XX) were capable of detecting a significant increase in muscle 


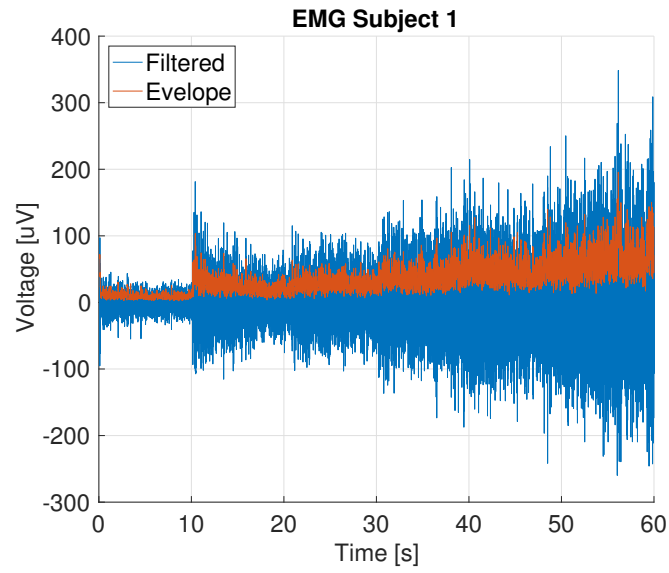

Figure 4.11: EMG recording and its envelope from the load test of subject 1 (D16_IED20 electrode); the load was increased from $0 \mathrm{~kg}$ to $5 \mathrm{~kg}$ in ten second intervals.

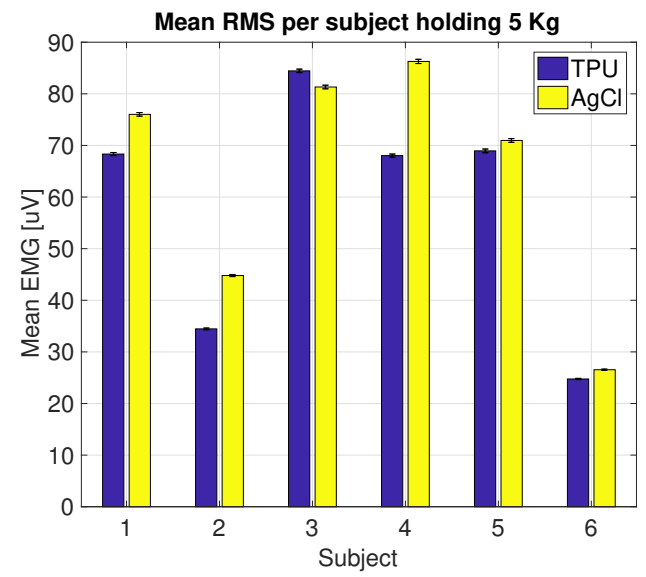

Figure 4.12: Normalized mean envelope value and standard deviation of all subjects performing the $5 \mathrm{~kg}$ load test. 


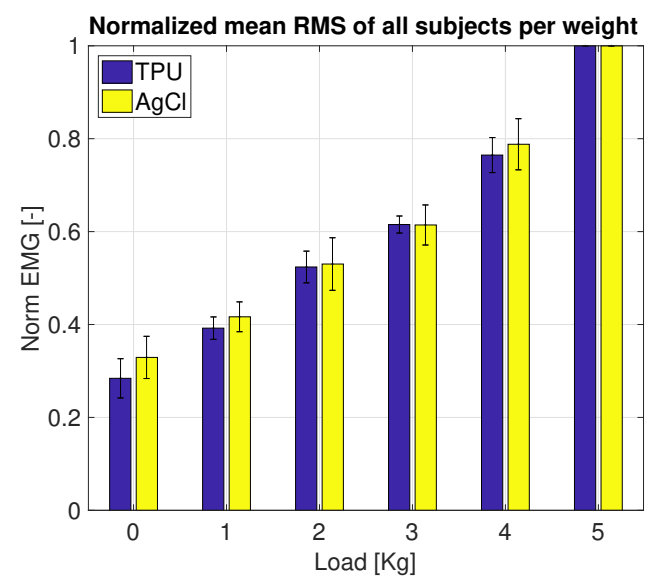

Figure 4.13: Mean envelope and standard deviation of all subject per load.

activity when the load was raised by $1 \mathrm{~kg}$. The medium-size electrodes with a diameter of $10 \mathrm{~mm}$ (D10_IEDXX_XX) had less observed significant differences. For the smallest size (D05_IED20_XX), increases and decreases in the mean envelope were most probably coincidental. The difference in mean envelope between $0 \mathrm{~kg}$ to $5 \mathrm{~kg}$ load data for electrodes of diameters $16 \mathrm{~mm}$ and $10 \mathrm{~mm}$ was significant. However, for electrodes of a $5 \mathrm{~mm}$ diameter, these differences were less significant; see Figure 4.16 for $p$-values.

\subsubsection{Functional Analysis}

The functional evaluation of the 3D printed electrode structures in comparison to the $\mathrm{AgCl}$ electrodes is based on the classification accuracy. Table 4.2 shows the classification accuracy of each subject for each classifier. Confusion matrices show that in most cases, misclassifications were made with respect to the rest state (class 0). These misclassifications where both false positives and false negatives. Figure 4.17 shows the confusion matrix of the SVM classifier for the printed electrodes on subject 1 .

\subsection{Discussion}

\subsubsection{FDM Printing Process}

Some spots of the conductive TPU were seen in the dielectric part (Figure 4.2). These spots were caused by material oozing from the paused nozzle moving over 


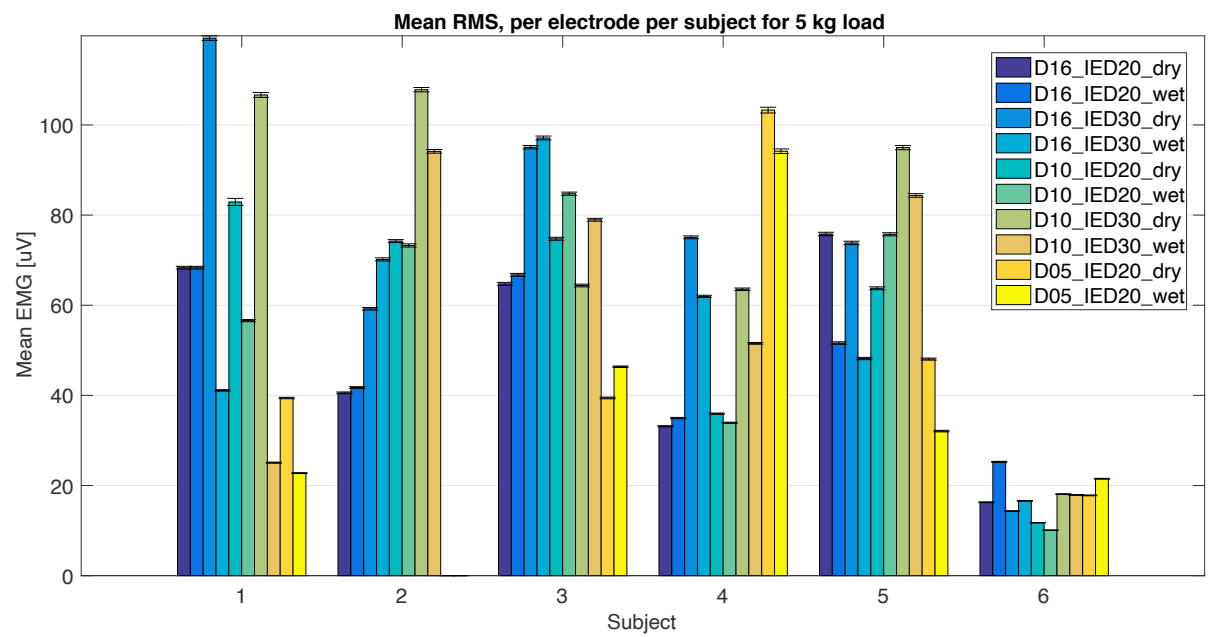

Figure 4.14: Mean envelope of the $5 \mathrm{~kg}$ load sample of each electrode size in wet and dry conditions, shown per subject.

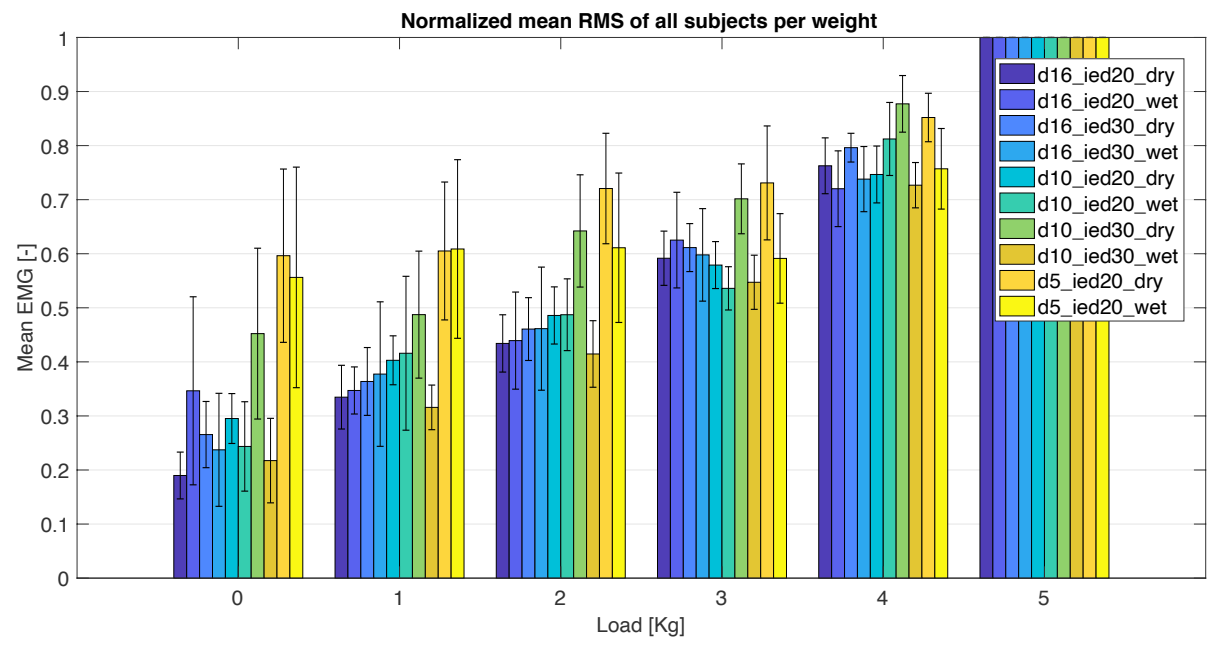

Figure 4.15: Mean envelope of each electrode obtained for all subjects, shown per load sample. 


\begin{tabular}{|c|c|c|c|c|c|c|}
\hline \multirow[b]{2}{*}{ d16_ied20_dry } & \multicolumn{6}{|c|}{ P-value between weight per electrode } \\
\hline & 0.01 & 0.01 & 0.01 & 0.01 & 0.01 & 0.01 \\
\hline \multirow{2}{*}{$\begin{array}{l}\text { d16_ied20_wet } \\
\text { d16_ied30_dry }\end{array}$} & 1 & 0.22 & 0.01 & 0.05 & 0.05 & 0.05 \\
\hline & 0.05 & 0.05 & 0.01 & 0.01 & 0.01 & 0.01 \\
\hline \multirow{4}{*}{ 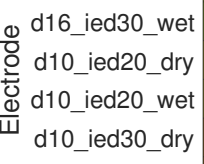 } & 0.05 & 0.1 & 0.05 & 0.1 & 0.01 & 0.01 \\
\hline & 0.1 & 0.21 & 0.01 & 0.05 & 0.01 & 0.01 \\
\hline & 0.1 & 0.49 & 0.18 & 0.01 & 0.05 & 0.01 \\
\hline & 0.45 & 0.01 & 0.3 & 0.01 & 0.1 & 0.05 \\
\hline \multirow{4}{*}{$\begin{array}{r}\text { d10_ied30_wet } \\
\text { d5_ied20_dry } \\
\text { d5_ied20_wet }\end{array}$} & 0.23 & 0.05 & 0.1 & 0.01 & 0.01 & 0.01 \\
\hline & 0.89 & 0.1 & 0.78 & 0.23 & 0.05 & 0.1 \\
\hline & 0.4 & 0.94 & 0.77 & 0.1 & 0.05 & 0.12 \\
\hline & $0-1$ & $1-2$ & $\begin{array}{l}2-3 \\
\text { ween }\end{array}$ & $\begin{array}{c}\text { 3-4 } \\
\text { ghts [ }\end{array}$ & $4-5$ & $0-5$ \\
\hline
\end{tabular}

Figure 4.16: Probability value of all subjects between different load samples for all tested electrodes sizes and conditions $(0.01,0.05$ and 0.1 indicate a $p$-value below the subsequent value).

Table 4.2: Classification accuracy of the linear discriminant analysis (LDA) and support vector machine (SVM) classifier.

\begin{tabular}{llllllll}
\hline & Subject: & $\mathbf{1}$ & $\mathbf{1}$ & $\mathbf{2}$ & $\mathbf{3}$ & & \\
\hline \multirow{2}{*}{ Classifier } & & \multirow{2}{*}{ AgCl } & ETPU & ETPU & ETPU & $\begin{array}{l}\text { Avg } \\
\text { ETPU }\end{array}$ & $\begin{array}{l}\text { Std } \\
\text { ETPU }\end{array}$ \\
\hline \multirow{2}{*}{ LDA } & & & & & & & \\
\multirow{2}{*}{ SVM } & 5 Class & $85.5 \%$ & $88.6 \%$ & $78.1 \%$ & $82.5 \%$ & $83.1 \%$ & $5.3 \%$ \\
& 3 Class & $81.6 \%$ & $86.5 \%$ & $77.5 \%$ & $80.2 \%$ & $81.4 \%$ & $4.6 \%$ \\
& 5 Class & $80.4 \%$ & $90.9 \%$ & $81.1 \%$ & $85.9 \%$ & $86.0 \%$ & $4.9 \%$ \\
\end{tabular}




\begin{tabular}{|c|c|c|c|c|c|c|c|}
\hline & \multicolumn{7}{|c|}{ Confusion Matrix } \\
\hline 0 & $\begin{array}{c}906 \\
40.1 \%\end{array}$ & $\begin{array}{c}\mathbf{2 7} \\
1.2 \%\end{array}$ & $\begin{array}{c}\mathbf{5} \\
0.2 \%\end{array}$ & $\begin{array}{c}17 \\
0.8 \%\end{array}$ & $\begin{array}{c}10 \\
0.4 \%\end{array}$ & $\begin{array}{c}6 \\
0.3 \%\end{array}$ & $\begin{array}{c}93.3 \% \\
6.7 \%\end{array}$ \\
\hline 1 & $\begin{array}{c}49 \\
2.2 \%\end{array}$ & $\begin{array}{c}212 \\
9.4 \%\end{array}$ & $\begin{array}{c}0 \\
0.0 \%\end{array}$ & $\begin{array}{c}0 \\
0.0 \%\end{array}$ & $\begin{array}{c}0 \\
0.0 \%\end{array}$ & $\begin{array}{c}0 \\
0.0 \%\end{array}$ & $\begin{array}{l}81.2 \% \\
18.8 \%\end{array}$ \\
\hline 2 & $\begin{array}{c}13 \\
0.6 \%\end{array}$ & $\begin{array}{c}0 \\
0.0 \%\end{array}$ & $\begin{array}{c}\mathbf{2 3 7} \\
10.5 \%\end{array}$ & $\begin{array}{c}0 \\
0.0 \%\end{array}$ & $\begin{array}{c}0 \\
0.0 \%\end{array}$ & $\begin{array}{c}2 \\
0.1 \%\end{array}$ & $\begin{array}{c}94.0 \% \\
6.0 \%\end{array}$ \\
\hline & $\begin{array}{c}30 \\
1.3 \%\end{array}$ & $\begin{array}{c}0 \\
0.0 \%\end{array}$ & $\begin{array}{c}0 \\
0.0 \%\end{array}$ & $\begin{array}{c}\mathbf{2 2 4} \\
9.9 \%\end{array}$ & $\begin{array}{c}0 \\
0.0 \%\end{array}$ & $\begin{array}{c}0 \\
0.0 \%\end{array}$ & $\begin{array}{l}88.2 \% \\
11.8 \%\end{array}$ \\
\hline 4 & $\begin{array}{c}32 \\
1.4 \%\end{array}$ & $\begin{array}{c}\mathbf{0} \\
0.0 \%\end{array}$ & $\begin{array}{c}0 \\
0.0 \%\end{array}$ & $\begin{array}{c}0 \\
0.0 \%\end{array}$ & $\begin{array}{c}\mathbf{2 3 3} \\
10.3 \%\end{array}$ & $\begin{array}{c}0 \\
0.0 \%\end{array}$ & $\begin{array}{l}87.9 \% \\
12.1 \%\end{array}$ \\
\hline 5 & $\begin{array}{c}22 \\
1.0 \%\end{array}$ & $\begin{array}{c}0 \\
0.0 \%\end{array}$ & $\begin{array}{c}0 \\
0.0 \%\end{array}$ & $\begin{array}{c}0 \\
0.0 \%\end{array}$ & $\begin{array}{c}0 \\
0.0 \%\end{array}$ & $\begin{array}{c}\mathbf{2 3 2} \\
10.3 \%\end{array}$ & $\begin{array}{c}91.3 \% \\
8.7 \%\end{array}$ \\
\hline & $\begin{array}{l}86.1 \% \\
13.9 \%\end{array}$ & $\begin{array}{l}88.7 \% \\
11.3 \%\end{array}$ & $\begin{array}{c}97.9 \% \\
2.1 \%\end{array}$ & $\begin{array}{c}92.9 \% \\
7.1 \%\end{array}$ & $\begin{array}{c}95.9 \% \\
4.1 \%\end{array}$ & $\begin{array}{c}96.7 \% \\
3.3 \%\end{array}$ & $\begin{array}{c}90.6 \% \\
9.4 \%\end{array}$ \\
\hline & 0 & 1 & 2 & 3 & $\star$ & $b$ & \\
\hline
\end{tabular}

Figure 4.17: Confusion matrix of subject one SVM classifier for the TPU electrodes. 
the structure together with the active nozzle. Oozing was kept to a minimum by fine-tuning the slicer setting for retraction and temperature. However, further improvements need to be made by improving the printer's mechanical design, such as incorporating retractable nozzles or adding the capability to park nozzles that are not in use. The second option gives the largest freedom in expanding the number of nozzles and materials.

Due to the nature of the FDM printing process, structures are built up from fields of parallel lines that are stacked onto each other. The contact interfaces between these lines and layers cause the conductivity of the printed structure to be anisotropic [233, 31], manly reducing the conductivity in the $z$-direction [31]. Further study of the anisotropy of the material will lead to further optimized printing patterns and electrode design.

\subsubsection{Contact Interface}

The four-point measurement (Figure 4.9) shows that the contribution of the medium is about 20 times lower than the electrode and contact interface resistance that are included in the three-point measurement of the printed electrode. The obtained impedance of the printed electrode in combination with the interface ranged from $7.8 \mathrm{k} \Omega$ at $20 \mathrm{~Hz}$ to $4.3 \mathrm{k} \Omega$ at $250 \mathrm{~Hz}$, and the impedance of the $\mathrm{AgCl}$ ranged from $650 \Omega$ to $600 \Omega$. These values are within the range of carbonloading silicone rubber electrodes and $\mathrm{AgCl}$ electrodes reported by Webster et al. [207]. Due to the high input impedance (over $100 \mathrm{M} \Omega$ ) of the used biopotential amplifier, the relatively high electrode electrolyte interface of the printed electrode should not influence the signal quality. However, it is likely that the electrode-to-skin interface impedance in dry circumstances is higher than in the situation tested with the electrolyte solution. Furthermore, the skin's epidermis and subcutaneous layers will increase the electrode-to-skin impedance as well [207].

\subsubsection{Electrode Evaluation}

The results of the evaluation between the printed and the $\mathrm{AgCl}$ electrodes showed that both electrodes are capable of detecting various levels of muscle activity (Figure 4.13). In Figure 4.12, five out of six subjects showed a lower amplitude in the mean envelope of the TPU electrodes compared to the $\mathrm{AgCl}$ electrodes. However, in this setup it is not possible to validate whether the performance of the $\mathrm{AgCl}$ electrodes is better than the printed electrodes due to the fact that the electrodes are placed side-by-side above the biceps brachii; therefore, the electrodes did not measure the exact same volume. 
In the electrode size comparison study, the printed electrodes were swapped sequentially after each measurement with a different type varying in diameter and/or inter-electrode distance. Care was taken to place each electrode at the same location as the previous electrode; however, exact repeatable placement was hard to accomplish. Furthermore, there might be a difference between the subjects in subcutaneous fat layers and muscle size, leading to the large individual differences shown in Figure 4.14. Therefore, this figure does not show a clear relation between electrode size and condition. However, the results strongly suggest that larger electrodes have a performance advantage over smaller electrodes, since the smaller electrodes were less able to detect an amplitude change caused by an increase in load and electrodes with a diameter of $5 \mathrm{~mm}$ were unable to obtain a relevant EMG signal in some individuals. Although the performance difference between the wet or dry conditions was not evident in this study, further research on the long-term performance could show a performance increase of the dry electrodes due to moisture build-up [216, 217] and a decrease of wet electrode performance due to evaporation [216, 218, 214].

\subsubsection{Hand Posture Classification}

The intention of the classifiers is to show the possibility of using 3D printed electrodes structures to perform online control of prosthetic devices and to compare the $3 \mathrm{D}$ printed electrodes against the $\mathrm{AgCl}$ electrodes. Through the results, we were able to prove that the classification accuracy of signal recorded using TPU electrodes is of the same level as the accuracy of the conventional $\mathrm{AgCl}$ electrodes. The accuracy came close to values of comparable structures reported by Daley et al. [234] and Li et al. [235]. Moreover, these values can even be improved by looking at the confusion matrices and understand the underlying flaws of the classifier and improving them by going back to the training and rebuilding the classifier. Moreover, the application of more advanced algorithms, such as neural networks, may further improve the classification performance. These algorithms could also facilitate the use of smaller electrodes. The smallest electrodes tested in this study, with a diameter of $5 \mathrm{~mm}$, were capable of measuring EMG signals in some individuals but were unable to resolve the small changes in muscle activity. This information could still be of use in, for example, (high density) grid structures, where the information of multiple sensors could be combined by smart algorithms.

The tested structures presented in this work are relatively simple and flat. However, 3D printing allows for the fast creation of far more complex structures that could be personalized to the user. A more complex, proof of principle 3D 

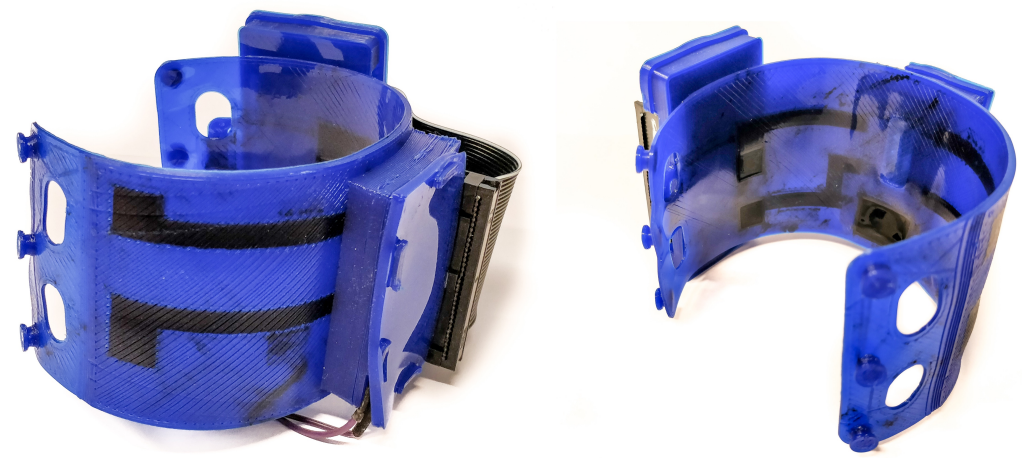

Figure 4.18: Proof of concept custom-sized 3D printed EMG armband. The electrodes are internally routed to an EMG amplifier fitted in a housing integrated in the band.

printed five electrode EMG band is shown in Figure 4.18; the size and electrode placement of this armband are made specific to one user. Furthermore, the armband houses a small amplifier and microcontroller that could wirelessly transmit the data. These 3D printed structures require minimal post-production steps and are easily scalable in terms of both the size and number of electrodes, which makes this technique highly accessible. Complex wire routing and shielding can be done in three dimensions inside of the structures, greatly reducing the amount of wiring needed. In the future, sEMG electrodes might be integrated into assistive devices and prostheses that are directly $3 \mathrm{D}$ printed without the need for additional post-processing steps. This allows for easy donning and doffing of the assistive device due to the customized electrode positions and multiple electrodes. Furthermore, the 3D printed dry electrodes are better-suited for long-time and multiple uses in prostheses since the wet $\mathrm{AgCl}$ electrodes can only be used once and will dry out over time.

\subsection{Conclusions}

This work shows the potential use of additive manufacturing techniques for the creation of flexible personalized sEMG sensing structures. These structures have been successfully printed on a low-cost consumer-grade multi-material FDM printer modified to extrude flexible TPU materials. The material cost of one sensing structure, containing two electrodes, is only $€ 0.20$. Comparison between the gold standard $\mathrm{AgCl}$ gel electrodes and the printed EMG electrodes 
with comparable contact areas showed that there is no significant difference in the EMG signal envelope values. This makes 3D printed electrodes based on conductive TPU highly suitable for practical applications.

The results suggest that $3 \mathrm{D}$ printed electrodes with a size comparable to the gold standard electrodes have a performance advantage compared to smaller 3D printed electrodes. Large inter-individual differences in the recorded electrode amplitudes were observed both within and between subjects. Even the smallest electrodes, with a diameter of $5 \mathrm{~mm}$, were capable of measuring EMG signals in some individuals. However, these electrodes were shown to be unable to resolve small changes in muscle activity. Overall, this work has shown that $3 \mathrm{D}$ printed electrodes, made from a combination of carbon-doped and regular TPU, display a performance that is largely comparable to the gold-standard $\mathrm{AgCl}$ electrodes when paired with the high-quality amplifiers used in this research. 


\title{
Chapter 5
}

\section{A 3D printed soft fingertip sensor for measur- ing normal and shear components of interac- tion forces}

\begin{abstract}
Sensing of the interaction forces at fingertips is of great value in assessment and rehabilitation therapy. Current force sensors are not compliant to the fingertip tissue and result in loss of touch sensation of the user. This chapter shows the development and characterization of a flexible fully-3D printed piezoresistive shear and normal force sensor that uses the mechanical deformation of the finger tissue. Two prototypes of the sensing structure are evaluated using a finite element model and a measurement setup that applies normal and shear forces up to $10 \mathrm{~N}$ on a fingertip phantom placed inside the sensing structure, which is fixed to prevent slippage. Furthermore, the relation between strain (rate) and resistance of the conductive TPU, used for the strain gauges, is characterized. The applied normal and shear force components of the 3D printed sensing structure can be partly separated. FEM analysis showed that the output of the sensor is largely related to the sensor geometry and location of the strain gauges. Furthermore, the conductive TPU that was used has a negative gauge factor for the strain range used in this study and might cause nonlinear behaviors in the sensor output.
\end{abstract}

This chapter is published as: G. Wolterink, R. Sanders, B.J. van Beijnum, P. Veltink, G. Krijnen, A 3D printed Soft Fingertip Sensor for Providing Information about Normal and Shear Components of Interaction Forces, Sensors 21 (13), 4271, 2021 


\subsection{Introduction}

Measurement of the interaction forces between the fingertips and the external environment is of great value in upper extremity assessment and for interaction in rehabilitation therapy where patients need to relearn motor tasks [236]. While handling objects, these interaction forces can reach up to $50 \mathrm{~N}$ for normal and up to $10 \mathrm{~N}$ for shear forces, where the high dynamic range for resolving both forces is below $10 \mathrm{~N}[14,237]$. Current available force sensors, capable of measuring shear and/or normal forces, show two main difficulties. Most sensors are mainly made of stiff materials leading to the loss of touch sensation of the user. Secondly, these sensors are not ideally adapted to the shape and stiffness of the fingertip, leading to poor sensor-to-skin attachment. Due to this poor contact the sensors tend to shift easily by external forces [18]. In practice this issue has presented itself in the form of limited usefulness and reliability of current force sensors [19].

In recent years the interest in and development of 3D printed sensors have increased due to the advent of multi-material printers and increasing availability of conductive thermoplastic materials [81, 28, 124]. The introduction of soft and flexible materials with electrical properties creates the opportunity for applications in soft robotics [96]. Furthermore, the high customizability of 3D printing makes these sensors an ultimate candidate for biomedical applications such as biopotential electrodes [93, 90], tactile and bending sensors [94, 87], and soft finger shear- and normal-force sensors [91]. Ideally, the sensor on the fingers should be soft and not affect the touch sensation of the user. These 3D printed sensor structures have potential advantages for development of complex sensing structures that cannot be produced using traditional production methods, while still being highly customizable to fit to an individual user. Furthermore, these sensing structures may in the future be integrated into larger, multi-functional structures [28].

The goal of this work is to create and characterize a degree of freedom (DoF) force sensor, which measures the normal and interaction shear forces between the environment and the fingertips. To minimize the loss of touch sensation of the user, the sensor structure should be compliant with the soft finger tissue and ensure direct interaction between fingertip skin and the object.

\subsection{Design}

The sensing principle of the sensor is based on the mechanical deformation of soft tissue around the bone (distal phalanx) in the fingertip [91]. A normal or shear force applied by the fingertips results in a movement and compression of 

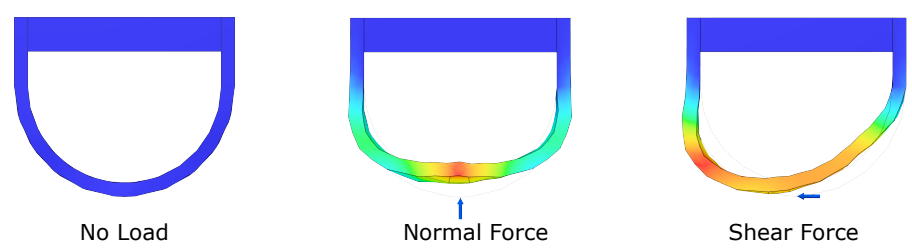

Figure 5.1: Deformation of the sensing structure under normal and shear force, colors represent amount of deformation.
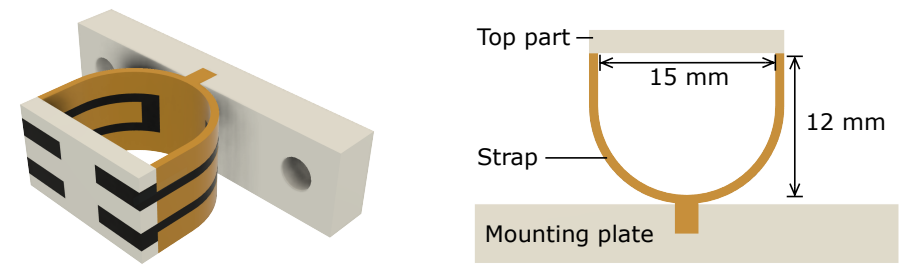

Figure 5.2: Render of the sensor design. Orange represents non-conductive TPU material, black represents the strain gauges made of conductive TPU material, and the rigid PETG parts are represented in gray.

the soft tissue relative to the distal phalanx and nail-bed. This movement can be sensorized by placing a flexible strap, with embedded strain gauges, on top of the nail and around the fingertip. Since a normal force exerted on the fingertip results in a symmetric deformation on both sides of the straps and a shear force induces a non-symmetric deformation (see Figure 5.1), the strap contains two strain gauges, one on each side, to separate normal from shear force.

Yin et al. [237] demonstrated a comparable setup using traditional fabrication methods using microfluidic channels filled with liquid metal. Their design focuses on robotic tactile sensing and therefore does not focus on facilitating the touch sensation of human fingertips, and the design and characterization lack the presence of the distal phalanx and nail-bed. Nevertheless, the work by Yin et al. supports the interest in these types of sensors in (soft) robotic tactile sensing or use in prosthesis for haptic feedback applications.

The design and dimensions of the sensors are shown in Figure 5.2. The flexible strain sensors are made from carbon-doped conductive TPU (PI-ETPU, Palmiga Innovation, Jonstorp, Sweden) and are represented by the black-colored material. The orange flexible strap is made from non-conductive TPU material (NinjaFlex, Fenner Drives, Manheim, PA, USA) and has a width of $0.8 \mathrm{~mm}$, being two traxels (track-elements).

To study the influence of the strain gauge location, two versions of the sensor 
were designed. One structure has the strain gauges placed on the inside (inside sensor), having only non-conductive material on the outside, while the second sensor has the opposite design with the strain gauges implemented on the outside (outside sensor).

The rigid top part and bottom mounting plate of the sensor are made from PETG (3DJake, Niceshops GmbH, Paldau, Austria) and are represented in gray. The bottom mounting plate can be mounted onto the test setup and is fixed to the center-line of the flexible strap of the finger sensor, allowing for shear force measurements without slip.

\subsection{Numerical Simulation}

A finite element method (FEM) simulation was performed using a Static Structural Analysis in Ansys Workbench (ANSYS Inc., Canonsburg, PA, USA) to estimate the strain induced by normal and shear forces on the inside and outside of the sensor's geometry. A cross-section of the finger model including the sensor is shown in Figure 5.3. To simulate the relatively low Poisson's ratio of the soft finger tissue [238], the soft tissue part of the finger phantom is made from TPU with a $30 \%$ honeycomb infill. Both the conductive and non-conductive TPU materials were modeled using the five-parameter Mooney-Rivlin hyperelastic material model that was fitted on experimental stress-strain data of the NinjaFlex TPU performed by Twank et al. [239]. The other materials were selected from the Ansys Engineering data library. The mechanical properties of the "finger bone" were modeled as stainless steel (316, annealed) due to the high stiffness of bone (17 GPa [238]) and to the chosen stainless steel fixture in the test setup; other stiff parts were modeled as PET (amorphous). Both the situation where only a normal force and the situation where only a shear force is applied were modeled by applying a $10 \mathrm{~N}$ sinusoidal remote force on the finger bone with a frequency of $0.5 \mathrm{~Hz}$.

To estimate the strain and corresponding resistance change, a path along the curve of the strain gauge is taken. This path is subsequently divided into elements for which the directional changes in the $x$ - and $y$-directions $(d x$ and $d y)$ are computed. Since there are two versions of the sensor, one path is taken along the inside surface of the strap and the other along the outside surface of the strap. These directional changes per element in the $x$ - and $y$-directions are exported to Matlab (Mathworks Inc., Natick MA, USA) to calculate an 


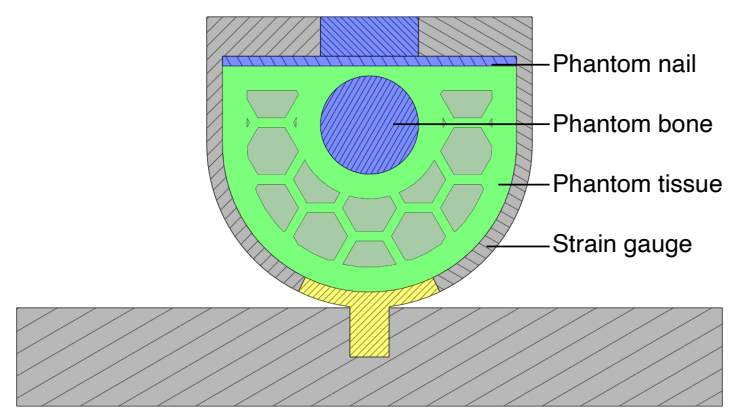

Figure 5.3: Cross-sectional view of finger sensor with phantom, printed with $30 \%$ honeycomb infill.

estimation of the strain of the $i$ th element $\left(\varepsilon_{i}\right)$ :

$$
\varepsilon_{i}=\frac{1}{L_{i 0}} \sqrt{\left(d x_{i+1}-d x_{i}\right)^{2}+\left(d y_{i+1}-d y_{i}\right)^{2}}
$$

where $L_{i 0}$ is the initial length of element $i$.

The results of Equation (5.1) in the case of the outside path are visualized in Figure 5.4, showing the strain per element as a function of time.

Next, the resistance change of the whole path $(\Delta R / R)$ can be computed by multiplying the elemental strain with the gauge factor $(G F)$ and initial resistance at zero load per element $\left(R_{0 i}\right)$ :

$$
\frac{\Delta R}{R}=\frac{1}{R_{0}} \sum_{i=1}^{n-1} \varepsilon_{i} G F R_{0 i}
$$

The sum of the elemental resistance of the path $\left(R_{0}\right)$ is set to $2.2 \mathrm{k} \Omega$ and a gauge factor $(G F)$ to -15 for ETPU. Because the U-shaped strain gauges are symmetrical, only the path of one side of the loop is taken. For simplicity, it is assumed the connection pads and bottom loop only contribute as fixed mutual resistance.

\subsection{Methods}

Both the inside and outside sensor designs were evaluated using a linear actuator that applies forces to a 3D printed phantom fingertip with the same design and materials as discussed in Section 5.2. Furthermore, the relation between the resistance and the strain rate of the conductive TPU were studied. 


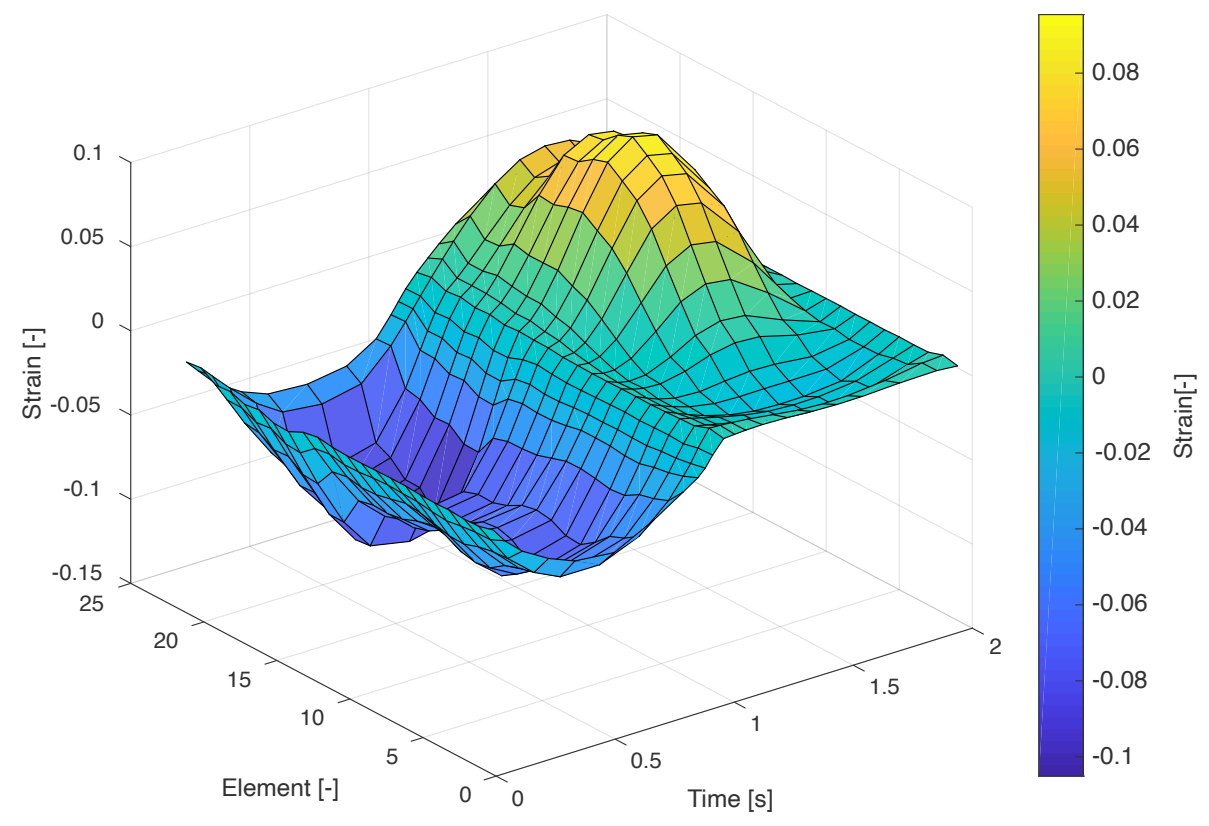

Figure 5.4: Estimated elemental strain as a function of time, per element of the outside path (only the path of one side of the strain gauge loop is taken). A $0.5 \mathrm{~Hz}$ sinusoidal shear force of $10 \mathrm{~N}$ was applied in the simulations. 


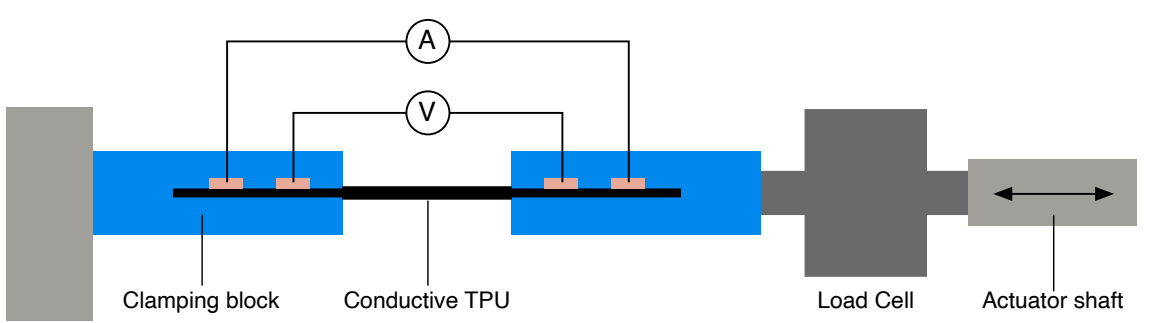

Figure 5.5: Setup to measure the resistance while straining a sample of conductive TPU. A constant current was applied on the sample while it was stretched at a constant rate using a linear actuator.

\subsubsection{Characterization of Conductive TPU}

To investigate the influence of strain on the resistance of the conductive TPU filament used in the sensor model, a sample conductive TPU filament (ETPU 85-700+, Palmiga Innovation, Jonstorp, Sweden) with a diameter of $1.75 \mathrm{~mm}$ was loaded into a strain setup. In this setup the filament was clamped between two blocks containing copper strips to connect the sample in four point configurations to determine the resistance as shown in Figure 5.5. The distance between the two clamping points was $10 \mathrm{~mm}$.

A current of $100 \mu \mathrm{A}$ was supplied by a source measure unit (Model 2410, Keithley Instruments, Inc., Cleveland, OH, USA) and the voltage drop was recorded using a USB oscilloscope (Handyscope HS5, TiePie engineering, Sneek, The Netherlands). One side of the clamping blocks was fixed while the other side was pulled along the length direction of the sample using a linear actuator (SMAC LCA25-050-15F, SMAC, Carlsbad, CA, USA) position controlled by a computer using Matlab (Mathworks Inc., Natick, MA, USA). The force was measured by a load cell (LCMFD-50N, Omega Engineering, Norwalk, CT, USA) placed between the actuator and the clamping block. The output of the load cell was amplified (IAA100, Futek, Irvine, CA, USA) and connected to the USB oscilloscope together with the encoder output of the linear actuator to synchronize with the resistance change.

The samples were strained at a strain rate of $1 \varepsilon / \mathrm{min}$ until a length of about $140 \%$, after which the sample was unloaded at the same rate. After 10 cycles the sample of conductive TPU filament was replaced with a fresh sample and the experiment was repeated at strain rates of 5,10 , and $20 \varepsilon / \mathrm{min}$. 

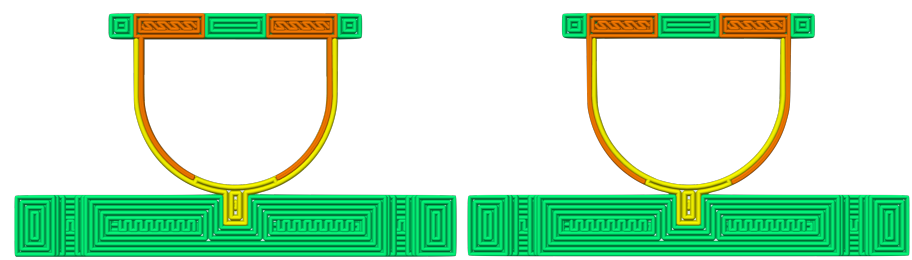

Figure 5.6: Top view of printing pattern of the inside (left) and outside (right) sensors. The conductive TPU is shown in orange, non-conductive TPU in yellow, and PETG in green.

\subsubsection{Sensor Fabrication}

The strap of the sensors has a thickness of two traxels. Two versions of the sensor were printed, the first having the strain gauges positioned on the inside traxels of the strap (inside sensor); the second has the strain gauges positioned on the outside (outside sensor) as shown in Figure 5.6. The sensor was printed in one go using a Diabase H-Series Hybrid (Diabase Engineering, Longmont, CO, USA) multi-material FDM 3D printer. Figure 5.7 shows a photograph of the printed inside sensor. Control of the printer and printer settings was handled by the slicer software (Simplify3D, Inc., Cincinnati,OH, USA). The layer height of the structure was set to $200 \mu \mathrm{m}$. After printing, the sensor was annealed for 15 hours at $80^{\circ} \mathrm{C}$. Electrical interfacing to the conductive TPU of the sensor was made by melting a fine stranded copper wire into the connection pads of the sensor. The initial resistance of the strain gauges measured at this interface was in the range of $5 \mathrm{k} \Omega$.

\subsubsection{Sensor Characterisation}

The finger phantom was built from a low infill (30\%) honeycomb structure made of TPU, representing the soft tissue, printed in one go, together with a $1 \mathrm{~mm}$ thick PETG part, representing the nail. The finger bone is represented by a stainless steel bolt with a diameter of $5 \mathrm{~mm}$.

The force was applied to the stainless steel bolt by a mounting bracket connected to the shaft of the linear actuator (SMAC LCA25-050-15F, SMAC, Carlsbad, CA, USA). To apply both normal and shear force components to the phantom the actuator can rotate, with its center of rotation around the bolt through the phantom, from $-90^{\circ}$ to $90^{\circ}$, where only a shear force is applied and at $0^{\circ}$ only a normal force is applied. The force applied by the linear actuator was measured by a load-cell in line with the actuator shaft (LCMFD-50N, Omega Engineering, Norwalk, CT, USA). The setup is shown in Figure 5.8. 


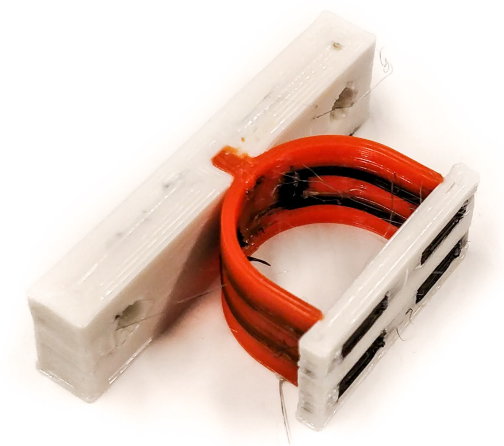

Figure 5.7: Photograph of the printed sensor with the strain gauges positioned on the inside. The conductive TPU parts are shown in black, non-conductive TPU in orange, and the PETG parts in white.

The resistance change of the strain gauges was read out using a Wheatstone bridge configuration. Each strain gauge was connected separately in a quarter bridge configuration, the applied bridge voltage was $5 \mathrm{~V}$ and the output of the bridge was measured using an instrumentation amplifier (AD620, Analog Devices, Norwood, MA, USA). The amplified signal, representing the resistance change of each strain gauge, was logged together with the encoder output of the linear actuator and the output of the load-cell amplifier (IAA100, Futek, Irvine, CA, USA) using a digital oscilloscope (Handyscope HS5, TiePie engineering, Sneek, the Netherlands).

The sensor was actuated under multiple angles to apply both shear and normal force components. At $0^{\circ}$ the only force component is the normal force and at $\pm 90^{\circ}$ there is only a shear force component. Under each angle the actuator produces a sinusoidal force of $0.5 \mathrm{~Hz}$ with an amplitude of $10 \mathrm{~N}$ for $20 \mathrm{~s}$. The oscilloscope data were sampled at $200 \mathrm{kHz}$ to capture all the encoder pulses and downsampled by a factor 10 for further analysis. Next the data from the load-cell and finger sensor strain gauges were low-pass-filtered using a $40 \mathrm{~Hz}$ second-order Butterworth filter to remove any high-frequency noise from the data. The finger sensor data were also passed through a $0.5 \mathrm{~Hz}$ second-order Butterworth high-pass filter to remove the drift. Next the normalized sensor output was obtained $(\Delta S)$ by subtracting the mean of the absolute value of the whole dataset of that sensor $\left(\Delta R_{\text {mean }}\right)$ from the measured resistance change 

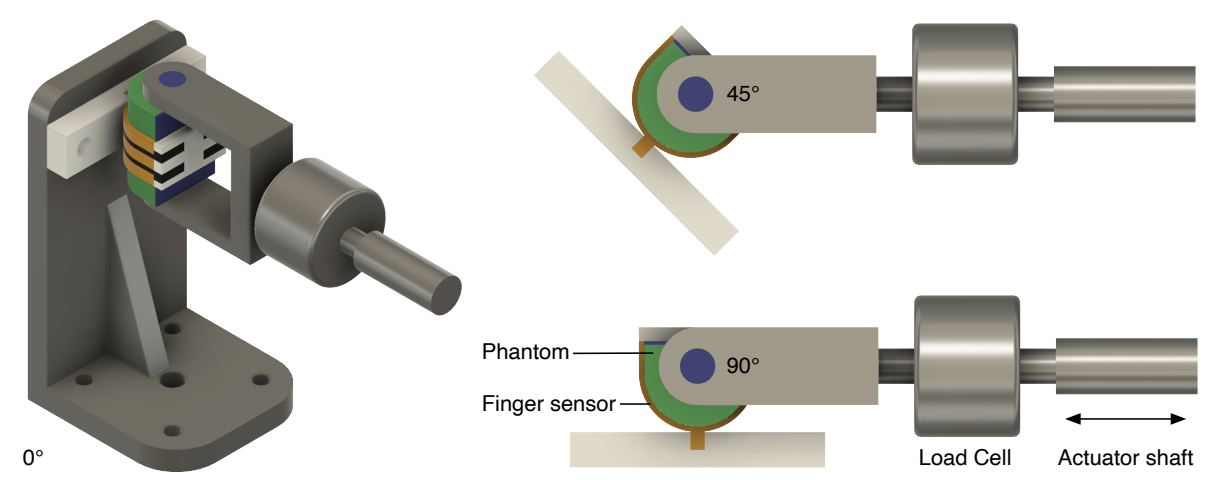

Figure 5.8: Measurement setup: the finger sensor and phantom can rotate around the phantom bone allowing the linear actuator to apply both normal and shear force components.

$\left(\Delta R_{\text {meas }}\right)$ and subsequently dividing the result by $\Delta R_{\text {mean }}$ (see Equation (5.3)).

$$
\Delta S=\frac{\Delta R_{\text {meas }}-\Delta R_{\text {mean }}}{\Delta R_{\text {mean }}}
$$

Next the loading cycles were segmented and the mean and standard deviation from the force, position, and sensor output $(\Delta S)$ of these cycles were taken. To exclude potential unwanted discrepancies at the start and stop of the measurements, the first and last cycles were excluded from the average data.

\subsection{Results}

\subsubsection{Characterization of Conductive TPU}

Figure 5.9 shows the average relative resistance change over eight cycles (second till second-last cycle) for several strain rates. The figure shows for each strain rate a decline in resistances up to a strain of around 0.2, after this point the resistance slowly increases with increasing strain.

Figure 5.10 shows the recording of one sample that is strained ten times at a strain rate of $1 \varepsilon / \mathrm{min}$. The graphs clearly show the difference in the first cycle compared to the subsequent cycles. Furthermore, Figure 5.10 shows, in the bottom-right plot, that after the cyclic straining has been stopped and the material is back to the original position, the resistance is still clearly decreasing. 


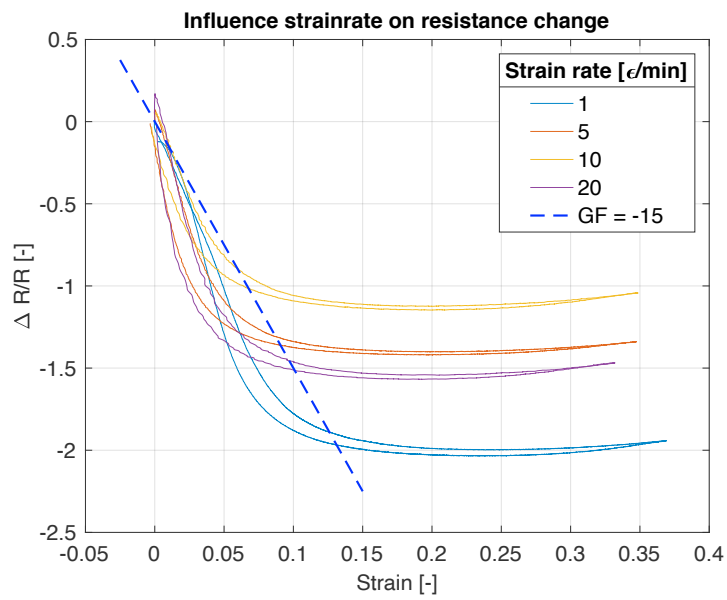

Figure 5.9: Average relative resistance change $(\Delta R / R)$ at various strain rates for ETPU filament: $\Delta R$ is given by the difference between initial resistance $\left(R_{0}\right)$ and the measured resistance $(R)$. The blue dashed line indicates the resistance change with a constant gauge factor $(\mathrm{GF})$ of -15 .
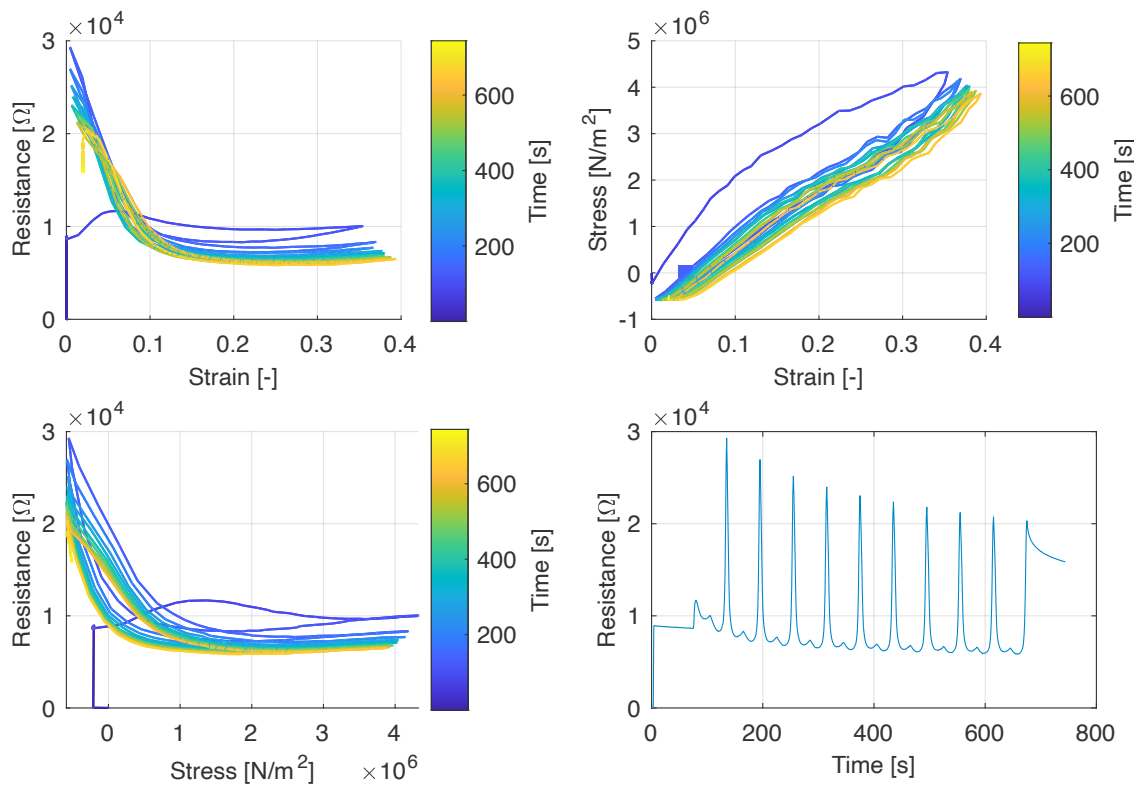

Figure 5.10: Top left: Resistance as a function of strain and time. Top right: Stress (initial diameter)-strain curve. Bottom left: Resistance as a function of stress and time. Bottom right: Resistance change over time during and $70 \mathrm{~s}$ after the measurement. These figures show all 10 strain cycles at a strain rate of $1 \varepsilon / \mathrm{min}$. 

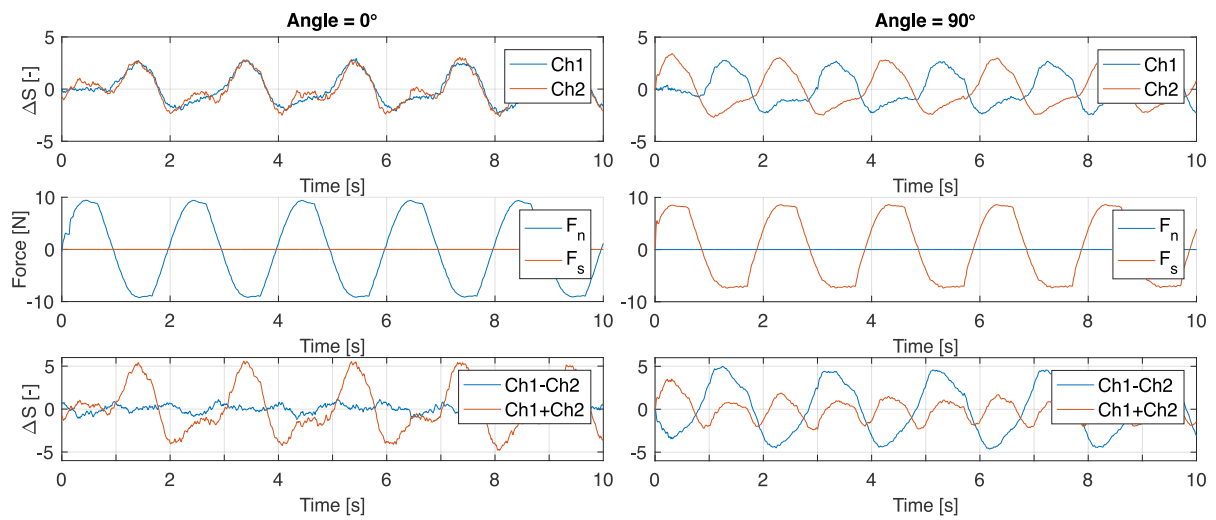

Figure 5.11: Time domain data of outside sensor for the first five force cycles where only a normal force $\left(0^{\circ}\right)$ and shear force $\left(90^{\circ}\right)$ are applied.

\subsubsection{Force Sensor}

Figure 5.11 shows the time domain data for the outside sensors of the first five force cycles in the two extreme positions $\left(0^{\circ}\right.$ and $\left.90^{\circ}\right)$. At $0^{\circ}$ angle it is clearly visible that both strain gauges have very similar signal outputs, whereas at an angle of $90^{\circ}$ the outputs of the two strain gauges are shifted by half a period. The standard deviation in Figure 5.12 shows that these cycles are repeatable. Furthermore, at $0^{\circ}$, it shows that the sensor is more sensitive to tension than to compression.

The combined results for the average sensor response and the modeled response of both the inside and outside sensors are shown in Figure 5.13. For the normal forces Figures 5.13a, b show that the slope of the estimated resistance change from the FEM model is close to the recorded sensor output, mainly in situations where the strain-gauge is under tension. For shear force loading, Figures $5.13 \mathrm{c}, \mathrm{d}$, the shape and slope of the model, come close to the measured data in both negative and positive shear, especially for the outside sensor. Figure 5.14 shows the sum and difference of sensors one and two of the averaged data for the outside sensor. The Figure shows that the difference (ch1-ch2) between the two sensors is not very sensitive to a normal force but clearly shows a stronger dependence on shear force. The relation of the sensor output sum and difference against normal force $\left(0^{\circ}\right)$ and shear force $\left(90^{\circ}\right)$ are shown in Figure 5.15.

The fitted data indicated in green in Figure 5.15 were used to estimate the sensor output in the $45^{\circ}$ and $-45^{\circ}$ situation where the normal and shear force components are equal. These estimated data against the measured sensor 

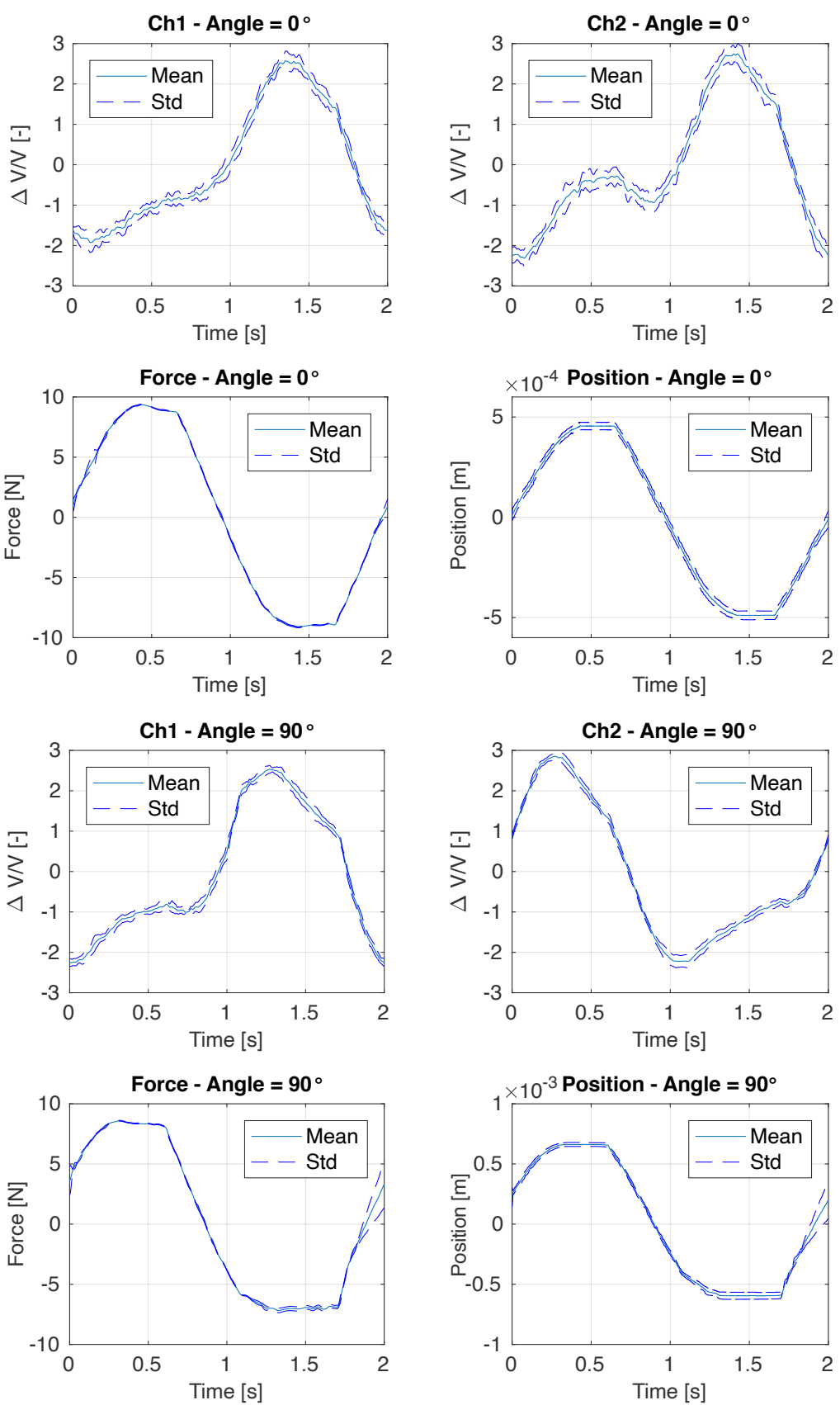

Figure 5.12: Averaged time domain data and standard deviation of the second until the ninth cycle where only a normal force $\left(0^{\circ}\right)$ and shear force $\left(90^{\circ}\right)$ are applied to the outside sensor. The position represents the position of the actuator shaft. 


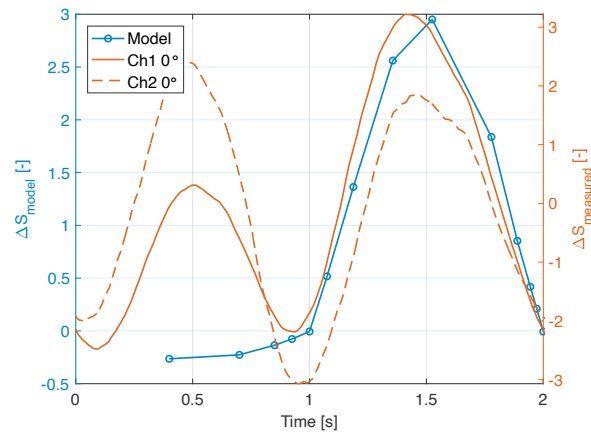

(a)

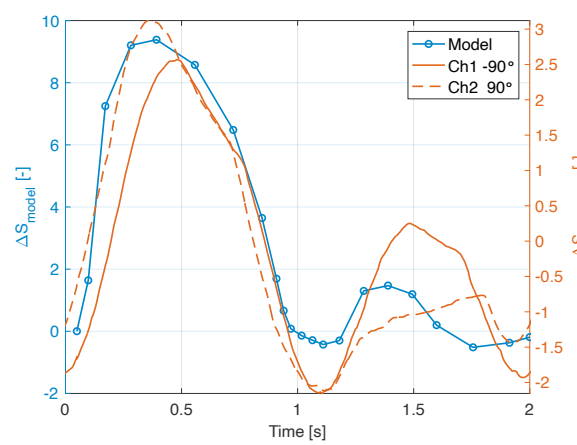

(c)

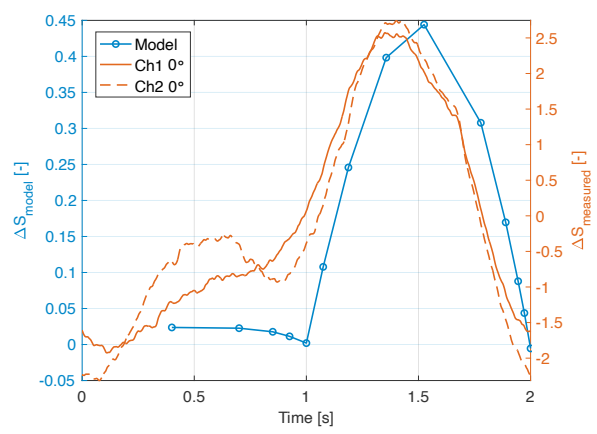

(b)

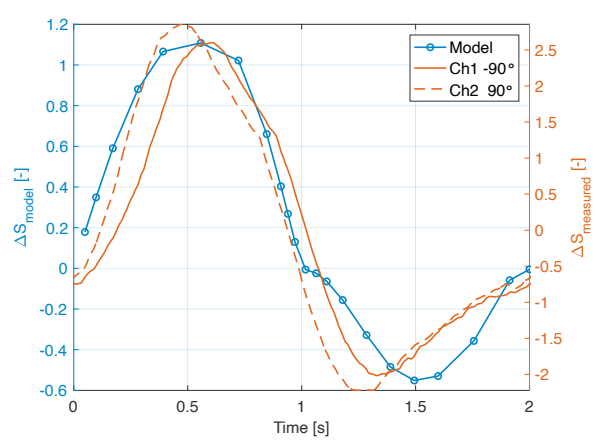

(d)

Figure 5.13: Average sensor response and the modeled (FEM) response of both the inside and outside sensors. (a) Normal force inside sensor; (b) normal force outside sensor; (c) shear force inside sensor; (d) shear force outside sensor.

output are shown in Figure 5.16. As indicated before, these data follow the applied shear force but in the normal force situation are limited to primarily sensing the tension force. 

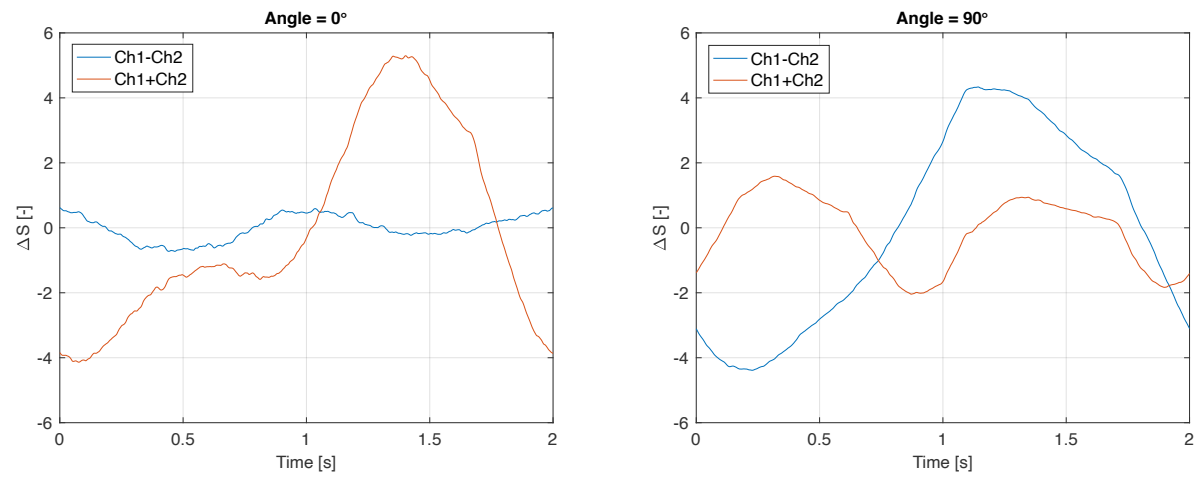

Figure 5.14: Sum and difference of the two strain gauges of the outside sensor in normal and shear situations.
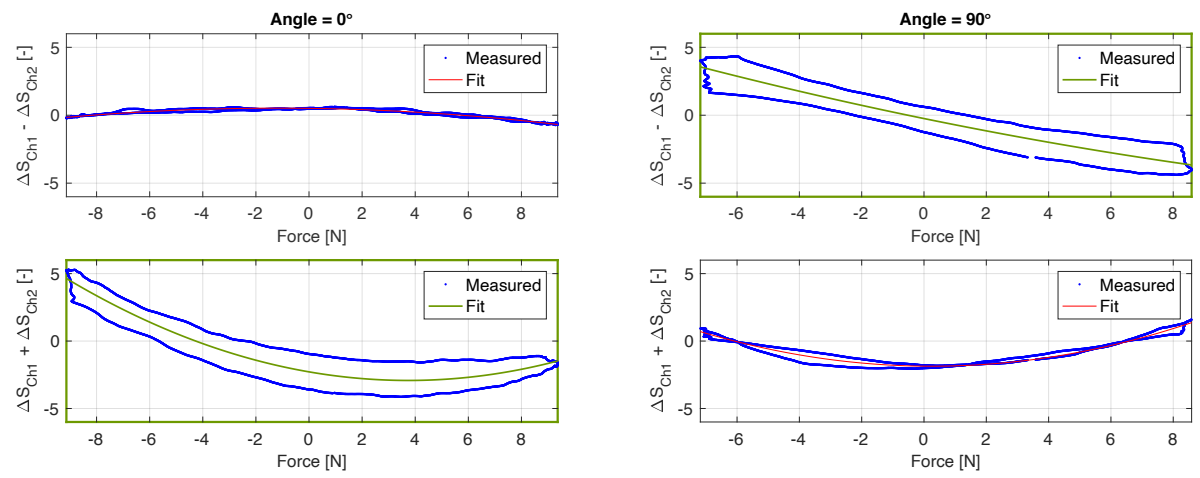

Figure 5.15: Relation of the outside sensor output sum and difference against normal force $\left(0^{\circ}\right)$ and shear force $\left(90^{\circ}\right)$. The fitted data indicated in green were used to estimate the normal (Fn) and shear force (Fs) components. 

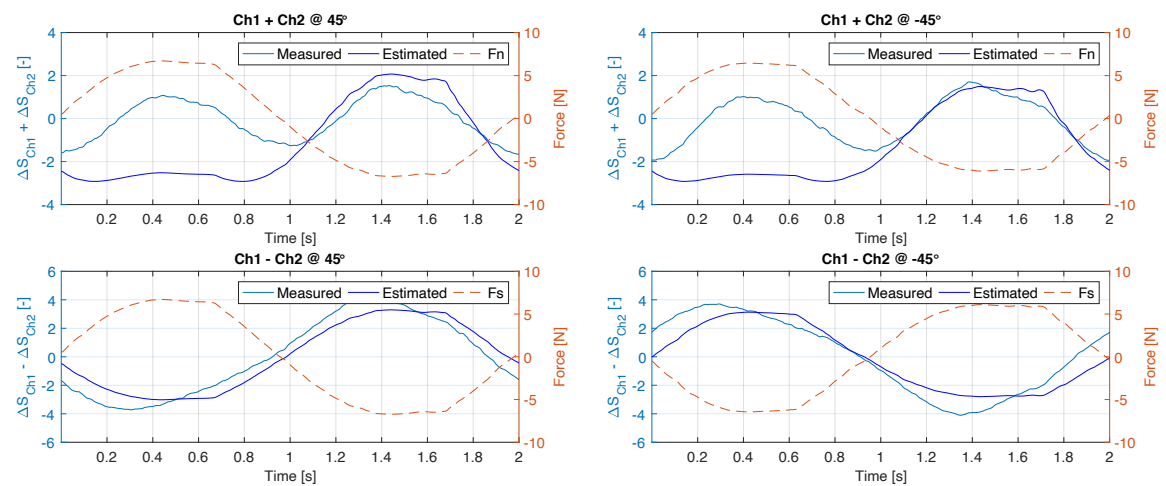

Figure 5.16: Estimated sensor output using the fit indicated in Figure 5.15 and the measured sensor output at $45^{\circ}$ and $-45^{\circ}$ of the outside sensor.

\subsection{Discussion}

\subsubsection{Characterization of Conductive TPU}

The results in Figure 5.10 clearly show that the first strain cycle deviates from the following cycles. This effect, shown in the stress-strain curve, is known as softening behavior [151], where in this case most softening happens in the first cycle. Furthermore, the conductive TPU materials seem to have a settling time when going back to the initial resistance at zero strain. This settling time is visible in the bottom-right graph of Figure 5.10 after $675 \mathrm{~s}$, where after the last cycle the resistance of the material slowly decreases. This behavior is not uncommon for conductive polymer composite materials [240, 124] and corresponds to the time-dependence stress-strain curves for TPU materials [151]. We note that the initial resistance drop at low strain values may be related to alignment of conductive carbon black particles in the TPU material, whereas at higher strains breaking down of these networks may be causing the increase in resistance, as proposed in [124]. Additionally, Rusinek et al. hypothesize the formation of new conductive pathways, caused by the contraction of the sample transverse to the strain due to Poisson's ratio [241]. Furthermore, it is believed that the mobility of polymer chains causes the formation and breakdown of conductive networks [149], where at higher strain rates the resistance will increase more by this effect compared to lower strain rates since the breakdown effect is more dominant at high strain rates $[149,150]$. Although Figure 5.9 does not directly show this trend, there is a relatively large difference between $1 \varepsilon / \mathrm{min}$ 
and the faster strain rates.

Christ et al. [124] have found the piezoresistive gauge factors to be similar for bulk material and FDM 3D printed samples. However, care has to be taken in the sensor design since the bulk material resistivity does not directly relate to the resistivity of a printed volume. Due to the nature of the FDM 3D printing process the structures are built of track elements (traxels) that are stacked in layers. The contact interfaces between these traxels and layers result in anisotropic structures with the largest resistance in the direction of the stacked layers (z-direction) [30, 31]. These effects should be taken into consideration while designing the printing pattern of conductive structures.

\subsubsection{Force Sensor}

Under loading of the normal force the strain gauges are bent at the edge and are not ideally compressed, making them less sensitive for compressive loading compared to tension forces where the sensors are strained. This is also expected on the basis of the FEM simulations. However, the actual sensors show higher sensitivity, especially in the inside configuration, than the simulated ones. The estimated output shown in Figure 5.16 also shows the lack of proper positive normal force estimation. Furthermore, the "parabolic" shape of the normal force $\left(F_{\mathrm{n}}\right)$ as a function of the sum of the sensor output shown in Figure 5.15 limits the possibility in an inverse model to distinguish between compression and tension forces. In a practical situation, where the sensor is placed on the fingertips to capture interaction forces, this might not be an issue since there are no tension force in the normal direction of the fingertips.

The output of the left and right sides of the strain gauges (Ch1 and Ch2 in Figure 5.11) should in theory be exactly similar due to the symmetry in the sensor design and measurement setup. However, experimental characterization shows some discrepancies between the two sensors. Besides possible inaccuracies in the measurement setup, we believe that the main cause is in the fabrication of the sensor due to inaccuracies in material extrusion during the printing process, material inhomogeneities, fluctuations in filament diameter, and variations in the bonding between traxels and layers.

The estimated resistance change by the FEM model is currently based on a constant negative gauge factor. However, the gauge factor of the ETPU material varies with the applied strain and strain rate as indicated by Figure 5.9. The FEM models of the strain gauge structures show the minimal and maximal strain per element to be -0.64 and 2.11 at the inside and -0.07 and 0.09 at the outside for the normal force situation. In the shear force situations the minimal 
and maximal observed strain per element were -0.86 and 2.88 for the inside and -0.11 and 0.10 for the outside sensor. However, these numbers are extreme situations for a single element. Furthermore, the inside sensor has higher values compared to the outside sensor in this FEM analysis due to friction between the strap and the phantom finger. The minimum and maximum observed strain of the complete paths of the strain gauge are -0.07 and 0.63 over all situations. As also visualized in Figure 5.4, a considerable contribution of the resistance change is caused by a negative strain (i.e., compression). The presented model assumes this compression will contribute to an increase in the strain gauge resistance as indicated in Figure 5.9. Although the comparison of the model outcomes to the experimental characterization show large similarities, this effect is not proven in a systematic experiment, dedicated to the behavior of the conductive TPU material under compression. Furthermore, in the performed strain test only an axial tensile strain was applied whereas in the sensor structure both compressive and tensile strain are present in multiple directions making the situation even more complex.

Another approach would be to measure the bending of the strap by measuring the difference between the strain on the inside and on the outside of the strap. This approach has been demonstrated by Tognetti et al. [242] using piezoresistive fabric to measure joint angles. These structures are not sensitive to elongation [242] and for example allow the finger sensor to be fitted prestrained around the fingertip. Additionally, differential measurements have been shown to decrease nonlinearities in the sensor response of 3D printed piezoresistive sensors [35]. However, this differential strain gauge approach results in an at least $50 \%$ thicker strap due to the additional strain gauges and insulation, reducing the flexibility and thus reducing the touch sensation of the user.

In the FEM model the extreme inside and outside edges were selected, whereas the actual inside or outside sensor covers the complete area ranging from outer edge to the center line. Therefore, the physical sensors experience a slightly different, less extreme, strain profile compared to the results of the FEM model. Nevertheless, the FEM model, especially in the shear force cases, clearly shows that the curved shape of the sensing structure strain gauges contributes to the relation between sensor output and applied (shear) force.

The applied force in this experimental setup was limited to $10 \mathrm{~N}$ interaction forces while handling objects could reach up to $10 \mathrm{~N}$ for shear forces and up to $50 \mathrm{~N}$ for normal forces [243]. The high sensitivity range in the human touch sensation for both normal and shear forces goes up to $10 \mathrm{~N}$ [14, 237]. Due to the nonlinear effects on the resistance of the TPU material, the accuracy of the sensor is limited. Current research on compensation methods will help to 
improve the accuracy of these strain-sensitive TPU sensors [28]. These methods are, for example, based on analogous electrical models fitted to experimental data. In the future these sensor systems might be paired with smart algorithms or neural networks to exploit the full potential of the measured data. Nevertheless, without the use of these compensation methods and the limited sensitivity for positive normal forces, printed force-sensing structures already have the potential to be used in practical applications. Lifting an object like, e.g., a cup, will induce a shear force at the fingertips in the direction of the gravitational force of the object. This signal can be used for grasp detection in movement sensing systems [19].

Furthermore, the high level of customizability of 3D printing allows for improved sensor geometries that, for example, will minimally cover the skin of the fingertip, allowing for direct skin contact with the object. Both the customizability and the compliance of the soft TPU materials that allow the tactile experience contribute to the high potential of $3 \mathrm{D}$ printed wearable force sensors in a growing market for wearable flexible sensors [244].

\subsection{Conclusions}

This work demonstrates a working concept of a 3D printed flexible sensor capable of measuring shear forces. The sensitivity of the current design for normal forces is limited when used as a finger sensor. The normal and shear force components can be separated. However, creating an inverse model is limited since this relation is non-invertible. A finite element model of the sensing structure and sensing setup shows that the output of the sensor is largely related to the chosen sensor design and strain gauge location. Further deviations probably originate from hysteresis and other nonlinearities of the carbon-infused thermoplastic polyurethane that was used. Characterization of this material shows that the gauge factor of the material depends on the amount of strain and shows strong evidence of being time- and strain-rate-dependent. Within the range of strain estimated by the FEM model, the conductive material shows a negative gauge factor.

Future improvements of the sensor are to be made by using advanced algorithms for signal analysis, or neural networks, to use the full potential of the sensor output. 3D printing offers low-cost and easy customization of sensing structures, which in combination with the soft materials makes these sensors a promising candidate for soft robotics and biomedical applications such as finger interaction sensing. 


\title{
Chapter 6
}

\section{Evaluation of a 3D printed soft sensor for mea- suring fingertip interaction forces}

\begin{abstract}
Current force sensors used to capture fingertip interaction forces lack compliance to the fingertip tissue resulting in the loss of touch sensation of the user. 3D printing offers the possibility to create personalized soft sensing structures. This work evaluates a 3D printed soft sensor that measures normal and shear interactions forces based on the deformations of the thumb and index fingertips of 7 subjects using an instrumented object. Due to the use of (carbon doped) thermoplastic materials, the signals provided by these sensing structures suffer from nonlinearities. Therefore, two compensation models, based on a neural network and recurrent neural network analogous to an electrical model are used to compensate for the nonlinear effects. The performance of the sensors was analysed using the normalized cross-correlation and the root-mean-square error. The output of the force sensors are highly correlated with the applied shear and normal force components. When paired with compensation models the correlation and error of the sensor output can be further improved. These results indicate that the proposed flexible fingertip interaction force sensors have a high potential for future applications.
\end{abstract}

Manuscript submitted for publication: G. Wolterink, M. Schouten, D. Kosmas, B.J. van Beijnum, P. Veltink, G. Krijnen, Evaluation of A 3D printed Soft Sensor for Measuring Fingertip Interaction Forces, 


\subsection{Introduction}

Information about the interaction forces between the human fingertips and the external environment is important for a variety of applications such as sports, haptic devices, robotics and rehabilitation $[236,18]$. In stroke rehabilitation research, robotics and, more recently, kinematic measurements are used to objectively quantify upper limb motor function [9]. Adding information about normal and shear interaction forces at the fingertips during grasping tasks to the kinematic measures, is expected to highly improve the clinical relevance of these measurement systems, since these systems can provide more insight in movement control and effectiveness, allowing for further study on grasp control deficits in stroke subjects [19].

One approach to study the interaction grasping forces is by means of sensorized objects. These devices consist of faces built around the core of a force/torque transducer $[16,17]$ or have a shell of multiple piezoresistive force sensors [245, 246]. Using a matrix of force sensors allows for freedom of sensor placement and the recording of individual finger interaction forces. Although these sensorized objects give a reliable measure of the interaction forces, they are limited by the number of grasping strategies that can be used to manipulate the object and therefore do not represent a wide variety of daily tasks with ordinary objects. The second approach is by mounting force sensors on the fingertips. Such sensors have been developed e.g. by Brookhuis et al [247] and demonstrated by Kortier et al. [18] and Battaglia et al. [14]. Although, these interaction force sensors provide detailed information and can capture a wide range of grasping tasks, these sensors are not ideal to use on the fingertips since they are not compliant to the shape and stiffness of the fingertips resulting in poor sensor to skin attachment and the loss of touch sensation of the user [18] limiting the usefulness and reliability [19].

The compliance of force sensors can be increased in various ways, including the use of flexible circuits, soft and flexible packaging [248], the combination of soft and compliant polymers with flexible conductors such as conductive textiles [249], conductive liquids and inks [250, 94, 237] or by the use of conductive flexible polymers [87, 251]. The emerging developments in 3D printing sensors and the increasing availability of soft, flexible and conductive materials $[81,28,124]$ make this technology a suitable candidate for the development of compliant fingertip sensors [94, 251]. The ability for local manufacturing i.e. distributed manufacturing and the easy to fabricate and customize sensors offered by $3 \mathrm{D}$ printing makes this technology an ultimate candidate to fabricate personalized sensing structures [28]. 


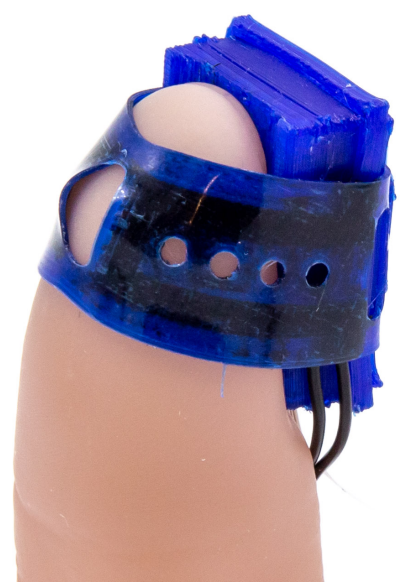

Figure 6.1: $3 \mathrm{D}$ printed finger sensor mounted on a fingertip.

This work presents the evaluation of soft 3D printed fingertip sensors that use mechanical deformation of the soft tissue around the fingertip bone (distal phalanx), during grasping tasks. A preliminary version of this sensor was introduced and investigated using a finite element model and mechanical measurement setup in [251]. That work showed that the output of these sensors is related to the design of the sensing structure and to the position of the strain gauges. Hysteresis and other nonlinearities in the sensor output are associated with the used carbon infused thermoplastic polyurethane materials. Moreover, the gauge factor of these materials is dependent on the amount of strain, the strain rate and time.

This work introduces an adapted sensor design that allows for an improved fit on fingertips and evaluates the sensor for the first time on human fingertips using an instrumented object as reference. Additionally, this works aims to improve the signal quality of the 3D printed sensor with the use of strategies that compensate for the nonlinear effects caused by the material properties and geometry of the fingertip sensor.

\subsection{Sensor design}

Figure 6.1 shows the design of the fingertip sensing structure. This design is based on an earlier concept of the finger sensor published by Wolterink et 


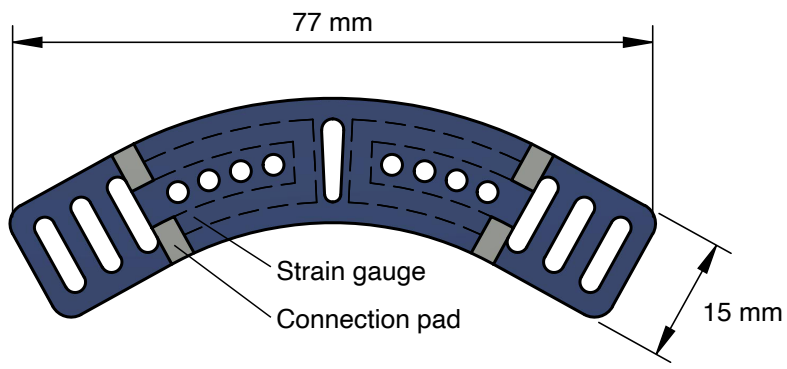

Figure 6.2: Flexible strap of the $3 \mathrm{D}$ printed finger sensor with embedded strain gauges. The three slots at the end can be used to change the lenght of the strap. The strap has a thickness of $0.6 \mathrm{~mm}$ and the strain gauge thickness is $0.20 \mathrm{~mm}$.

al. [251]. The presented sensor concept uses the mechanical deformation of the finger tissue around the bone of the fingertip (distal phalanx). This deformation is sensed by a flexible strap with two embedded strain gauges, wrapped around the fingertip [251]. To improve the fit on human fingertips the sensing strap is designed in an arch shaped geometry (Figure 6.2). The ends of the strap mount to a mounting plate that fits on top of the nail and can be changed in length according to the subjects finger size. The strap has a width of $15 \mathrm{~mm}$ and a thickness of $0.6 \mathrm{~mm}$ and is made from non-conductive TPU (NinjaFlex, Fenner Drives, Manheim, PA, USA). The embedded strain gauges have a thickness of $0.20 \mathrm{~mm}$ and are made of carbon doped conductive TPU (PI-ETPU, Palmiga Innovation, Jonstorp, Sweden). To allow for easy electrical connection the ends of the strain gauges have connection pads that reach out of the strap.

\subsection{Methods}

\subsubsection{Fabrication}

The fingertip sensing structures were printed in one go using a Diabase H-Series Hybrid (Diabase Engineering, USA) multi-material FDM 3D printer. Control and printing settings were handled by the slicer software (Simplify3D, Inc., USA). The layer height of the structure was set to $100 \mu \mathrm{m}$, resulting in a strain gauge with a thickness of two layers. Subsequently, the structures were annealed overnight at $80^{\circ} \mathrm{C}$ in an oven. Electrical interfacing to the strain gauges was made by melting a fine stranded copper wire into the connection pads. The resistance measured between the contact pads of the strain gauges is around $1.8 \mathrm{k} \Omega$. 


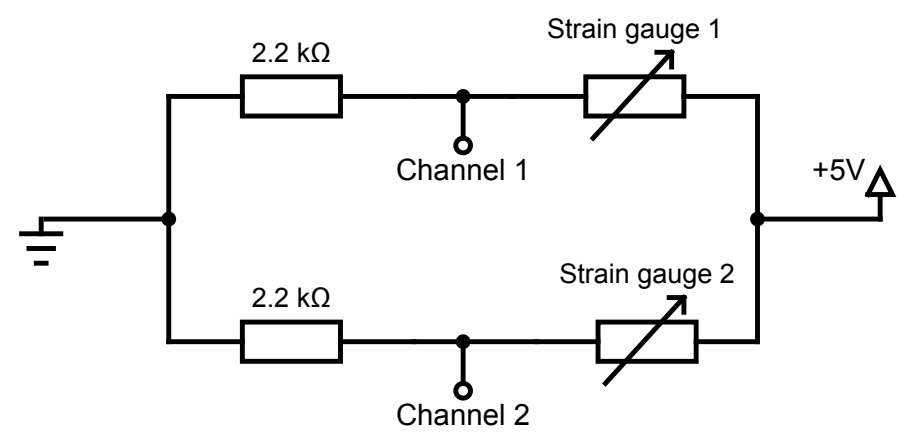

Figure 6.3: Readout circuit of one finger sensor, consisting of two strain gauges. Each strain gauge is read out individually.

\subsubsection{Setup}

To validate the performance of the $3 \mathrm{D}$ printed fingertip sensors during grasping tasks, two identical force sensors were used. One sensor was placed on the thumb and the second sensor was placed on the index finger of the subject. The two strain gauges of each force sensor were read out using voltage divider circuits with $2.2 \mathrm{k} \Omega$ resistors as shown in Figure 6.3. Each channel represents one strain gauge and was read out using the auxiliary inputs of a TMSi Saga amplifier (TMSi, Oldenzaal, The Netherlands) with a sample frequency of $1 \mathrm{kHz}$. The same amplifier was used to provide a voltage of $5 \mathrm{~V}$ to the voltage divider circuit.

The reference force was provided by an instrumented object shown in Figure 6.4. This object was composed of two 6-axis force-torque (FT) sensors (K6D27 50N/1Nm, ME-Meßsysteme GmbH, Hennigsdorf, Germany) mounted at two opposite sides of a stainless steel disk. The FT sensors were read out at a sample frequency of $150 \mathrm{~Hz}$ using two synchronized amplifiers (GSV-8DS, ME-Meßsysteme GmbH, Hennigsdorf, Germany). The metal bases of the force sensors, where the fingertips interact with the object, were covered by a $3 \mathrm{D}$ printed plastic disk with a diameter of $30 \mathrm{~mm}$ for insulation purposes. The distance between the two interaction interfaces was $75 \mathrm{~mm}$. The total weight of the instrumented object was $346 \mathrm{~g}$. A synchronization pulse was provided by a Xsens Awinda Docking Station (Xsens, Enschede, The Netherlands) connected to a personal computer via a USB connection. This pulse was captured by the Saga amplifier and triggers the FT-sensor amplifiers that were all connected to the same computer via a USB connection. 


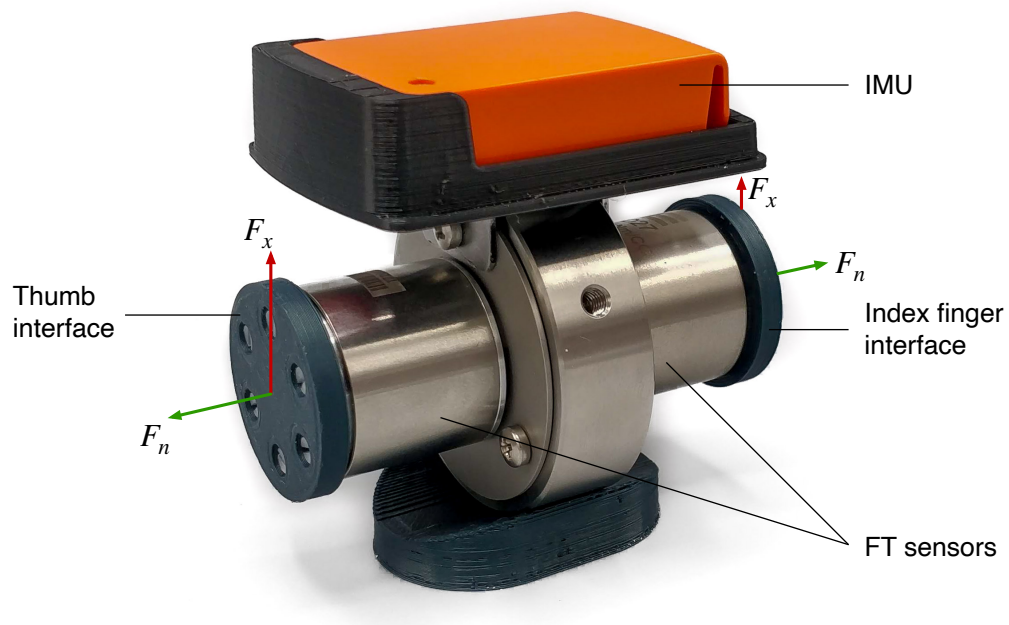

Figure 6.4: The instrumented object used to obtain the reference interaction forces at the fingertips of the thumb and index finger. $F_{\mathrm{n}}$ and $F_{\mathrm{x}}$ represent the normal and shear reaction forces at the fingertips. 


\subsubsection{Protocol}

Seven healthy adult $(18+)$ volunteers participated in the measurements that were approved by the faculty ethics committee, all participants gave written informed consent prior to the start of the experiment. Two 3D printed force sensors were placed on the right hand thumb and index finger of the subjects. The subjects were instructed to perform five recording sessions (tasks 1 to 5) of 3 minutes were the subjects picked up and placed the instrumented object multiple times between the sensorized thumb and index finger at the subject preferred speed. During task 1, 2 and 4 the subjects were asked to pick and place the object multiple times while performing random rotating and shaking movements in random directions, while the instrumented object was being pinched between their thumb and index finger. Task 3 and 5 involved a controlled movement where the subjects were instructed to only pick up and place the object repeatedly for 3 minutes. Between task 3 and 4 the sensors were doffed and re-donned on the fingertips to study the repeatability of the sensors after doffing and donning.

\subsubsection{Data preparation}

All further data processing was performed offline using Matlab (Mathworks Inc., Natick MA, USA). The raw data of the FT sensors were converted to the normal $\left(F_{\mathrm{n}}\right)$ and shear $\left(F_{\mathrm{x}}\right)$ force components using the calibration matrix supplied by the manufacturer. To remove any bias force the mean over the first second, which does not contain any interaction forces, was subtracted of the whole recording. The same procedures were repeated to remove the bias voltage of each channel of the $3 \mathrm{D}$ printed fingertip sensors. Next, both the forces from the instrumented object and the voltage over the strain gauges of the fingertip sensors were low-pass filtered at a cutoff frequency of $10 \mathrm{~Hz}$ using Matlab's lowpass function. Subsequently, the data from the fingertip sensors was re-sampled at $150 \mathrm{~Hz}$ using Matlab's resample function to match the data of the FT sensor. Finally, the recordings of each task were segmented to remove the idle parts before and after each task.

To estimate the force output of the printed sensors, a linear model was fitted using the data set of task 1 for each finger and subject. The fit was performed using Matlab's polyfit function set to a first order polynomial. The normal force sensor output $\left(\tilde{F}_{\mathrm{n}}\right)$ was given by the sum of the two strain gauges. The shear force $\left(\tilde{F}_{\mathrm{x}}\right)$ was given by the difference between the two strain gauges. These models were consequently used to provide an estimate of the normal and shear force of the other performed tasks. 


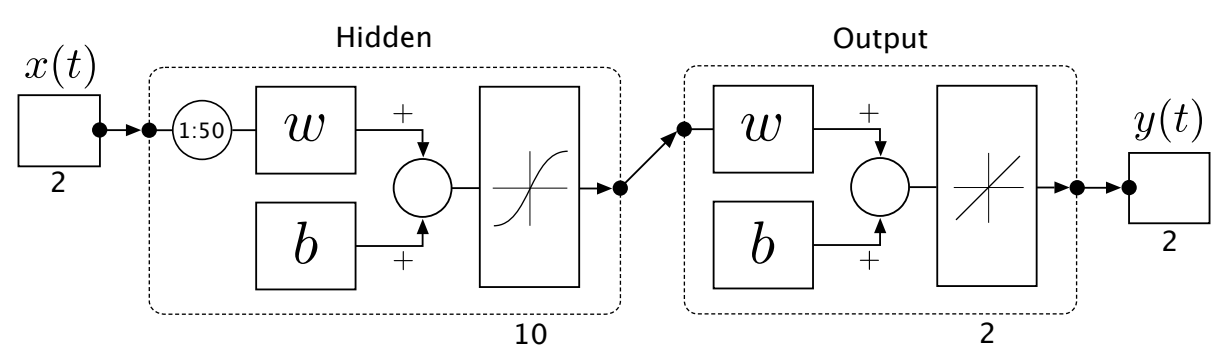

Figure 6.5: Block diagram of the dynamic neural network. The input $(x(t))$ consisted of the 2 inputs from both strain gauges. The output $(y(t))$ are the estimations of both the shear and normal force.

\subsubsection{Nonlinearity compensation}

$3 \mathrm{D}$ printed resistive strain sensors fabricated using soft polymer materials have shown to suffer from strong nonlinear behaviour [28, 251]. To be able to use the finger sensor to its full potential two independent compensation strategies are applied: a neural network approach and a modified Power-Law (mPL) model.

The first compensation strategy involves a dynamic neural network (twolayer feed-forward network), where past values are used to predict future values. These networks can be trained by using a training set with a sufficient number of samples of the two fingertip sensor strain gauges and a target data set. The target dataset contains the applied normal $\left(F_{\mathrm{n}}\right)$ and shear $\left(F_{\mathrm{x}}\right)$ force components of the FT sensor. This network was trained using the Matlab neural network time series toolbox (ntstool) with a nonlinear input-output function [252]

The second compensation model is based on the work of Schouten and Kosmas et al. [196, 253] and is referred to as the modified Power-Law (mPL) model. The mPL model estimates the hysteresis and creep behaviours using a recurrent neural network that is analogous to a the forward Euler simulation of an electrical circuit. This network also consists of two layers, see Figure 6.6. The first layer uses a hyperbolic sine $f(x)=P_{\mathrm{S}}\left(e^{\frac{x}{P_{\mathrm{T}}}}-e^{\frac{-x}{P_{\mathrm{T}}}}\right)$ as activation function. The output layer uses a 4 th order polynomial $g(x)=g_{0}+g_{1} x \ldots g_{m} x^{m}$. Both weights and the parameters of both activation functions are included in the optimization. The used fitting method is based on Matlab's optimization algorithm for obtaining a function's global minimum [254]. The global problem is defined as multiple local optimization problems by means of GlobalSearch, with the algorithm's default configuration [255] and the fmincon method configured with an interior-point algorithm [256] as described by Kosmas et al. [253]. 
Hidden

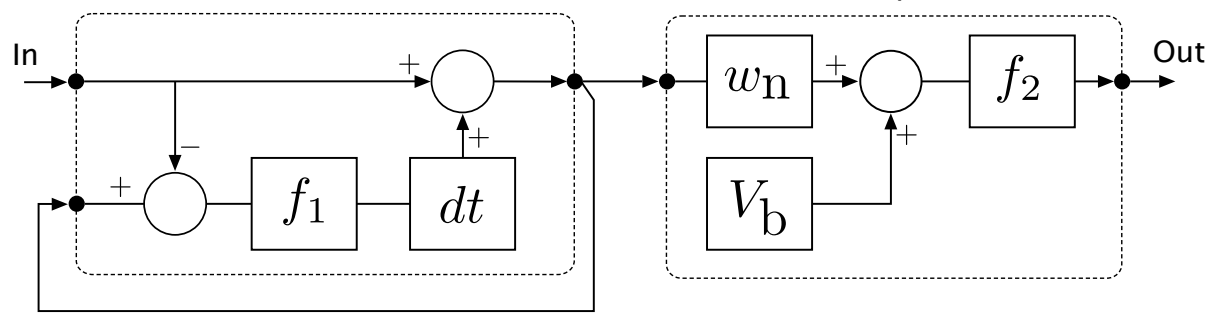

Figure 6.6: Block diagram of the mPL model.

\section{Model training and validation}

Two types of training and validation were performed, to investigate if a generic inter subject model for the sensor is sufficient or whether each subject needs an individual model (intra subject model). Inter subject models are based on a model trained on the task 1 data set of subject 1 and were validated using all tasks of all subjects. Intra subject models involve models trained on task 1 of each individual subject and are only validated by the tasks performed by the same individual subject. For both the thumb and the index finger sensor a separate compensation model was trained to avoid for potential differences in sensor size or printing inaccuracies. In all situations the forces obtained form the FT sensors were used as target data.

The Matlab neural network time series toolbox (ntstool) with a nonlinear input-output was used to train the neural networks. The input data consisted of 2 input signals, each representing one strain gauge of the sensor. $F_{\mathrm{n}}$ and $F_{\mathrm{x}}$ of the FT sensor represent the two elements of the target data. The network consists of 10 hidden neurons and 50 delays. This number is found as a good balance between training time and performance.

Two separate modified Power-Law ( $\mathrm{mPL}$ ) compensation models were trained, the first model estimates the normal force $F_{\mathrm{n}}$ using the sum of the two strain gauges as input. The second model estimates the shear force $F_{\mathrm{x}}$ using the differential signal between the two strain gauges. The weights $w_{0}, w_{1}$, bias $V_{\mathrm{b}}$, weights $P_{\mathrm{T}}$ and $P_{\mathrm{S}}$ of the hyperbolic sine function [253], along with the coefficients $g_{0} \ldots g_{m}$, were estimated using the experimental data for training and validation. The initial parameters before fitting are shown in Table 6.1. To speed up the training the data was down-sampled by a factor 2 , which resulted in a time step $\Delta t$ of $13.3 \mathrm{~ms}$. 
Table 6.1: Initial parameters of the mPL model.

\begin{tabular}{l|l} 
Parameter & Value \\
\hline$w_{0}$ & 1 \\
$w_{1}$ & -0.1 \\
$V_{\mathrm{b}}$ & $0.1 \mathrm{~V}$ \\
$P_{\mathrm{T}}$ & 0.2 \\
$P_{\mathrm{S}}$ & 0.2 \\
$g_{0} \ldots g_{5}$ & 0.1
\end{tabular}

\subsubsection{Statistiscs}

The correlation between the output of the models and the validation data was determined by the normalized cross-correlation (NCC). Since the model input and validation data are synchronized, the NCC values were taken at zero lag for further analysis. The error between the model output and validation data was calculated using the root-mean-square error (RMSE). The Wilcoxon signed rank test was used to determine whether the NCC and RMSE values between tasks, validated using the compensation models differ or not. This measure was furthermore used to validate differences in performance between the applied compensation models. The Wilcoxon signed rank test was chosen due to the expected non-normal distributed data and small sample size.

\subsection{Results}

All seven participants completed the experimental protocol. The performance data of the second random movement (task 2) of subject 5 was discarded due to a malfunction in the synchronization between the finger sensors and the instrumented object. The finger sensor straps of the thumb were to their maximum size (3) for subjects $1,2,3,6$ and to medium (2) for subjects $4,5,7$, the straps for the index finger were set to medium length (2) for all subjects.

Figure 6.7 shows parts of the training and validation data recorded at the thumb, the graphs on the left represent normal forces and the graphs at the right represent the shear forces. The low pass filtered output of the strain gauges of task 3 performed by subject 7 is shown in Figure $6.7 \mathrm{a}, \mathrm{b}$. The same fragment is shown again in the bottom graphs $(e, f)$ after the compensation models trained on task 1 of subject $1(\mathrm{c}, \mathrm{d})$, are applied.

Figure 6.8 shows the output of a linear fit performed on task 1 of each subject for the normal force $\left(F_{\mathrm{z}}\right)$. This fit is determined by the sum of both strain gauges $(\mathrm{Ch} 1+\mathrm{Ch} 2)$. In the shear force $\left(F_{\mathrm{x}}\right)$ situation this fit is made 

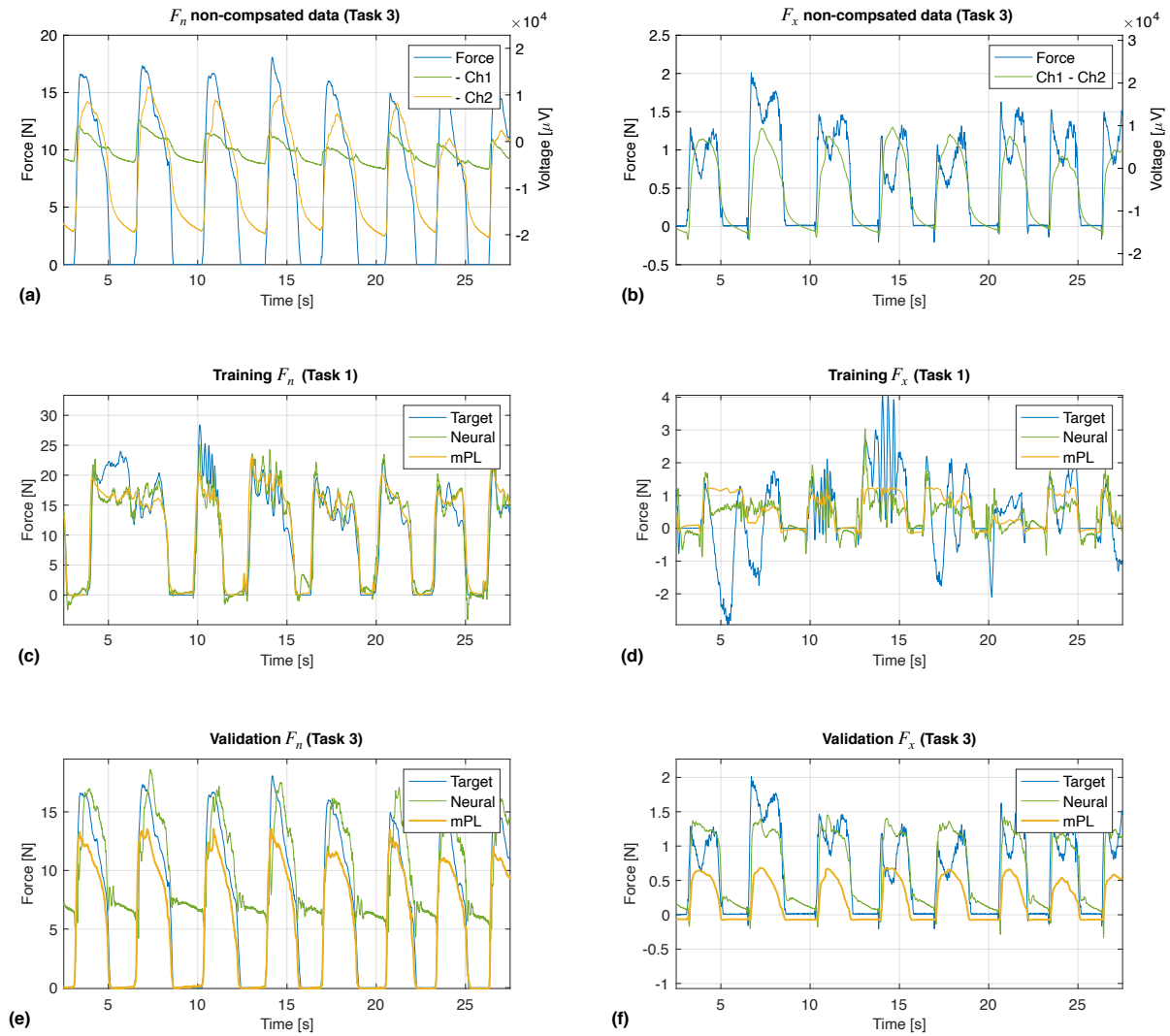

Figure 6.7: Time domain data recorded from the instrumented object and fingertip sensors at the thumb. Left figures represent normal forces, right figures shear forces. The compensation models are trained on task 1 of subject $1(\mathrm{c}, \mathrm{d})$. The noncompensated data $(\mathrm{a}, \mathrm{b})$ and validation $(\mathrm{e}, \mathrm{f})$ are taken from subject 7 , task 3 . 

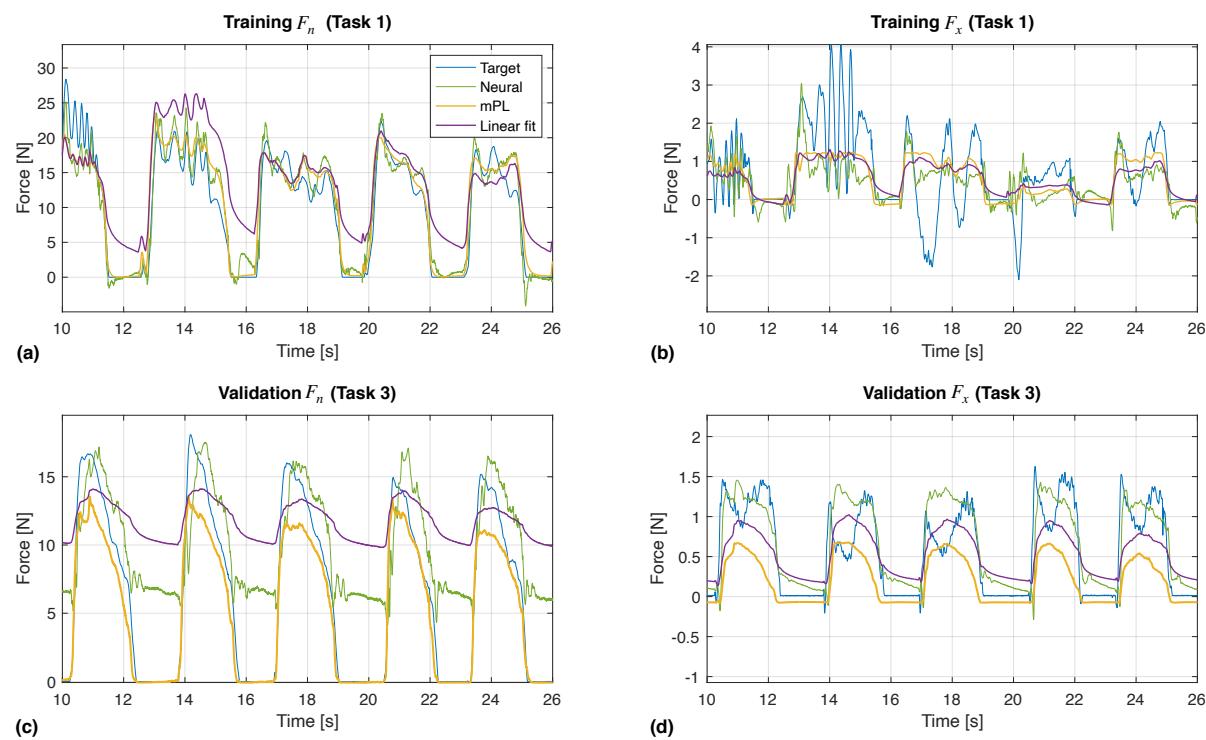

Figure 6.8: Time domain data with a close up of the same fragments as shown in Figure 6.7 including the output provided by a linear fit performed on task 1 .

using the difference between the two strain gauges (Ch1 - Ch2).

Since no significant difference $(P<0.05)$ in normalized cross-correlation (NCC) at zero lag at the training sets of the thumb and index finger for both the neural net and the modified Power-Law model (mPL) were found, the performance of the thumb and index finger were grouped in further analysis to double the number of measurements. Each finger sensor (thumb and index) is still compensated with the compensation model for that sensor.

Figure 6.9 shows the box plots of the NCC at zero lag for both normal force $\left(F_{\mathrm{z}}\right)$ and the shear force $\left(F_{\mathrm{x}}\right)$ data obtained from each task of the non compensated data (Linear fit) and analysed using a neural network (Neural Inter/Neural Intra) or the modfied Power-Law model (mPL Inter/mPL Intra). The suffix 'inter' refers to models trained on subject 1 task 1 and applied on all subjects, suffix 'intra' refers to models trained on each individual subject on task 1 .

The significance of the differences between the data presented in Figure 6.9 is shown in Figures 6.10 to 6.12. Figure 6.10 shows the significance of the differences of the NCC between the linear fit and the compensation models. Figure 6.11 shows the significance of the differences between compensation models using the same analysis method and Figure 6.12 shows this significance of the 
differences between the performed tasks.

Figure 6.13 shows the root-mean-square error (RMSE) for both normal force $\left(F_{\mathrm{z}}\right)$ and the shear force $\left(F_{\mathrm{x}}\right)$. The graph clearly shows an order of magnitude lower RMSE for the sensor output compensated with the mPL models with respect to the linear fit and the output compensated using a neural network. The corresponding $P$-values of the differences between the linear fit and the models are shown in Figure 6.14. Figure 6.15 shows the statistical power between the compensation models and Figure 6.16 between the tasks. 

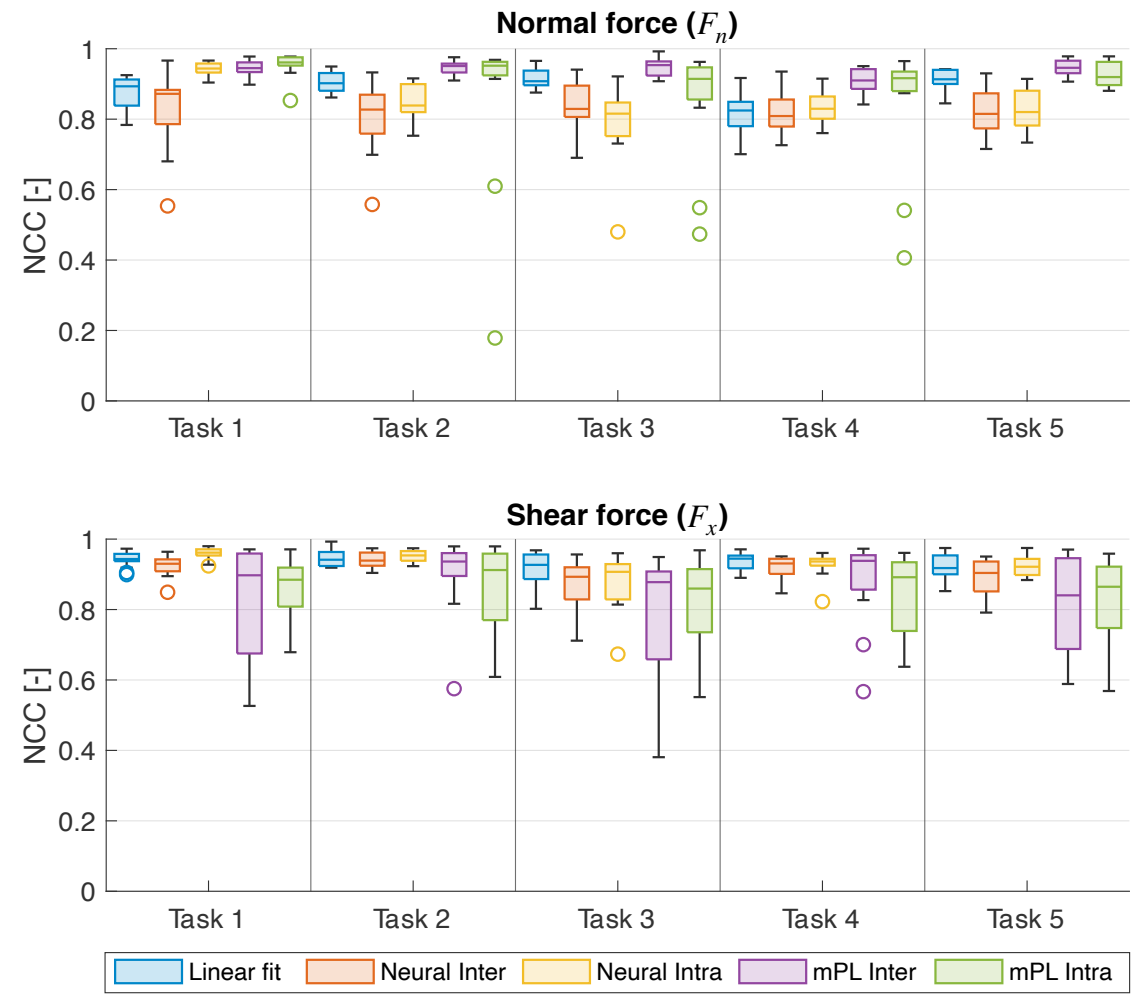

Figure 6.9: Box plots of the normalized cross-correlation (NCC) at zero lag for both normal and shear force data. Results from the linear fit on the non compensated data of task 1 (Linear fit) and both models, the neural network (Neural) and modified Power-Law (mPL) model are shown. 'Inter' refers to one general model trained on task 1 of subject 1 and applied on all tasks of all subjects. 'Intra' refers to a network trained for each individual subject on task 1 . Outliers are represented by the open dots. 


\begin{tabular}{r|c|c|c|c|c|}
\multicolumn{4}{c}{} & \multicolumn{5}{c}{ P-value between models and Linear fit (NCC) } \\
\cline { 2 - 6 } Neural Inter & 0.1 & 0.01 & 0.01 & 1 & 0.01 \\
\cline { 2 - 6 } Neural Intra & 0.01 & 0.01 & 0.01 & 0.63 & 0.01 \\
\cline { 2 - 6 } mPL Inter & 0.01 & 0.01 & 0.01 & 0.01 & 0.01 \\
\cline { 2 - 6 } mPL Intra & 0.01 & 0.23 & 0.46 & 0.12 & 0.1 \\
\cline { 2 - 6 } & & & & & \\
\cline { 2 - 6 } & 0.1 & 0.57 & 0.01 & 0.46 & 0.01 \\
\cline { 2 - 6 } Neural Inter & 0.57 & 0.19 & 0.86 & 1 \\
\cline { 2 - 6 } Neural Intra & 0.05 & 0.52 & 0.05 & 0.3 & 0.05 \\
\cline { 2 - 6 } mPL Inter & 0.05 & 0.05 & 0.05 & 0.05 & 0.01 \\
\cline { 2 - 6 } mPL Intra & 0.05 & 2 & 3 & 4 & 5 \\
\cline { 2 - 6 } & 1 & & Tasks & &
\end{tabular}

Figure 6.10: $P$-value between the linear fit and the compensation models as shown in Figure 6.9.

\begin{tabular}{|c|c|c|c|c|c|}
\hline \multirow{3}{*}{$\begin{array}{l}\text { Neural Inter - Neural Intra } \\
\text { mPL Inter - mPL Intra }\end{array}$} & \multicolumn{5}{|c|}{ P-value between models (NCC) } \\
\hline & 0.01 & 0.13 & 0.15 & 0.52 & 0.52 \\
\hline & 0.05 & 0.92 & 0.01 & 0.68 & 0.01 \\
\hline \multirow{2}{*}{$\begin{array}{l}\text { Neural Inter - mPL Inter } \\
\text { Neural Intra - mPL Intra }\end{array}$} & 0.01 & 0.01 & 0.01 & 0.01 & 0.01 \\
\hline & 0.1 & 0.2 & 0.17 & 0.12 & 0.01 \\
\hline \multirow{5}{*}{$\begin{array}{r}\text { Neural Inter - Neural Intra } \\
\text { mPL Inter - mPL Intra } \\
\text { Neural Inter - mPL Inter } \\
\text { Neural Intra - mPL Intra }\end{array}$} & 0.01 & 0.23 & 0.79 & 0.34 & 0.1 \\
\hline & 0.42 & 0.38 & 0.2 & 0.3 & 0.91 \\
\hline & 0.15 & 0.85 & 0.33 & 0.67 & 0.14 \\
\hline & 0.01 & 0.1 & 0.39 & 0.1 & 0.05 \\
\hline & 1 & 2 & $\begin{array}{c}3 \\
\text { Tasks }\end{array}$ & 4 & 5 \\
\hline
\end{tabular}

Figure 6.11: $P$-value showing the significant differences in NCC values at zero lag as shown in Figure 6.9 between compensation models at each task.

\begin{tabular}{|c|c|c|c|c|c|c|}
\hline \multirow[b]{2}{*}{ Linear fit } & \multicolumn{6}{|c|}{ P-value between tasks (NCC) } \\
\hline & 0.01 & 0.05 & 0.01 & 0.05 & 0.01 & 0.9 \\
\hline \multirow{4}{*}{$\begin{array}{c}\text { Neural Inter } \\
\text { Neural Intra } \\
\text { mPL Inter } \\
\text { mPL Intra }\end{array}$} & 0.27 & 0.81 & 0.46 & 0.9 & 0.73 & 0.24 \\
\hline & 0.01 & 0.01 & 0.01 & 0.01 & 0.2 & 0.24 \\
\hline & 0.52 & 0.71 & 0.01 & 0.67 & 0.05 & 1 \\
\hline & 0.05 & 0.01 & 0.01 & 0.05 & 0.13 & 0.05 \\
\hline \multirow{6}{*}{$\begin{array}{r}\text { Linear fit } \\
\text { Neural Inter } \\
\text { Neural Intra } \\
\text { mPL Inter } \\
\text { mPL Intra }\end{array}$} & 0.42 & 0.1 & 0.43 & 0.1 & 0.05 & 0.71 \\
\hline & 0.1 & 0.05 & 0.86 & 0.05 & 0.1 & 0.43 \\
\hline & 0.27 & 0.01 & 0.01 & 0.01 & 0.05 & 0.1 \\
\hline & 0.23 & 0.39 & 0.54 & 0.81 & 0.68 & 0.19 \\
\hline & 0.97 & 0.19 & 0.5 & 0.1 & 0.3 & 0.54 \\
\hline & $1-2$ & $1-3$ & $1-4$ & $1-5$ & $2-4$ & $3-5$ \\
\hline
\end{tabular}

Figure 6.12: $P$-value showing the significant differences between the NCC value at zero lag of the performed tasks in Figure 6.9. Task 1 is the training data in all situations. 

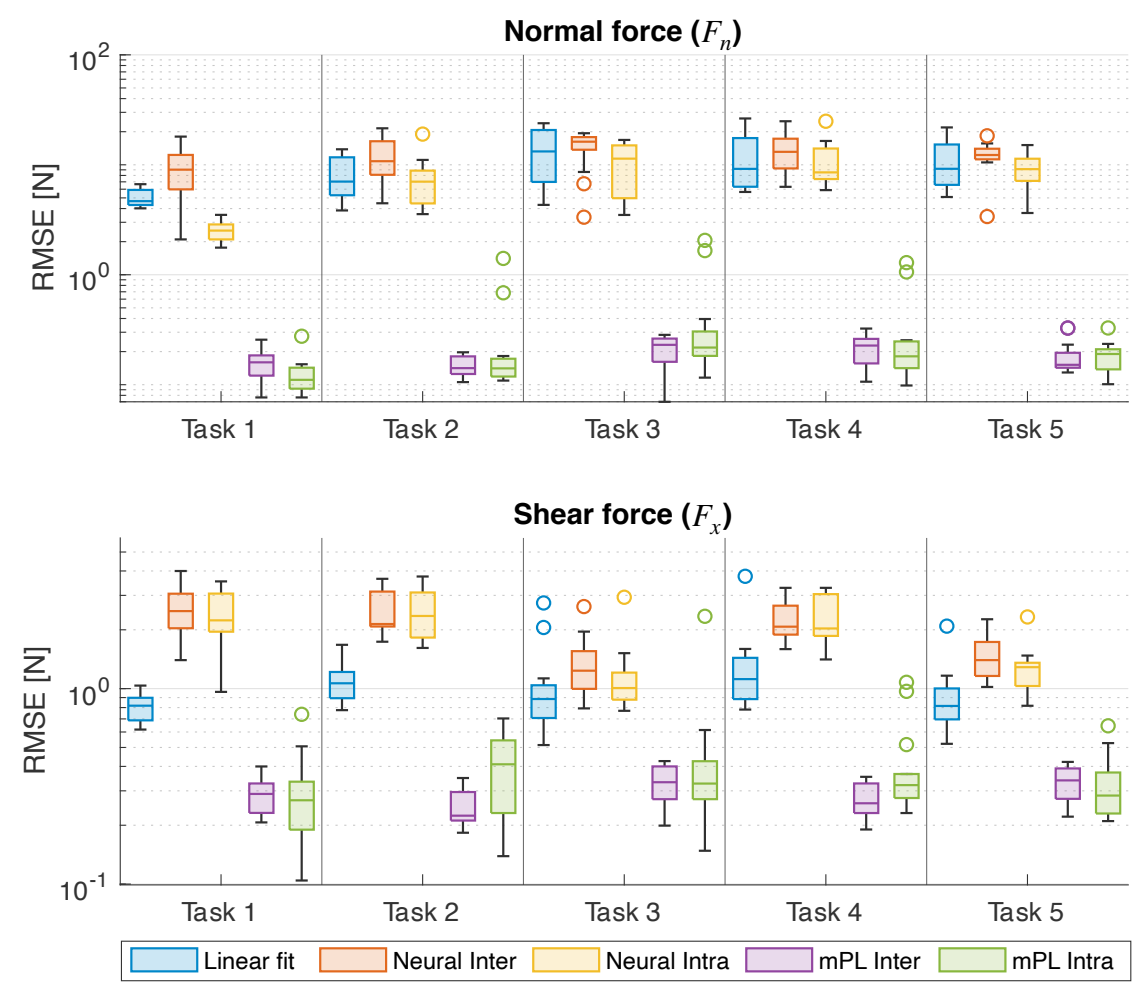

Figure 6.13: Box plots of the root-mean-square error (RMSE) for both normal and shear force data. Results from the linear fit on the non compensated data of task 1 (Linear fit) and both models, the neural network (Neural) and modified Power-Law (mPL) model are shown. 'Inter' refers to one general model trained on task 1 of subject 1 and applied on all tasks of all subject. 'Intra' refers to a network trained for each individual subject on task 1 . Outliers are represented by the open dots. 


\begin{tabular}{r|c|c|c|c|c|}
\multicolumn{2}{c}{} & \multicolumn{5}{c}{ P-value between models and Linear fit (RMSE) } \\
\cline { 2 - 6 } Neural Inter & 0.01 & 0.11 & 0.67 & 0.22 & 0.19 \\
\cline { 2 - 6 } Neural Intra & 0.01 & 0.1 & 0.01 & 0.9 & 0.19 \\
\cline { 2 - 6 } mPL Inter & 0.01 & 0.01 & 0.01 & 0.01 & 0.01 \\
\cline { 2 - 6 } mPL Intra & 0.01 & 0.01 & 0.01 & 0.01 & 0.01 \\
\cline { 2 - 6 } & & & & & \\
\cline { 2 - 6 } & 0.01 & 0.01 & 0.1 & 0.01 & 0.01 \\
\cline { 2 - 6 } Neural Inter & 0.01 & 0.01 & 0.17 & 0.05 & 0.05 \\
Neural Intra & $F_{x}$ \\
mPL Inter & 0.01 & 0.01 & 0.01 & 0.01 & 0.01 \\
\cline { 2 - 6 } mPL Intra & 0.01 & 0.01 & 0.01 & 0.01 & 0.01 \\
\cline { 2 - 6 } & 1 & 2 & 3 & 4 & 5 \\
\cline { 2 - 6 } & & & Tasks & &
\end{tabular}

Figure 6.14: $P$-value between the linear fit and the compensation models as shown in Figure 6.13.

P-value between models (RMSE)

\begin{tabular}{r|c|c|c|c|c|}
\cline { 2 - 6 } Neural Inter - Neural Intra & 0.01 & 0.05 & 0.01 & 0.27 & 0.01 \\
\cline { 2 - 6 } mPL Inter - mPL Intra & 0.05 & 0.63 & 0.27 & 0.62 & 0.52 \\
\cline { 2 - 6 } Neural Inter - mPL Inter & 0.01 & 0.01 & 0.01 & 0.01 & 0.01 \\
\cline { 2 - 6 } Neural Intra - mPL Intra & 0.01 & 0.01 & 0.01 & 0.01 & 0.01 \\
\cline { 2 - 6 } & & & & & \\
\cline { 2 - 6 } & 0.05 & 0.43 & 0.1 & 0.85 & 0.1 \\
\cline { 2 - 6 } Neural Inter - Neural Intra \\
mPL Inter - mPL Intra & 0.79 & 0.01 & 0.47 & 0.01 & 0.47 \\
\cline { 2 - 6 } Neural Inter - mPL Inter & 0.01 & 0.01 & 0.01 & 0.01 & 0.01 \\
\cline { 2 - 6 } \\
Neural Intra - mPL Intra & 0.01 & 0.01 & 0.01 & 0.01 & 0.01 \\
\cline { 2 - 6 } & 1 & 2 & 3 & 5 \\
\end{tabular}

Figure 6.15: $P$-value showing the significant differences in RMSE as shown in Figure 6.13 between compensation models at each task.

\begin{tabular}{|c|c|c|c|c|c|c|}
\hline \multirow[b]{2}{*}{ Linear fit } & \multicolumn{6}{|c|}{ P-value between tasks (RMSE) } \\
\hline & 0.01 & 0.01 & 0.01 & 0.01 & 0.2 & 0.1 \\
\hline \multirow{4}{*}{$\begin{array}{c}\text { Neural Inter } \\
\text { Neural Intra } \\
\text { mPL Inter } \\
\text { mPL Intra }\end{array}$} & 0.15 & 0.05 & 0.1 & 0.05 & 0.42 & 0.17 \\
\hline & 0.01 & 0.01 & 0.01 & 0.01 & 0.05 & 0.46 \\
\hline & 0.85 & 0.1 & 0.05 & 0.43 & 0.05 & 0.19 \\
\hline & 0.01 & 0.01 & 0.01 & 0.01 & 0.23 & 0.01 \\
\hline \multirow{6}{*}{$\begin{array}{r}\text { Linear fit } \\
\text { Neural Inter } \\
\text { Neural Intra } \\
\text { mPL Inter } \\
\text { mPL Intra }\end{array}$} & 0.01 & 0.67 & 0.01 & 0.81 & 0.52 & 0.67 \\
\hline & 0.47 & 0.01 & 0.58 & 0.01 & 0.1 & 0.14 \\
\hline & 0.2 & 0.01 & 0.81 & 0.01 & 0.27 & 0.14 \\
\hline & 0.13 & 0.05 & 0.71 & 0.01 & 0.42 & 0.95 \\
\hline & 0.1 & 0.05 & 0.05 & 0.1 & 0.79 & 0.24 \\
\hline & $1-2$ & $1-3$ & $1-4$ & $1-5$ & $2-4$ & $3-5$ \\
\hline
\end{tabular}

Figure 6.16: $P$-value showing the significant differences between the RMSE of the performed tasks in Figure 6.13. Task 1 is the training data in all situations. 


\subsection{Discussion}

\subsubsection{Nonlinearity compensation}

The time domain data shown in Figures 6.7 and 6.8 indicate a high correlation between the target data provided by the instrumented object and the sensor output. As shown by Figure 6.9 the use of compensation models further increases the correlation of the printed fingertip sensors with the reference force, this effect was mostly significant for the mPL models as shown in Figure 6.10.

Although, the linear fit shows a high correlation with the reference signal, Figure 6.8 shows a large offset between the reference force and output of the linear fit. This offset is mainly caused by three factors: lower amplitude, drift and relaxation time. The last effect is strongly visible at the situation where the applied force rapidly decreases and is a typical behaviour for the used conductive polymer composite materials $[240,124,251]$. The relaxation time and drift are largely eliminated with the use of the neural networks and $\mathrm{mPL}$ models. As shown in Figure 6.13 the used mPL models provide a significant $(p \leq 0.01)$ lower root-mean-square error (RMSE) and therefore these models seem to be the best compensation strategy for this sensor and experiment.

Notwithstanding the fact that the performed analysis allows to make a comparison between the performance of neural networks with respect to the $\mathrm{mPL}$ model, no conclusions should be drawn regarding the performance of these type of models. Both compensation models used in this work are preliminary versions using a limited number of parameters and provide many opportunities for improvements.

The mPL model with a single cell is only able to capture hysteresis behaviour described with local memory, since the model's global memory arises as a collective property from the multiple capacitor states when the number of cells is expanded $\left(n_{\text {cell }}>1\right)$ [257]. Whether the hysteresis exhibited by the sensors can be described with local or global memory is yet to be evaluated. To evaluate this in a more systematic way, a generated system input on the finger sensor using a mechanical setup, such as performed on a previous design shown in [251] is needed.

In case of the neural network, care needs to be taken with increasing the number of neurons since the models fitted on normal force data seem to suffer from overfitting. In terms of training speed the neural network needed a computation time of around 4 to 6 minutes to reach satisfying training results. The $\mathrm{mPL}$ model used in this work reached this point usually around one minute. These model fitting times are obtained using a third generation (2.6 GHz Dual- 
Core) Intel Core i5, and highly vary depending on the used amount of training data, parameters, fitting iterations and hardware performance.

\subsubsection{Normal force performance}

As shown in Figure 6.9 and 6.11 the training data (task 1) in the normal force direction, is higher correlated when the models are trained for each individual subject (intra subject). The same effect can be seen in Figure 6.13 and 6.15, where intra subject models show a lower RMSE. This suggests that the compensation models are optimized to individual users. However, in the validation tasks (task 2 to 5) this effect becomes less prominent and significant.

The inter mPL model shows a significantly higher median NCC value on all tasks compared to the neural network for the normal force data. Furthermore, the RMSE of the mPL model is an order of magnitude lower. This all shows that for estimation using a generic sensor model, the mPL model is in this situation, the best option to obtain the normal force component of the 3D printed finger sensors.

\subsubsection{Shear force performance}

In the shear force direction the mPL models show a larger spreading in the NCC values compared to the neural network. This might be due to the higher number of parameters and the ability to train the neural networks using the two separate inputs provided by the strain gauges of the finger sensor, whereas the input for the $\mathrm{mPL}$ model is only the difference between the two strain gauges. However, differential measurements were taken since these can highly increase the linearity of the response for $3 \mathrm{D}$ printed piezoresistive sensors [35]. The RMSE in Figure 6.15 shows an opposite trend; the median RMSE of the mPL results is an order of magnitude lower compared to the linear fit and neural networks and in the same range as the RMSE of the normal force. However, most of the signal power is in the normal component, as shown in Figure 6.7 where the amplitude of the normal force components is around one order of magnitude higher than the shear force components. Therefore, the relative errors in the shear force situation are an order of magnitude higher than in the normal force situation. 


\subsubsection{Influence of re-donning}

In the situation where re-donning the $3 \mathrm{D}$ printed sensors affects the performance of the sensors one might expect a lower NCC and a larger RMSE in the tasks performed after re-donning the sensors. If only one compensation model is used for multiple subjects, the sensors are re-donned between subsequent subjects and could therefore be a factor reducing the performance. However, the results do not provide an evident conclusion on the effect of re-donning the force sensors.

Figures 6.11 and 6.15 do not show a consequent strong statistical power $(p \leq 0.05)$ of differences between the inter and intra subject fitted compensation models. Figures 6.12 and Figure 6.16 do show small significant differences in some situations between the tasks before and after re-donning (task 2-4 and 3-5), indicating possible performance change caused by re-donning of the sensors. However, the controlled movements before and after re-donning the sensors (task 3 and 5) show a significant decrease of the RMSE after re-donning for the linear fit and $\mathrm{mPL}$ model trained intra subject. For these reasons, the effect of re-donning is not evident and in case a general inter subject fitted compensation model is used, no notable negative effects of re-donning are expected.

\subsubsection{Limitations}

Besides the used thermoplastic material in the finger sensor, the human fingertip tissue is also a highly nonlinear material with characteristics comparable to viscoelastic materials [258, 259]. Since the skin tissue is part of the sensing structure these characteristics will be present in the raw sensors output, but potentially are compensated by the presented compensation models.

Despite the possibility offered by 3D printing to develop custom sized sensors fitted to each individual subject's fingertip circumference, this preliminary study uses a strap-like structure to change the size of the fingertip sensor. Since this strap only has three discrete length configurations, the initial strain of this sensor around the subjects fingertip might vary. However, analysis between the inter and intra trained compensation models shows no clear evidence this effect negatively affects the NCC and/or RMSE.

The used instrumented object only contained two 6-axis force-torque sensors, allowing the current experimental setup to only validate pinch grasps, whereas in all day grasping activities the complexity of grasps usually involves more fingertips. Furthermore, in many all day human grasping activities more areas than only the fingertips, such as the phalanges or the hand palm, are also involved by supporting the object. 
The contact area of the force sensors on the instrumented object was relatively small. Although the subjects were instructed to carefully place their complete fingertip on the force sensor area, in some situations it could not be avoided that the subjects only placed a part of their fingertip on the object. Resulting in discrepancies between the force sensors of the instrumented object and the finger sensors.

In some occasions, accidental fingertip interactions with other objects such as the table or the subjects own fingers occurred. These interaction recordings occur in the data sets of the printed sensors and are absent in the validation data provided by the instrumented object, reducing the NCC and increasing the RMSE values since there is no correlating validation signal.

It is assumed that the shear component of the 3D printed finger sensor is perpendicular to the $F_{\mathrm{x}}$-component of the instrumented object. However, the angle between these two components might vary with each grasp. In future research these errors could be compensated by using small inertial sensors placed on the fingertip nails such as described in $[9,19]$, to estimate the orientation of the shear component with respect tot the instrumented object or gravity.

Since the subjects are asked to lift an object, the performed tasks have a large coupling between shear and normal force component due to the inherent functioning of the 3D printed sensor. This coupling could lead to an unwanted trained correlation in the neural networks. To avoid this issue the subjects are asked to shake and rotate the object during the training tasks to generate decoupled forces. However, applying forces to a fixed reference sensor could help to increase the amount of decoupled data.

The soft fingertip sensors are capable of detecting the shear force component; Figure 6.8 clearly shows the increase in shear force component when the instrumented object is lifted. These signals are of high value for the development of grasp detection algorithms that are of relevance for assessment of upper limb impairments [19]. One of the potential applications of these sensing structures is the study of grasp control deficits in stroke patients. In these situations measuring the relative change in grasping force will already be a valuable addition. Therefore, the sometimes relativity large absolute error of the force as measured by the fingertip force sensors, is not necessarily a limitation.

This study has demonstrated a working concept of a soft 3D printed piezoresistive fingertip force sensor. Although the signal output of these sensors is far from perfect relative to commercially available solutions, the compliance of the sensors to the fingertip makes these sensors useful for clinical and all day use. Furthermore, 3D printing of force sensing structures offers benefits over commercial sensors since the fabrication technology allows for easy customization. 
This study does not investigate the users touch sensation, although it is expected that the use of compliant materials to the finger tissue and the open structure at the fingertips allows for improved touch sensation compared to currently available stiff sensors. These benefits highly increase the potential for adoption of this sensing technology.

\subsection{Conclusion}

This work demonstrates the potential of 3D printed flexible fingertip force sensors to be used in practical applications. The correlation and the error between the output of the force sensors and the applied shear and normal force components can be highly improved with the use of models that compensate the nonlinear characteristics of the used sensors.

The signal quality of the fingertip sensors might be improved in the future since the used compensations models are relatively simple and are still in an early development phase. Nevertheless, the current state of these sensors might already be sufficient for elementary grasp detection applications that use the relative change in force. $3 \mathrm{D}$ printed fingertip sensors provide many benefits over commercial sensors due to their compliance and the ability for the users to keep their touch sensation. 


\section{Chapter 7}

\section{Discussion}

The research of this thesis is driven by the main goal to develop and evaluate a distributed sensing system for the upper limbs that integrates EMG, kinematic and kinetic metrics, with the use of new soft flexible personalized sensing structures. The development of soft 3D printed EMG electrodes has been a vehicle for the development of 3D printing with embedded electrically conductive structures for sensing applications. This research has resulted in many insights into the capabilities and limitations of 3D printed sensors. The first part of this chapter discusses the assessment of the upper limbs of stroke subjects using a distributed sensing system. Although this system is equipped with commercial sensors it allows for the integration of the customized soft EMG electrodes and force sensors developed in this work. The development of these sensing structures is initiated by the limitation of currently available force sensors and EMG electrodes. The rapid advancement in 3D printed sensors allows for the development of new soft sensing structures. The challenges and new insights into the development of 3D printed sensors will be discussed in the second section of this chapter. 


\subsection{Motor function assessment using distributed sen- sing systems}

Currently, assessment and evaluation of the recovery of upper limb motor function is performed by therapists through observation and performing clinical tests that are mainly evaluated subjectively. Although, assessment protocols, such as the FMA are used, scores given by a human observer can be influenced by subjective observations and interpretations. Furthermore, these protocols lack fine grain details and suffer from ceiling effects [6]. The use of quantitative measurement tools enables longer recording and objective assessment of upper limb motor function, allowing for improved rehabilitation therapy. Quantitative measurements of motor function can be captured at multiple motor control levels: electromyography (EMG) provides information regarding the neural control of muscle activation, kinematics describe the movement and orientation of the body and kinetic measures involve the interaction forces of the body with the environment.

Studies have shown that several kinematic features have a strong relation with clinical scales $[75,76,48,72,62,260]$. The distributed IMUs of the iHams system presented in Chapter 2 are capable of distinguishing movement between the affected and non-affected side. The relation with FMA-UE arm score is limited and strongly varies among the subjects. This may indicate that the fine-grain information of the IMU sensing system provides complementary information to the FMA-UE clinical scores. However, the meaning and validity of this complementary information of motor function needs to be further investigated with respect to clinical relevance. The movement analysis features described in Chapter 2 are limited to only joint angles. These metrics were chosen in close collaboration with clinicians due to the ease of interpretation and therefore allowing for easy acceptance in the clinic. Future studies could provide a set of essential features that provide the most relevant information regarding upper limb motor function. This set might be obtained by dimensionality reduction techniques such as principal component analysis to limit the amount of redundant variables [261]. Identifying a set of essential features, based on further clinical studies, might help to reduce the obtrusiveness of the system, since a fixed set of clinical relevant features allows for a reduced number of optimallypositioned sensors and, therefore, may reduce the donning and calibration time of the system. In a study of Schwarz et al. [19], the iHams system was shown to be able to detect trunk compensation strategies during object displacement tasks when the stroke subjects use their affected upper limb. The detection of 
compensation strategies could help to further optimize rehabilitation therapy by providing feedback to the patient and therapist.

Electromyography (EMG) has shown to improve the performance of quantitative assessment of rehabilitation therapy when combined with inertial measures [62]. EMG can provide signals correlated with grasping activity and force [260] and with the use of pattern recognition algorithms arm and hand gestures can be identified [235, 90]. Furthermore, EMG can be used to quantify pathological activation patterns during motor tasks that otherwise might go undiscovered during traditional assessment [262]. According to Maceira-Elvira et al. [262] a combination of IMUs and EMG data offers an optimal balance in ease of use, data quality, robustness and obtrusiveness for wearable sensing in stroke rehabilitation. The combination of EMG with kinematic data has shown to improve the classification accuracy compared kinematic data alone [263, 264]. Hand gesture recognition, as an example, might be improved with the fusion of EMG and kinematic data since the finger position changes more significantly with respect to the EMG information during hand movement [263]. Furthermore, EMG can provide information about muscular co-activation and in combination with joint movements over time might provide further insight in joint stiffness and impedance control strategies [265]. The iHams system allows for the integration of kinematic and EMG metrics. The current system only has a limited number of EMG electrodes. Miniaturizing the EMG electrodes allows for high density grids introducing the possibility to extract information at the motor unit level [263].

The third metric of relevance are fingertip interaction forces. Although the performed motor tasks performed by subjects in Chapter 2 are only limited to tasks of the FMA-UE that do not include interaction with physical objects, interaction tasks with such physical objects resemble tasks that are closer to all day activities. The most basic form of kinematic data, i.e. changes in force level, can be used to improve segmentation of the grasping phase. However, more accurate normal and shear force measures during grasp, in combination with kinematic measurements, could increase the insights into movement control and effectiveness of the dynamic interaction with the environment, such as force limitation due to weakness or force overshoot in case of stroke-related deficits [19]. Additionally, shear force components can provide insights into the slip of an object.

Current available sensors for placement on the fingertips provide fine grain information but are relatively bulky and lack compliance to the fingertip tissue eliminating the touch sensation of the user. In addition, most available sensors solutions only provide a one dimensional output. One exception is the 6 -DoF 
force and moment sensor developed by Brookhuis et al. [247]. However, such a stiff sensor appeared not to be applicable for use on the fingertips due to the lack of compliance to fingertip tissue, resulting in poor sensor to skin attachment and the loss of touch sensation [18]. Ideally soft minimal obtrusive sensors on the fingertips should be used to capture these interaction forces such that there are no limitations on the used objects and tasks. The flexible fingertip force sensors presented in Chapter 6 show a high correlation to the applied normal and shear force. Although, these sensors still need to be evaluated for the absolute level of applied force, they could already be used for grasp detection applications. Changes of the applied force during grasp could be used to detect force overshoots caused by stroke related deficits. Furthermore, the shear force captured by these sensors could provide more information such as the influence of gravity on the grasping strategy, slippage and acceleration while handling an object.

The force sensors used and developed in this work only focus on the (limited set of) fingertips. Actual all day grasping, such as handling a cup or a door handle involves interaction areas around the whole hand. To capture these interaction forces, a distributed sensing network placed between the object and the phalanges and hand palm is needed. Glove-like structures equipped with distributed pressure sensors made of fabric have been demonstrated by Bianchi et al. [266]. Additionally, Hammond et al. [250] have shown a pressure sensitive glove based on soft moulded elastomers with embedded wiring and liquid metal for sensing. Both glove structures do however lack the capability to detect shear forces. Another element of these gloves is the ability to capture the hand poses by measuring bending around the finger joints [266, 250]. Christ et al. [87] have demonstrated such a FDM 3D printed glove prototype capable of detecting flexure using piezoresistive sensors. Finger motion tracking using goniometric sensors is established technology and multiple products such as StretchSense MoCap [267] and Xsens Gloves [268] are available on the market. Goniometric sensors can also be used on other body joints to capture joint movements and angles. An example is the knitted piezoresistive fabric goniometer presented by Tognetti et al. [242]. These sensors might eliminate the need for inertial sensors in situations where only joint movements and angles without complete body orientations are sufficient.

The flexibility and compliance of the textile [242, 266] and moulded elastomer structures [250] and the used fabrication methods allow for the fabrication of minimally obtrusive sensors. However, the fabrication methods are quite involved to allow fast fabrication of personalized structures. In future, $3 \mathrm{D}$ printing might be the technology to quickly fabricate distributed interaction 
force sensing structures personalised to the hand of the user.

Wearable sensing systems could be coupled with active arm support systems to automatically adapt the level of needed support [269]. At the moment, the compensation force applied by such a system is set by trial and error by the physical therapist. This results in a variation of the compensation force, leading to non-ideal support and reduction of the effectiveness of the training. Providing a feedback loop to set the level of support based on metrics such as fatigue or compensation strategies, will potentially maximize the patients training effort and duration. Proposed metrics to provide feedback are EMG of the shoulder and arm muscles and the selected kinematic metrics of the arm and trunk. EMG can provide information about pathological muscles coupling between the arm muscles. Furthermore, changes in the spectral characteristics and amplitude of EMG signals correlate with the development of fatigue [270]. Kinematic data can provide information about the reaching distance of the patients arm. This metric is relevant, since the reaching distance of hemiparetic stroke subjects is highly limited with increasing arm load due to the pathological muscles coupling [13]. The endpoint of the arm support does not provide sufficient information since the user is able to perform compensatory trunk movements. A common practice is to immobilize trunk movements by strapping the patient to the chair [8]. However, it is relevant to assess such compensation strategies used by the patient. Furthermore, this method is not desirable in a home-based training setting. As a solution an additional IMU on the patients trunk can be used to detect these compensatory movements, that might be an expression for fatigue or a low level of support. Although training systems that use feedback based on kinematic metrics are technically possible, studies on the feedback methods and metrics are still in a very early state. Future studies are needed to develop a feedback algorithm based on EMG and kinematic data and to evaluate the effectiveness and reliability of these feedback algorithms and metrics during rehabilitation.

The presented body worn integrated sensing system allows for recording outside the clinic. Therefore, these systems enable daily-life monitoring and long term training by the patients in their home-environment. Supplementary studies on the effectiveness metrics of the rehabilitation exercises and on methods to provide feedback to the patient, are expected to result in increased therapy effectiveness based on the patients needs.

Besides applications in the assessment and evaluation of motor function recovery, the data captured with integrated distributed sensing systems is beneficially in many more applications. Reliable movement data in combination with interaction forces and EMG is of high interest for the development of ap- 
plications in robotics, teleoperation, prosthesis and assistive devices [250, 236]. Other applications are to be found in sports [236, 271] and in entertainment such as gaming and animation.

\subsection{Development of 3D printed sensors}

$3 \mathrm{D}$ printing technology is well suited for the production of small volume and customized parts and is already a well-established technology in many hobbyist, prototype and research environments. The increasing availability of new materials with soft, flexible and conductive properties and the ongoing development of $3 \mathrm{D}$ printing sensors makes $3 \mathrm{D}$ printing a perfect candidate for the development of customized sensors for biomedical applications.

The 3D printed EMG electrodes seem to have comparable performance to gold standard $\mathrm{AgCl}$ gel electrodes when electrodes with a diameter of $16 \mathrm{~mm}$ are used. It is unknown how smaller electrodes perform compared to established small electrodes since these studies have not been performed. Smaller printed electrodes seem to have lower performance compared to the larger gold standard $\mathrm{AgCl}$ electrodes with a diameter of $16 \mathrm{~mm}$. However, the outcome is not conclusive due to individual differences between subjects. Smaller electrodes are of interest for the fabrication of high density electrode grids. On the other hand, the need for 3D printed high density electrode grids might be limited since commercial high density grid electrodes could be used to select a reduced set of optimally placed electrodes [234]. Subsequently, these positions can be used as input for a 3D printed personalised EMG electrode structure. Figure 7.1 shows a representative case of the integration of printed sensors in customized structures. In these structures the size of the band and electrode positions are personalised to a specific user. These bands already have been used in combination with gesture recognition algorithms for the control of a robotic hand. Additionally, 3D printed sensors have been used in an integrated system for assisting upper-limb impaired stroke subjects to control a robotics sixth finger using EMG acquired from the frontalis muscle [272]. These initial results indicate that $3 \mathrm{D}$ printed sEMG electrodes have a high potential for local fabrication of personalized sensing structures which can be integrated in e.g. prosthetic and orthotic applications.

The fingertip force sensors presented in this work use carbon doped TPU as piezoresistive sensing material. These materials add many nonlinearities due to the physical changes in the polymer matrix and the viscoelastic properties of the polymer. Low strain values cause alignment of the conductive carbon particles and the formation of new conductive pathways [241] resulting in an 


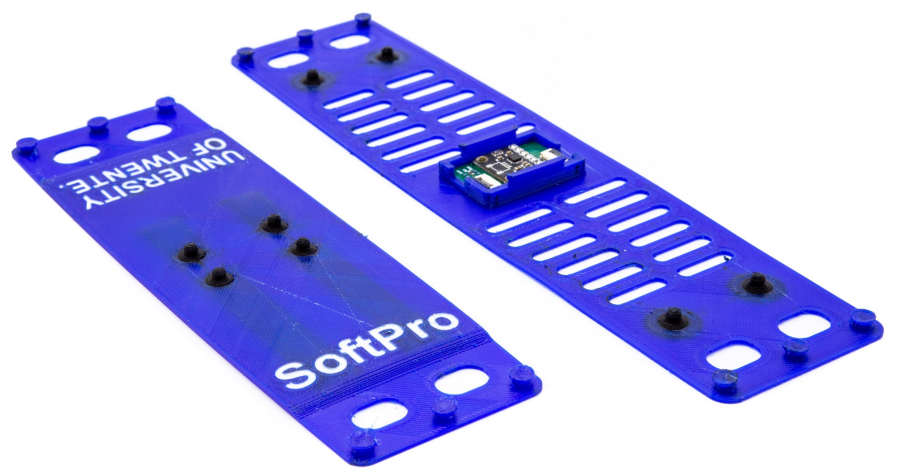

Figure 7.1: 3D printed flexible arm band with integrated EMG electrodes.

initial resistivity drop before the resistivity rises due to breakdown of these networks at higher strain values. Additionally, we hypothesize that the mobility of polymer chains of the filler matrix causes the formation and breakdown of conductive networks as also seen in conductive rubber composites. This causes the resistivity to increase at higher strain rates since the breakdown effect becomes more dominant under these conditions [149, 150]. The hypothesis of formation and breakdown due to polymer mobility is supported by the limited positive temperature coefficient (PTC) of the carbon doped TPU materials. Higher temperatures result in a drop in resistance (NTC) due to increased mobility of the polymer chains resulting in re-connection of the conductive pathways of the filler matrix [273]. Furthermore, heating samples of carbon doped TPU over time periods of hours, also referred to as annealing, results in a significant permanent decline in the resistivity up to a factor of ten.

As an alternative for piezoresistive sensing, 3D printed capacitive sensors have shown their potential $[116,34,186,197,31]$ and are hardly affected by the nonlinear strain-resistance behaviours due to their working principle. However, the mechanical deformation of these sensors still suffers from viscoelastic material behaviours such as hysteresis and softening effects. Piezoresistive sensors, on the other hand, are easier to interface, whereas capacitive sensors require more complex hardware and need proper shielding to limit noise. The relatively low conductivity of carbon-based materials restricts the fabrication of inductors. Due to the ongoing development of printing technologies and materials, high conductive materials are expected that will further stimulate the development of 3D printed inductive sensors and further increase the design freedom of $3 \mathrm{D}$ printed sensor. 
All the work regarding 3D printed sensors in this thesis was based on FDM $3 \mathrm{D}$ printing technologies. Therefore, the materials used for the fabrication of sensors are limited to thermoplastic materials and carbon doped thermoplastics as conductor or functional sensing material. Using hybrid approaches that combine the deposition of thermoplastics and conductive inks or fibers would enable increased conductivity in the structures. FDM 3D printed structures are anisotropic due to the traxel-by-traxel and layer-by-layer fabrication process. Although the anisotropic conduction can be exploited for the development of sensors, in most situation it will limit the design freedom since in most cases a minimum amount of traxel and layer interfaces is desired to achieve low resistance conductive pathways. The relatively low resistivity of conductive inks compared to the resistivity of conductive thermoplastics will consequently allow for longer distance routing in the structures, allowing the fabrication of larger sensing structures. Furthermore, the number of available conductive inks will increase the opportunity to pick the best material for a specific application. Besides, the conductive inks might show less nonlinear behaviour under strain compared to conductive polymer composites.

Although many materials are available for FDM 3D printing, care needs to be taken since the compatibility between materials can be limited due to the differences in chemical nature and/or formulation. Chemical incompatibility or low chemical affinity between materials may reduce the mechanical integrity at the interface of materials in a 3D printed structure [274]. Several types of thermoplastics have differences in thermal properties such as the glass transition temperature and melting temperature, resulting in improper adhesion between traxels of different materials or deformation of parts with a lower glass transition temperature. Additionally, the difference in thermal expansion coefficients results in distortion and/or the formation of cracks and voids between the material interfaces. These issues might be solved by designing mechanical interlocking structures at the interface between materials that have low chemical affinity or dissimilar processing temperatures. These workarounds will however reduce the design freedom offered by the 3D printing process. Depending on the used hardware, FDM 3D printing also has limitation in the number of materials that can be used during one 3D print. Furthermore, switching between these materials still faces challenges in terms of speed, alignment and contamination. FDM 3D printing is also infamous for its rough surface finish and large minimal feature size compared to other additive manufacturing technologies. However, other technologies do at the moment not allow for direct conductive 3D printing and rely on additional post processing steps. In many cases the conductive traces are added by filling channels or cavities with a conductive filler. On first sight, this 
is advantageous since there are a variety of conductive fillers available, allowing to pick the best material for the intended application. However, the high viscosity of many fillers results in large pressure drops and long filling times [223, 83]. Premature solidification, risks for bubble formation and challenges in support material removal [223] all limit the channel length, minimal cross-section and design freedom. The growing interest and ongoing advancements in conductive 3D printing might initiate the development of conductive printing in other 3D printing technologies, e.g. polyjetting or binder jetting.

3D printing show many promises for the fabrication of easy customizable sensing structures, however the technology is not yet mature and there are still many challenges and ongoing research. Fully integrated sensing structures that exploit the full potential of 3D printing are currently limited to only housing electronics, more elaborated sensors are yet to be demonstrated. Many (concept) sensors that have been presented resemble mainly two-dimensional structures, sometimes using the soft properties of the material to flex these structures around three-dimensional surfaces. Many of these structures, such as the EMG electrodes or strain gauges might also be fabricated by using other fabrication methods such as screen- and inkjet-printing conductive inks on flexible substrates $[275,276,277]$. Another option is by using more traditional techniques such as moulding [250] or by using conductive elastic textiles [242, 266]. These methods allow for example easy integration in clothing. It is very likely that the structures fabricated using traditional methods provide more robust signal output and are less subjective to nonlinear effects of the materials. Therefore, it is not likely that at the current state of 3D printed sensing technology, commercial application is already feasible. The future will have to show when or if $3 \mathrm{D}$ printed electronics will defeat traditional methods for the fabrication of customized integrated sensing structures.

3D printing technology has been around since the 1980s. Only in recent years a significant amount of research on 3D printed sensors is performed [81, 82]. These ongoing developments will result in new and improved materials, optimised printing processes and will potentially lead to new sensing principles. This thesis focusses on sensors for biomedical application, although the small volume, customization, integration or distributed fabrication makes 3D printed sensors an interesting technology for many more application areas. One of such is a recent approach in microfluidics and concerns a fully 3D printed thermal mass flow sensor [273]. This sensor utilizes the positive temperature coefficient (PTC) of the carbon doped materials. Although this sensor is relatively large for microfludic applications and has an associated limited bandwidth, the customizability of 3D printing allows the sensors to be tuned to the desired range 
for their application. With the ongoing improvements of 3D printing technology this sensor might in the future be fabricated smaller, increasing its dynamic range. Other applications that can highly benefit from using 3D printed integrated sensors are mechanical components with integrated strain sensing for load measurements [278] or for in situ monitoring of critical mechanical parts [279]. The ongoing advancement in 3D printed soft sensors and the ongoing development of new soft materials will provide new opportunities for integrated sensing applications that will benefit multiple research areas in biomedical and soft robotic [100] sensing applications.

3D printed sensors suffer from many nonlinearities due to the intrinsic properties of the materials used. As shown in Chapter 6, the signal analysis and compensation models are of extreme value to gain a satisfactory sensor response with respect to commercial sensors. 3D printing offers the capability of the creation of integrated complex sensor networks. However, it is not likely that each of these single sensors provide a high quality output. These sensory networks in fact resemble nature's networks of highly nonlinear receptors, like for example the human tactile sensation that originates from many mechanoreceptors in the skin. In our body multiple types of mechanoreceptors have a different sensitivity and respond rapidly or slowly to mechanical stimuli [280]. Interestingly, the mechanical characterization of the carbon doped material as discussed in Chapter 5, shows a strong resemblance with the with the dynamic behaviour of physiological strain sensors in the human skin, as measured using electroneurography (ENG) from the sensory nerve as a result of force stimuli on the skin. Haugland et al. [281] reported that a step force applied to the skin results in a large peak in the ENG response followed by a rapid decline towards a new higher level than before the onset large peak, subsequently the signal slowly decreases during maintained force. At the end of the step force the ENG signal responded again with a sharp positive peak. This pattern shows some similarity with the resistance change of carbon doped TPU under a strain cycle. Furthermore, both the ENG signal and the conductive TPU material show strong hysteretic behaviour. In both cases, this is strongest for the first cycle and continues for subsequent cycles of the hysteresis loop. We therefore might not need to strive for the perfect transducer, but try to resemble nature's networks of highly nonlinear receptors, that in the end translates these chaotic signals to our consciousness of the outside world. 


\section{Chapter 8}

\section{Conclusion}

The goal of this work is the development of a personalized upper limb distributed sensing system to receive continuous information about the interaction of the human body with the environment. This system uses three sensor modalities: kinematic, electromyography (EMG) and kinetic sensing data. Preferably these sensor modalities are integrated into soft and flexible personalized sensing structures.

The distributed inertial measuring system has proven to be a useful tool in the quantitative assessment of the upper extremity motor functions of stroke subjects. The pilot study performed on 10 chronic stroke subjects has shown that these systems are capable of objectively distinguishing postures between the affected and non-affected upper extremities of stroke subjects and assess features related to the pathological synergies that are potentially clinically relevant. Since individual joint angles do not show a clear relation to the FMA-UE sub score, the distributed IMU system has the potential to provide more detailed and different information about the performance of the affected upper extremities of stroke subjects with respect to the FMA-UE sub score. However, the clinical relevance of this information has yet to be demonstrated.

3D printed EMG electrode structures have proven to be a successful application of $3 \mathrm{D}$ printed personalized sensing structures. The high resistance and nonlinear properties of the conductive TPU materials, hampering to some extend force sensing applications, seem not to affect the performance of these electrodes. The 3D printed electrode structures provide equal performance as the gold standard $\mathrm{AgCl}$ gel electrodes in terms of signal amplitude and in practical applications such as gestures recognition for the control of assistive devices.

The development of soft sensors is initiated by the limited usefulness of excising technologies, that are too large and stiff for practical applications on the fingertips. Flexible 3D printed soft fingertips force sensors are still in an early stage of their development and the signal quality does not compete with 
existing force sensors. The output does, however, highly correlate with the applied force when paired with models that compensate the nonlinear effects of the sensing materials. In many situations only grasp detection or just the relative change of force, under for example the influence of shoulder flexion over time, is already of added value. Research on 3D printing of force sensors and other conductive structures has shown many challenges and yielded new insights into the nonlinear materials properties and anisotropic conduction of traxel based 3D printed structures. The commonly used carbon-infused thermoplastic materials in FDM 3D printing have a gauge factor that depends on the amount of strain, that is time- and strain-rate-dependent and this gauge factor can even be negative. The anisotropic conduction causes a high impedance between traxels, especially in the $z$-direction. This effect can be employed and used as sensing principle. These new insights will potentially unfold in improved printing technologies, materials and signal analysis methods to compensate for the high nonlinearity of the sensing structures.

3D printing will allow for easy adaptations, improving the customisability in sensorized structures. The ongoing research on sensor designs, signal analysis methods and the advent of 3D printing technologies, will enable the future fabrication of personalised integrated sensing devices at affordable prices for applications in e.g. prosthetics, orthotics or soft robotics. These structures could also transfer to clinical applications where there is a need for interaction sensing systems that do not impede the user's movements. The development of these systems will improve our knowledge, understanding and assessment of pathological synergies and may eventually enable personalized and targeted rehabilitation activities. 


\section{Bibliography}

[1] W. Johnson, O. Onuma, M. Owolabi, and S. Sachdev, "Stroke: a global response is needed," Bulletin of the World Health Organization, vol. 94, no. 9, pp. 634-634A, sep 2016. [Online]. Available: http: //www.who.int/entity/bulletin/volumes/94/9/16-181636.pdf

[2] C. O. Johnson, et al., "Global, regional, and national burden of stroke, 1990-2016: a systematic analysis for the Global Burden of Disease Study 2016," The Lancet Neurology, vol. 18, no. 5, pp. 439-458, 2019.

[3] J. Mackay and G. A. Mensah, The atlas of heart disease and stroke. World Health Organization, 2004.

[4] P. Langhorne, F. Coupar, and A. Pollock, "Motor recovery after stroke: a systematic review," The Lancet Neurology, vol. 8, no. 8, pp. 741-754, 2009. [Online]. Available: http://dx.doi.org/10.1016/S1474-4422(09)70150-4

[5] T. Krabben, B. I. Molier, A. Houwink, J. S. Rietman, J. H. Buurke, and G. B. Prange, "Circle drawing as evaluative movement task in stroke rehabilitation: an explorative study," Journal of NeuroEngineering and Rehabilitation, vol. 8, no. 1, p. 15, 2011. [Online]. Available: https://doi.org/10.1186/1743-0003-8-15

[6] A. Schwarz, C. M. Kanzler, O. Lambercy, A. R. Luft, and J. M. Veerbeek, "Systematic Review on Kinematic Assessments of Upper Limb Movements After Stroke," Stroke, vol. 50, no. 3, pp. 718-727, 2019.

[7] A. A. Timmermans, H. A. Seelen, R. D. Willmann, and H. Kingma, "Technologyassisted training of arm-hand skills in stroke: Concepts on reacquisition of motor control and therapist guidelines for rehabilitation technology design," Journal of NeuroEngineering and Rehabilitation, vol. 6, no. 1, 2009.

[8] T. M. Sukal, M. D. Ellis, and J. P. Dewald, "Shoulder abduction-induced reductions in reaching work area following hemiparetic stroke: Neuroscientific implications," Experimental Brain Research, vol. 183, no. 2, pp. 215-223, 2007.

[9] M. M. Bhagubai, G. Wolterink, A. Schwarz, J. P. Held, B. J. F. Van Beijnum, and P. H. Veltink, "Quantifying Pathological Synergies in the Upper Extremity of Stroke Subjects with the Use of Inertial Measurement Units: A Pilot Study," IEEE Journal of Translational Engineering in Health and Medicine, vol. 9, no. November 2020, 2020.

[10] G. Kwakkel, N. A. Lannin, K. Borschmann, C. English, M. Ali, L. Churilov, G. Saposnik, C. Winstein, E. E. Van Wegen, S. L. Wolf, J. W. Krakauer, 
and J. Bernhardt, "Standardized Measurement of Sensorimotor Recovery in Stroke Trials: Consensus-Based Core Recommendations from the Stroke Recovery and Rehabilitation Roundtable," Neurorehabilitation and Neural Repair, vol. 31, no. 9, pp. 784-792, 2017.

[11] A. Filippeschi, N. Schmitz, M. Miezal, G. Bleser, E. Ruffaldi, and D. Stricker, "Survey of motion tracking methods based on inertial sensors: A focus on upper limb human motion," Sensors (Switzerland), vol. 17, no. 6, pp. 1-40, 2017.

[12] M. D. Ellis, T. Sukal, T. DeMott, and J. P. A. Dewald, "Augmenting clinical evaluation of hemiparetic arm movement with a laboratory-based quantitative measurement of kinematics as a function of limb loading," Neurorehabilitation and neural repair, vol. 22, no. 4, pp. 321-329, 2008.

[13] M. D. Ellis, Y. Lan, J. Yao, and J. P. Dewald, "Robotic quantification of upper extremity loss of independent joint control or flexion synergy in individuals with hemiparetic stroke: a review of paradigms addressing the effects of shoulder abduction loading," Journal of NeuroEngineering and Rehabilitation, vol. 13, no. 1, pp. 1-11, 2016. [Online]. Available: http://dx.doi.org/10.1186/s12984-016-0203-0

[14] E. Battaglia, M. Bianchi, A. Altobelli, G. Grioli, M. G. Catalano, A. Serio, M. Santello, and A. Bicchi, "ThimbleSense: A Fingertip-Wearable Tactile Sensor for Grasp Analysis," IEEE Transactions on Haptics, vol. 9, no. 1, pp. 121-133, 2016.

[15] R. Parry, S. Macias Soria, P. Pradat-Diehl, V. Marchand-Pauvert, N. Jarrassé, and A. Roby-Brami, "Effects of Hand Configuration on the Grasping, Holding, and Placement of an Instrumented Object in Patients With Hemiparesis," Frontiers in Neurology, vol. 10, no. March, pp. 1-15, 2019.

[16] A. Serio, E. Riccomini, V. Tartaglia, I. Sarakoglou, M. Gabiccini, N. Tsagarakis, and A. Bicchi, "The patched intrinsic tactile object: A tool to investigate human grasps," IEEE International Conference on Intelligent Robots and Systems, no. Iros, pp. 1261-1268, 2014.

[17] M. A. Roa, R. Koiva, and C. Castellini, "Experimental evaluation of human grasps using a sensorized object," Proceedings of the IEEE RAS and EMBS International Conference on Biomedical Robotics and Biomechatronics, pp. 16621668, 2012.

[18] H. G. Kortier, H. M. Schepers, and P. H. Veltink, "Identification of Object Dynamics Using Hand Worn Motion and Force Sensors," Sensors (Basel, Switzerland), vol. 16, no. 12, p. 2005, nov 2016. [Online]. Available: http://www.mdpi.com/1424-8220/16/12/2005

[19] A. Schwarz, M. Bhagubai, G. Wolterink, J. P. O. Held, A. R. Luft, and P. H. Veltink, "Assessment of Upper Limb Movement Impairments after Stroke Using Wearable Inertial Sensing," Sensors, vol. 20, no. 17, p. 4770, 2020. [Online]. Available: https://doi.org/10.3390/s20174770

[20] R. Merletti, A. Botter, A. Troiano, E. Merlo, and M. A. Minetto, "Technology and instrumentation for detection and conditioning of the surface electromyographic signal: State of the art," Clinical Biomechanics, vol. 24, 
no. 2, pp. 122-134, 2009. [Online]. Available: http://dx.doi.org/10.1016/j. clinbiomech.2008.08.006http://www.ncbi.nlm.nih.gov/pubmed/19042063

[21] M. B. Reaz, M. S. Hussain, and F. Mohd-Yasin, "Techniques of EMG signal analysis: detection, processing, classification and applications (Correction)," Biol Proced Online, vol. 8, p. 163, 2006. [Online]. Available: http://www.ncbi.nlm.nih.gov/pubmed/19565309

[22] M. Piotrkiewicz and B. Kuraszkiewicz, "Afterhyperpolarization of human motoneurons firing double and triple discharges," Front Hum Neurosci, vol. 8, p. 373, 2014. [Online]. Available: http://www.ncbi.nlm.nih.gov/pubmed/24910608

[23] R. G. Scalisi, M. Paleari, A. Favetto, M. Stoppa, P. Ariano, P. Pandolfi, and A. Chiolerio, "Inkjet printed flexible electrodes for surface electromyography," Organic Electronics, vol. 18, pp. 89-94, 2015.

[24] J. P. A. Dewald, P. S. Pope, J. D. Given, T. S. Buchanan, and W. Z. Rymer, "Abnormal muscle coactivation patterns during isometric torque generation at the elbow and shoulder in hemiparetic subjects," Brain, vol. 118, no. 2, pp. 495-510, 1995.

[25] J. M. Wagner, A. W. Dromerick, S. A. Sahrmann, and C. E. Lang, "Upper extremity muscle activation during recovery of reaching in subjects with poststroke hemiparesis," Clinical Neurophysiology, vol. 118, no. 1, pp. 164-176, 2007.

[26] J. Chae, G. Yang, B. Park, and I. Labatia, "Muscle Weakness and Cocontraction in Upper Limb Hemiparesis: Relationship to Motor Impairment and Physical Disability," Neurorehabilitation and Neural Repair, vol. 16, no. 3, pp. 241-248, jan 2002.

[27] H. G. Kortier, V. I. Sluiter, D. Roetenberg, and P. H. Veltink, "Assessment of hand kinematics using inertial and magnetic sensors," Journal of NeuroEngineering and Rehabilitation, vol. 11, p. 70, 2014. [Online]. Available: http://www.jneuroengrehab.com/content/11/1/70http: //www.jneuroengrehab.com/content/11/1/70

[28] M. Schouten, G. Wolterink, A. Dijkshoorn, D. Kosmas, S. Stramigioli, and G. Krijnen, "A Review of Extrusion-Based 3D Printing for the Fabrication of Electro-and Biomechanical Sensors," IEEE Sensors Journal, no. AUGUST, pp. $1-12,2020$.

[29] D. Fraser, G. Andy, and R. Adarsh D, "Influence of interface in electrical properties of 3D printed structures," Additive Manufacturing, 2021. [Online]. Available: https://doi.org/10.1016/j.addma.2021.102206

[30] A. Dijkshoorn, M. Schouten, G. Wolterink, R. Sanders, G. Krijnen, S. Stramigioli, and G. Krijnen, "Characterizing the Electrical Properties of Anisotropic, 3D-Printed Conductive Sheets for Sensor Applications," IEEE Sensors Journal, vol. 20, no. 23, pp. 14218-14227, 2020.

[31] G. Wolterink, R. Sanders, and G. Krijnen, "Thin, flexible, capacitive force sensors based on anisotropy in 3D-printed structures," in 2018 IEEE SENSORS, vol. 2018-Octob, IEEE. IEEE, 2018, pp. 1-4.

[32] TMS-International B.V. No Title. [Online]. Available: https://www.tmsi.com/ 
[33] A. Dijkshoorn, J. Cui, S. Stramigioli, and G. Krijnen, "First results of a Soft, 3D-Printed, Resistive Cantilever Flow Sensor," pp. 1-4, 2021.

[34] M. Schouten, R. Sanders, and G. Krijnen, "3D printed flexible capacitive force sensor with a simple micro-controller based readout," Proceedings of IEEE Sensors, vol. 2017-Decem, no. 3, pp. 1-3, 2017.

[35] M. Schouten, B. Prakken, R. Sanders, and G. Krijnen, "Linearisation of a 3D printed flexible tactile sensor based on piezoresistive sensing," Proceedings of IEEE Sensors, vol. 2019-Octob, pp. 2019-2022, 2019.

[36] M. Schouten, C. Spaan, D. Kosmas, R. Sanders, and G. Krijnen, "3d printed capacitive shear and normal force sensor using a highly flexible dielectric," in 2021 IEEE Sensors Applications Symposium (SAS). IEEE, 2021, pp. 1-6.

[37] SoftPro Project 2016. No Title. [Online]. Available: https://www.softpro.eu

[38] Y. Béjot, H. Bailly, J. Durier, and M. Giroud, "Epidemiology of stroke in Europe and trends for the 21st century," La Presse Médicale, vol. 45, no. 12, 2016.

[39] E. J. Benjamin, P. Muntner, A. Alonso, M. S. Bittencourt, C. W. Callaway, A. P. Carson et al., "Heart Disease and Stroke Statistics-2019 Update: A Report From the American Heart Association," Circulation, vol. 139, no. 10, mar 2019. [Online]. Available: https://www.ahajournals.org/doi/10.1161/CIR. 0000000000000659

[40] E. S. Donkor, "Stroke in the21stCentury: A Snapshot of the Burden, Epidemiology, and Quality of Life," Stroke Research and Treatment, vol. 2018, pp. 1-10, 2018 .

[41] S. M. Hatem, G. Saussez, M. D. Faille, V. Prist, X. Zhang, D. Dispa, and Y. Bleyenheuft, "Rehabilitation of Motor Function after Stroke: A Multiple Systematic Review Focused on Techniques to Stimulate Upper Extremity Recovery," Frontiers in Human Neuroscience, vol. 10, 2016.

[42] P. Raghavan, "Upper Limb Motor Impairment After Stroke," Physical Medicine and Rehabilitation Clinics of North America, vol. 26, no. 4, pp. 599-610, 2015. [Online]. Available: http://dx.doi.org/10.1016/j.pmr.2015.06.008

[43] A. J. McMorland, K. D. Runnalls, and W. D. Byblow, "A neuroanatomical framework for upper limb synergies after stroke," Frontiers in Human Neuroscience, vol. 9, no. FEB, pp. 1-6, 2015.

[44] J. van Kordelaar, E. E. H. van Wegen, and G. Kwakkel, "Unraveling the interaction between pathological upper limb synergies and compensatory trunk movements during reach-to-grasp after stroke: a cross-sectional study," Experimental brain research, vol. 221, no. 3, pp. 251-262, 2012.

[45] T. E. Twitchell, "The Restoration Of Motor Function Following Hemiplegia In Man," Brain, vol. 74, no. 4, pp. 443-480, 1951.

[46] S. Brunnstrom, "Motor Testing Procedures in Hemiplegia: Based on Sequential Recovery Stages," Physical Therapy, vol. 46, no. 4, pp. 357-375, jan 1966.

[47] J. P. A. Dewald and R. F. Beer, "Abnormal joint torque patterns in the paretic upper limb of subjects with hemiparesis," Muscle 8 Nerve: Official Journal of the American Association of Electrodiagnostic Medicine, vol. 24, no. 2, pp. 273-283, 2001. 
[48] M. C. Cirstea and M. F. Levin, "Compensatory strategies for reaching in stroke," Brain, vol. 123, no. 5, pp. 940-953, 2000.

[49] C. E. Lang, M. D. Bland, R. R. Bailey, S. Y. Schaefer, and R. L. Birkenmeier, "Assessment of upper extremity impairment, function, and activity after stroke: Foundations for clinical decision making," Journal of Hand Therapy, vol. 26, no. 2, pp. 104-115, 2013. [Online]. Available: http://dx.doi.org/10.1016/j.jht.2012.06.005

[50] L. Santisteban, M. Térémetz, J.-P. Bleton, J.-C. Baron, M. A. Maier, and P. G. Lindberg, "Upper Limb Outcome Measures Used in Stroke Rehabilitation Studies: A Systematic Literature Review," Plos One, vol. 11, no. 5, jun 2016.

[51] A. R. Fugl-Meyer, L. Jääskö, I. Leyman, S. Olsson, and S. Steglind, "The poststroke hemiplegic patient. 1. a method for evaluation of physical performance," Scandinavian journal of rehabilitation medicine, vol. 7, no. 1, pp. 13-31, 1975. [Online]. Available: http://europepmc.org/abstract/MED/1135616

[52] D. J. Gladstone, C. J. Danells, and S. E. Black, "The Fugl-Meyer Assessment of Motor Recovery after Stroke: A Critical Review of Its Measurement Properties," Neurorehabilitation and Neural Repair, vol. 16, no. 3, pp. 232-240, 2002.

[53] A. D. L. Reyes-Guzmán, I. Dimbwadyo-Terrer, F. Trincado-Alonso, F. Monasterio-Huelin, D. Torricelli, and A. Gil-Agudo, "Quantitative assessment based on kinematic measures of functional impairments during upper extremity movements: A review," Clinical Biomechanics, vol. 29, no. 7, pp. 719-727, 2014.

[54] M. A. Murphy, C. Willén, and K. S. Sunnerhagen, "Kinematic Variables Quantifying Upper-Extremity Performance After Stroke During Reaching and Drinking From a Glass," Neurorehabilitation and Neural Repair, vol. 25, no. 1, pp. 71-80, sep 2010.

[55] G. Gera, S. M. S. F. Freitas, and J. P. Scholz, "Relationship of diminished interjoint coordination after stroke to hand path consistency," Experimental Brain Research, vol. 234, no. 3, pp. 741-751, 2015.

[56] G. Thrane, M. A. Murphy, and K. S. Sunnerhagen, "Recovery of kinematic arm function in well-performing people with subacute stroke: a longitudinal cohort study," Journal of NeuroEngineering and Rehabilitation, vol. 15, no. 1, 2018.

[57] A. Frisoli, E. Sotgiu, C. Procopio, M. Bergamasco, C. Chisari, G. Lamola, and B. Rossi, "Training and assessment of upper limb motor function with a robotic exoskeleton after stroke," 2012 4th IEEE RAS and EMBS International Conference on Biomedical Robotics and Biomechatronics, 2012.

[58] A. M. Coderre, A. A. Zeid, S. P. Dukelow, M. J. Demmer, K. D. Moore, M. J. Demers, H. Bretzke, T. M. Herter, J. I. Glasgow, K. E. Norman, and E. al., "Assessment of Upper-Limb Sensorimotor Function of Subacute Stroke Patients Using Visually Guided Reaching," Neurorehabilitation and Neural Repair, vol. 24, no. 6 , pp. 528-541, 2010.

[59] S. Mazzoleni, M. C. Carrozza, P. Sale, M. Franceschini, F. Posteraro, and M. Tiboni, "Effects of upper limb robot-assisted therapy on motor recovery of subacute stroke patients: A kinematic approach," 2013 IEEE 13th International Conference on Rehabilitation Robotics (ICORR), 2013. 
[60] C. P. Walmsley, S. A. Williams, T. Grisbrook, C. Elliott, C. Imms, and A. Campbell, "Measurement of Upper Limb Range of Motion Using Wearable Sensors: A Systematic Review," Sports Medicine - Open, vol. 4, no. 1, 2018.

[61] J. P. O. Held, B. Klaassen, A. Eenhoorn, B.-J. F. van Beijnum, J. H. Buurke, P. H. Veltink, and A. R. Luft, "Inertial sensor measurements of upper-limb kinematics in stroke patients in clinic and home environment," Frontiers in bioengineering and biotechnology, vol. 6, p. 27, 2018.

[62] Y. Li, X. Zhang, Y. Gong, Y. Cheng, X. Gao, and X. Chen, "Motor Function Evaluation of Hemiplegic Upper-Extremities Using Data Fusion from Wearable Inertial and Surface EMG Sensors," Sensors, vol. 17, no. 3, p. 582, 2017.

[63] H. G. Kortier, V. I. Sluiter, D. Roetenberg, and P. H. Veltink, "Assessment of hand kinematics using inertial and magnetic sensors," Journal of NeuroEngineering and Rehabilitation, vol. 11, no. 1, p. 70, 2014.

[64] H. Luinge, P. Veltink, and C. Baten, "Ambulatory measurement of arm orientation," Journal of Biomechanics, vol. 40, no. 1, pp. 78-85, 2007.

[65] L. Ricci, D. Formica, L. Sparaci, F. Lasorsa, F. Taffoni, E. Tamilia, and E. Guglielmelli, "A New Calibration Methodology for Thorax and Upper Limbs Motion Capture in Children Using Magneto and Inertial Sensors," Sensors, vol. 14, no. 1, pp. 1057-1072, 2014.

[66] W. Kong, S. Sessa, M. Zecca, and A. Takanishi, "Anatomical Calibration through Post-Processing of Standard Motion Tests Data," Sensors, vol. 16, no. 12, p. 2011, 2016.

[67] S. O. H. Madgwick, A. J. L. Harrison, and R. Vaidyanathan, "Estimation of IMU and MARG orientation using a gradient descent algorithm," 2011 IEEE International Conference on Rehabilitation Robotics, 2011.

[68] B. Kirking, M. El-Gohary, and Y. Kwon, "The feasibility of shoulder motion tracking during activities of daily living using inertial measurement units," Gait and Posture, vol. 49, pp. 47-53, 2016.

[69] J. B. Kuipers, Quaternions and rotation sequences: a primer with applications to orbits, aerospace, and virtual reality. Princeton University Press, 2007.

[70] Rehabilitation Medicine, University of Gothenburg. (2019) Fugl-Meyer Assessment Upper Extremity (FMA-UE), Assessment of sensorimotor function.

[71] M. A. Murphy, C. Willén, and K. S. Sunnerhagen, "Responsiveness of Upper Extremity Kinematic Measures and Clinical Improvement During the First Three Months After Stroke," Neurorehabilitation and Neural Repair, vol. 27, no. 9, pp. 844-853, 2013.

[72] J. Li, B. Pan, T. Jin, Z. Huang, S. Ye, J. Wu, Z. Huang, B. Xie, C. Luo, C. Wang, and E. al., "A single task assessment system of upper-limb motor function after stroke," Technology and Health Care, vol. 24, no. s2, 2016.

[73] M. L. Woodbury, C. A. Velozo, L. G. Richards, and P. W. Duncan, "Rasch analysis staging methodology to classify upper extremity movement impairment after stroke," Archives of physical medicine and rehabilitation, vol. 94, no. 8, pp. 1527-1533, 2013. 
[74] E.-L. Bustrén, K. S. Sunnerhagen, and M. A. Murphy, "Movement Kinematics of the Ipsilesional Upper Extremity in Persons With Moderate or Mild Stroke," Neurorehabilitation and Neural Repair, vol. 31, no. 4, pp. 376-386, 2017.

[75] S. K. Subramanian, J. Yamanaka, G. Chilingaryan, and M. F. Levin, "Validity of Movement Pattern Kinematics as Measures of Arm Motor Impairment Poststroke," Stroke, vol. 41, no. 10, pp. 2303-2308, 2010.

[76] C. Duret, O. Courtial, and A. G. Grosmaire, "Kinematic measures for upper limb motor assessment during robot-mediated training in patients with severe sub-acute stroke," Restorative Neurology and Neuroscience, vol. 34, no. 2, pp. 237-245, 2016.

[77] M. A. Murphy and C. K. Häger, "Kinematic analysis of the upper extremity after stroke - how far have we reached and what have we grasped?" Physical Therapy Reviews, vol. 20, no. 3, pp. 137-155, 2015.

[78] C. Weller, R. Kleer, and F. T. Piller, "Economic implications of 3D printing: Market structure models in light of additive manufacturing revisited," International Journal of Production Economics, vol. 164, pp. 43-56, 2015. [Online]. Available: http://dx.doi.org/10.1016/j.ijpe.2015.02.020

[79] T. Pereira, J. V. Kennedy, and J. Potgieter, "A comparison of traditional manufacturing vs additive manufacturing, the best method for the job," Procedia Manufacturing, vol. 30, pp. 11-18, 2019. [Online]. Available: https://doi.org/10.1016/j.promfg.2019.02.003

[80] M. Attaran, "The rise of 3-D printing: The advantages of additive manufacturing over traditional manufacturing," Business Horizons, vol. 60, no. 5, pp. 677-688, 2017. [Online]. Available: http://dx.doi.org/10.1016/j.bushor.2017.05.011

[81] Y. Xu, X. Wu, X. Guo, B. Kong, M. Zhang, X. Qian, S. Mi, and W. Sun, "The Boom in 3D-Printed Sensor Technology," Sensors (Basel, Switzerland), vol. 17, no. 5, pp. 1-37, 2017.

[82] M. R. Khosravani and T. Reinicke, "3D-printed sensors: Current progress and future challenges," Sensors and Actuators, A: Physical, vol. 305, p. 111916, 2020. [Online]. Available: https://doi.org/10.1016/j.sna.2020.111916

[83] A. Dijkshoorn, P. Werkman, M. Welleweerd, G. Wolterink, B. Eijking, J. Delamare, R. Sanders, and G. J. M. Krijnen, "Embedded sensing: Integrating sensors in 3-D printed structures," Journal of Sensors and Sensor Systems, vol. 7, no. 1, pp. 169-181, 2018.

[84] O. Stř́iteský, J. Průša, and M. Bach, Basics of $3 D$ Printing with Josef Prusa. Prusa Research s.r.o., 2019. [Online]. Available: https: //www.prusa3d.com/ebook-basics-of-3d-printing-with-josef-prusa/

[85] B. Redwoon, F. Schöffer, and B. Garret, The 3D printing handbook. Coers \& Roest, 2017.

[86] A. Manero, P. Smith, J. Sparkman, M. Dombrowski, D. Courbin, A. Kester, I. Womack, and A. Chi, "Implementation of 3D printing technology in the field of prosthetics: Past, present, and future," International Journal of Environmental Research and Public Health, vol. 16, no. 9, 2019. 
[87] J. F. Christ, N. Aliheidari, P. Pötschke, and A. Ameli, "Bidirectional and stretchable piezoresistive sensors enabled by multimaterial 3D printing of carbon nanotube/thermoplastic polyurethane nanocomposites," Polymers, vol. 11, no. 1, 2018. [Online]. Available: https://doi.org/10.3390/polym11010011

[88] K. Kim, J. Park, J. hoon Suh, M. Kim, Y. Jeong, and I. Park, "3D printing of multiaxial force sensors using carbon nanotube (CNT)/thermoplastic polyurethane (TPU) filaments," Sensors and Actuators, A: Physical, vol. 263, pp. 493-500, 2017. [Online]. Available: http://dx.doi.org/10.1016/j.sna.2017. 07.020

[89] B. Eijking, R. Sanders, and G. Krijnen, "Development of whisker inspired 3D multi-material printed flexible tactile sensors," Proceedings of IEEE Sensors, vol. 2017-Decem, pp. 1-3, 2017.

[90] G. Wolterink, P. Dias, R. G. Sanders, F. Muijzer, B. J. van Beijnum, P. Veltink, and G. Krijnen, "Development of soft semg sensing structures using 3d-printing technologies," Sensors (Switzerland), vol. 20, no. 15, pp. 1-19, 2020.

[91] G. Wolterink, R. Sanders, and G. Krijnen, "A flexible, three material, 3Dprinted, shear force sensor for use on finger tips," Proceedings of IEEE Sensors, vol. 2019-Octob, pp. 2-5, 2019.

[92] C. J. Hohimer, G. Petrossian, A. Ameli, C. Mo, and P. Pötschke, "3D Printed Conductive Thermoplastic Polyurethane/Carbon Nanotube Composites for Capacitive and Piezoresistive Sensing in Soft Pneumatic Actuators," Additive Manufacturing, p. 101281, 2020.

[93] S. Krachunov and A. J. Casson, "3D Printed Dry EEG Electrodes," Sensors (Basel, Switzerland), vol. 16, no. 10, 2016.

[94] S. Z. Guo, K. Qiu, F. Meng, S. H. Park, and M. C. McAlpine, "3D Printed Stretchable Tactile Sensors," Advanced Materials, vol. 29, no. 27, pp. 1-8, 2017.

[95] T. J. Wallin, J. Pikul, and R. F. Shepherd, "3D printing of soft robotic systems," Nature Reviews Materials, vol. 3, no. 6, pp. 84-100, 2018. [Online]. Available: http://dx.doi.org/10.1038/s41578-018-0002-2

[96] A. Zolfagharian, A. Kaynak, and A. Kouzani, "Closed-loop 4D-printed soft robots," Materials And Design, vol. 188, p. 108411, 2020. [Online]. Available: $\quad$ https://linkinghub.elsevier.com/retrieve/pii/S0264127519308494https: //doi.org/10.1016/j.matdes.2019.108411

[97] S. Li, H. Zhao, and R. F. Shepherd, "Flexible and stretchable sensors for fluidic elastomer actuated soft robots," MRS Bulletin, vol. 42, no. 2, pp. 138-142, 2017.

[98] S. Mousavi, D. Howard, F. Zhang, J. Leng, and C. H. Wang, "Direct 3D Printing of Highly Anisotropic, Flexible, Constriction-Resistive Sensors for Multidirectional Proprioception in Soft Robots," ACS Applied Materials 85 Interfaces, vol. 12, no. 13, pp. 15631-15643, 2020.

[99] G. Stano and G. Percoco, "Design , 3D printing and characterization of a soft actuator with embedded strain sensor," 2020 IEEE International Symposium on Medical Measurements and Applications (MeMeA), pp. 5-10, 2020. 
[100] K. Elgeneidy, G. Neumann, M. Jackson, and N. Lohse, "Directly printable flexible strain sensors for bending and contact feedback of soft actuators," Frontiers Robotics AI, vol. 5, no. FEB, pp. 1-14, 2018.

[101] B. Shih, C. Christianson, K. Gillespie, S. Lee, J. Mayeda, Z. Huo, and M. T. Tolley, "Design considerations for 3D printed, soft, multimaterial resistive sensors for soft robotics," Frontiers Robotics AI, vol. 6, no. APR, pp. 1-12, 2019.

[102] Y. Yang, Y. Chen, Y. Li, Z. Wang, and Y. Li, "Novel Variable-Stiffness Robotic Fingers with Built-In Position Feedback," Soft Robotics, vol. 4, no. 4, pp. 338$352,2017$.

[103] I. Gibson, D. Rosen, and B. Stucker, Directed Energy Deposition Processes. In: Additive Manufacturing Technologies, 2015.

[104] Capricorn Premium Bowden Tubing, "Capricorn Premium PTFE Bowden Tubing." [Online]. Available: https://www.captubes.com/

[105] M. A. Skylar-Scott, S. Gunasekaran, and J. A. Lewis, "Laser-assisted direct ink writing of planar and 3D metal architectures," Proceedings of the National Academy of Sciences of the United States of America, vol. 113, no. 22, pp. 6137$6142,2016$.

[106] C. F. Revelo and H. A. Colorado, "3D printing of kaolinite clay ceramics using the Direct Ink Writing (DIW) technique," Ceramics International, vol. 44, no. 5, pp. 5673-5682, 2018. [Online]. Available: http://dx.doi.org/10.1016/j.ceramint.2017.12.219

[107] M. Rosenthal, C. Henneberger, A. Gutkes, and C. T. Bues, "Liquid Deposition Modeling: a promising approach for 3D printing of wood," European Journal of Wood and Wood Products, vol. 76, no. 2, pp. 797-799, 2018.

[108] S. M. Kabir, K. Mathur, and A. F. M. Seyam, "A critical review on 3D printed continuous fiber-reinforced composites: History, mechanism, materials and properties," Composite Structures, vol. 232, no. August 2019, pp. 1-24, 2020.

[109] X. Yao, C. Luan, D. Zhang, L. Lan, and J. Fu, "Evaluation of carbon fiber-embedded 3D printed structures for strengthening and structural-health monitoring," Materials and Design, vol. 114, pp. 424-432, 2017. [Online]. Available: http://dx.doi.org/10.1016/j.matdes.2016.10.078

[110] S. Swaminathan, K. B. Ozutemiz, C. Majidi, and S. E. Hudson, "FiberWire: Embedding Electronic Function into 3D Printed Mechanically Strong, Lightweight Carbon Fiber Composite Objects," Proceedings of the 2019 CHI Conference on Human Factors in Computing Systems CHI '19, no. Figure 1, pp. 1-11, 2019. [Online]. Available: http: //dl.acm.org/citation.cfm?doid=3290605.3300797

[111] C. Shemelya, L. Banuelos-Chacon, A. Melendez, C. Kief, D. Espalin, R. Wicker, G. Krijnen, and E. Macdonald, "Multi-functional 3D printed and embedded sensors for satellite qualification structures," 2015 IEEE SENSORS - Proceedings, pp. 1422-1425, 2015.

[112] Z. Wang, C. Luan, G. Liao, X. Yao, and J. Fu, "Mechanical and self-monitoring behaviors of $3 \mathrm{D}$ printing smart continuous carbon fiber-thermoplastic lattice 
truss sandwich structure," Composites Part B: Engineering, vol. 176, no. April, p. 107215, 2019. [Online]. Available: https://doi.org/10.1016/j.compositesb. 2019.107215

[113] C. Luan, X. Yao, C. Liu, L. Lan, and J. Fu, "Self-monitoring continuous carbon fiber reinforced thermoplastic based on dual-material three-dimensional printing integration process," Carbon, vol. 140, pp. 100-111, 2018. [Online]. Available: https://doi.org/10.1016/j.carbon.2018.08.019

[114] Brightlands Materials Center, "Brightlands Materials Center Develops Self-Sensing in 3D Printed Fiber Reinforced Thermoplastics." [Online]. Available: https://www.brightlandsmaterialscenter.com/ self-sensing-in-3d-printed-thermoplastic-composites/

[115] M. Saari, B. Cox, E. Richer, P. S. Krueger, and A. L. Cohen, "Fiber encapsulation additive manufacturing: An enabling technology for 3D printing of electromechanical devices and robotic components," $3 D$ Printing and Additive Manufacturing, vol. 2, no. 1, pp. 32-39, 2015.

[116] M. Saari, B. Xia, B. Cox, P. S. Krueger, A. L. Cohen, and E. Richer, "Fabrication and Analysis of a Composite 3D Printed Capacitive Force Sensor," 3D Printing and Additive Manufacturing, vol. 3, no. 3, pp. 137-141, 2016.

[117] C. Shemelya, F. Cedillos, E. Aguilera, D. Espalin, D. Muse, R. Wicker, and E. Macdonald, "Encapsulated copper wire and copper mesh capacitive sensing for 3-D printing applications," IEEE Sensors Journal, vol. 15, no. 2, pp. 12801286, 2015.

[118] E. Aguilera, J. Ramos, D. Espalin, F. Cedillos, D. Muse, R. Wicker, and E. MacDonald, "3D printing of electro mechanical systems," 24th International SFF Symposium - An Additive Manufacturing Conference, SFF 2013, no. January, pp. 950-961, 2013.

[119] D. Espalin, D. W. Muse, E. MacDonald, and R. B. Wicker, "3D Printing multifunctionality: Structures with electronics," International Journal of Advanced Manufacturing Technology, vol. 72, no. 5-8, pp. 963-978, 2014.

[120] L. G. Blok, M. L. Longana, H. Yu, and B. K. S. Woods, "An investigation into 3D printing of fibre reinforced thermoplastic composites," Additive Manufacturing, vol. 22, no. April, pp. 176-186, 2018.

[121] D. Pascale and I. Simion, "Multi-Material 3D Printer Extruder Concept," Journal of Industrial Design and Engineering Graphics, vol. 13, no. 1, pp. 25-28, 2018.

[122] H. Yuk and X. Zhao, "A New 3D Printing Strategy by Harnessing Deformation, Instability, and Fracture of Viscoelastic Inks," Advanced Materials, vol. 30, no. 6, pp. 1-8, 2018.

[123] Anisoprint Sarl, "CCF\&CBF." [Online]. Available: https://anisoprint.com/ product-cf

[124] J. F. Christ, N. Aliheidari, A. Ameli, and P. Pötschke, "3D printed highly elastic strain sensors of multiwalled carbon nanotube/thermoplastic polyurethane nanocomposites," Materials and Design, vol. 131, pp. 394-401, 2017. 
[125] S. W. Kwok, K. H. H. Goh, Z. D. Tan, S. T. M. Tan, W. W. Tjiu, J. Y. Soh, Z. J. G. Ng, Y. Z. Chan, H. K. Hui, and K. E. J. Goh, "Electrically conductive filament for 3D-printed circuits and sensors," pp. 167-175, 2017.

[126] P. F. Flowers, C. Reyes, S. Ye, M. J. Kim, and B. J. Wiley, "3D printing electronic components and circuits with conductive thermoplastic filament," Additive Manufacturing, vol. 18, pp. 156-163, 2017.

[127] S. F. A. Acquah, B. E. Leonhardt, M. S. Nowotarski, J. M. Magi, K. A. Chambliss, T. E. S. Venzel, S. D. Delekar, and L. A. Al-Hariri, "Carbon Nanotubes and Graphene as Additives in 3D Printing," Carbon Nanotubes - Current Progress of their Polymer Composites, 2016.

[128] L. Flandin, A. Hiltner, and E. Baer, "Interrelationships between electrical and mechanical properties of a carbon black-filled ethylene-octene elastomer," Polymer, vol. 42, no. 2, pp. 827-838, 2001.

[129] F. Gubbels, S. Blacher, E. Vanlathem, R. Jérôme, R. Deltour, F. Brouers, and P. Teyssié, "Design of Electrical Conductive Composites: Key Role of the Morphology on the Electrical Properties of Carbon Black Filled Polymer Blends," Macromolecules, vol. 28, no. 5, pp. 1559-1566, 1995.

[130] S. H. Munson-Mcgee, "Estimation of the critical concentration in an anisotropic percolation network," Physical Review B, vol. 43, no. 4, pp. 3331-3336, 1991.

[131] A. D. Valentine, T. A. Busbee, J. W. Boley, J. R. Raney, A. Chortos, A. Kotikian, J. D. Berrigan, M. F. Durstock, and J. A. Lewis, "Hybrid 3D Printing of Soft Electronics," Advanced Materials, vol. 29, no. 40, pp. 1-8, 2017.

[132] ProtoPlant, makers of Proto-pasta. Composite PLA - Electrically Conductive Graphite. [Online]. Available: https://www.proto-pasta.com/

[133] Graphene 3D Lab. Conductive Graphene PLA Filament. [Online]. Available: http://www.blackmagic3d.com/Conductive-p/grphn-pla.htm

[134] Graphene 3D Lab Inc., "Material Safety Data Sheet." [Online]. Available: http://graphenelab.com/pdf/conductivemsds.pdf

[135] Multi3D. Electrifi Conductive Filament. [Online]. Available: https://www. multi3dllc.com/product/electrifi/

[136] Palmiga Innovation, "PI-ETPU 95-250 Carbon Black." [Online]. Available: https://rubber3dprinting.com/pi-etpu-95-250-carbon-black/

[137] Fenner Drives. Ninjatek.com. [Online]. Available: https://ninjatek.com

[138] Addnorth, "Koltron G1." [Online]. Available: https://addnorth.com/product/ KoltronG1/KoltronG1

[139] H. Kipphan, Handbook of Print Media, 2001, vol. 13, no. 6.

[140] Z. Zhu, H. Wang, and D. Peng, "Dependence of sediment suspension viscosity on solid concentration: A simple general equation," Water (Switzerland), vol. 9, no. $7,2017$.

[141] H. Yuk, B. Lu, S. Lin, K. Qu, J. Xu, J. Luo, and X. Zhao, "3D printing of conducting polymers," Nature Communications, vol. 11, no. 1, pp. 4-11, 2020. [Online]. Available: http://dx.doi.org/10.1038/s41467-020-15316-7 
[142] D. N. Heo, S. J. Lee, R. Timsina, X. Qiu, N. J. Castro, and L. G. Zhang, "Development of 3D printable conductive hydrogel with crystallized PEDOT:PSS for neural tissue engineering," Materials Science and Engineering $C$, vol. 99, no. September 2018, pp. 582-590, 2019. [Online]. Available: https://doi.org/10.1016/j.msec.2019.02.008

[143] F. Antonov, "CONTINUOUS FIBER 3D PRINTING: CURRENT MARKET REVIEW BY FEDOR ANTONOV, CEO ANISOPRINT," 2019. [Online]. Available: https://3dprintingindustry.com/ news/continuous-fiber-3d-printing-current-market-review-by-fedor-antonov\% 5C-ceo-anisoprint-164964/

[144] C. Votzke, U. Daalkhaijav, Y. Menguc, and M. L. Johnston, "3D-Printed Liquid Metal Interconnects for Stretchable Electronics," IEEE Sensors Journal, vol. 19, no. 10 , pp. 3832-3840, 2019.

[145] J. W. Boley, E. L. White, G. T. C. Chiu, and R. K. Kramer, "Direct writing of gallium-indium alloy for stretchable electronics," Advanced Functional Materials, vol. 24, no. 23, pp. 3501-3507, 2014.

[146] H. Ota, S. Emaminejad, Y. Gao, A. Zhao, E. Wu, S. Challa, K. Chen, H. M. Fahad, A. K. Jha, D. Kiriya, W. Gao, H. Shiraki, K. Morioka, A. R. Ferguson, K. E. Healy, R. W. Davis, and A. Javey, "Application of 3D Printing for Smart Objects with Embedded Electronic Sensors and Systems," Advanced Materials Technologies, vol. 1, no. 1, pp. 1-8, 2016.

[147] S. Liu, K. Sweatman, S. McDonald, and K. Nogita, "Ga-based alloys in microelectronic interconnects: A review," Materials, vol. 11, no. 8, pp. 1-20, 2018.

[148] Y. Cui, Y. Ding, S. Xu, Z. Yang, P. Zhang, W. Rao, and J. Liu, "Liquid Metal Corrosion Effects on Conventional Metallic Alloys Exposed to Eutectic Gallium-Indium Alloy Under Various Temperature States," International Journal of Thermophysics, vol. 39, no. 10, pp. 1-14, 2018. [Online]. Available: https://doi.org/10.1007/s10765-018-2440-x

[149] N. C. Das, T. K. Chaki, and D. Khastgir, "Effect of axial stretching on electrical resistivity of short carbon fibre and carbon black filled conductive rubber composites," Polymer International, vol. 51, no. 2, pp. 156-163, 2002.

[150] K. P. Sau, T. K. Chaki, and D. Khastgir, "The effect of compressive strain and stress on electrical conductivity of conductive rubber composites," Rubber Chemistry and Technology, vol. 73, no. 2, pp. 310-324, 2000.

[151] H. J. Qi and M. C. Boyce, "Stress-strain behavior of thermoplastic polyurethanes," Mechanics of Materials, vol. 37, no. 8, pp. 817-839, 2005.

[152] H. Nassar, M. Ntagios, W. T. Navaraj, R. Dahiya, and R. Dahiva, "MultiMaterial 3D Printed Bendable Smart Sensing Structures," Proceedings of IEEE Sensors, vol. 2018-Octob, pp. 3-6, 2018.

[153] U. Daalkhaijav, O. D. Yirmibesoglu, S. Walker, and Y. Mengüç, "Rheological Modification of Liquid Metal for Additive Manufacturing of Stretchable Electronics," Advanced Materials Technologies, vol. 3, no. 4, pp. 1-9, 2018. 
[154] Y. L. Park, B. R. Chen, and R. J. Wood, "Design and fabrication of soft artificial skin using embedded microchannels and liquid conductors," IEEE Sensors Journal, vol. 12, no. 8, pp. 2711-2718, 2012.

[155] H. Kim, F. Torres, Y. Wu, D. Villagran, Y. Lin, and T.-L. Tseng, "Integrated $3 \mathrm{D}$ printing and corona poling process of PVDF piezoelectric films for pressure sensor application," Smart Materials and Structures, vol. 26, no. 8, p. 85027, 2017.

[156] H. Kim, F. Torres, D. Villagran, C. Stewart, Y. Lin, and T. L. B. Tseng, "3D Printing of BaTiO3/PVDF Composites with Electric In Situ Poling for Pressure Sensor Applications," Macromolecular Materials and Engineering, vol. 302, no. 11, pp. 1-6, 2017.

[157] S. Rajala, M. Schouten, G. Krijnen, and S. Tuukkanen, "High Bending-Mode Sensitivity of Printed Piezoelectric Poly(vinylidenefluoride- co-trifluoroethylene) Sensors," ACS Omega, vol. 3, no. 7, pp. 8067-8073, 2018.

[158] S. Bodkhe, P. S. M. Rajesh, F. P. Gosselin, and D. Therriault, "Simultaneous 3D Printing and Poling of PVDF and Its Nanocomposites," ACS Applied Energy Materials, vol. 1, no. 6, pp. 2474-2482, 2018.

[159] E. R. Cholleti, "A Review on 3D printing of piezoelectric materials," IOP Conference Series: Materials Science and Engineering, vol. 455, no. 1, 2018.

[160] C. Lee and J. A. Tarbutton, "Electric Poling-assisted Additive Manufacturing Process for Lead-free Piezoelectric Device Fabrication," Procedia Manufacturing, vol. 1, pp. 320-326, 2015. [Online]. Available: http://dx.doi.org/10.1016/j. promfg.2015.09.035

[161] I. Kierzewski, S. S. Bedair, B. Hanrahan, H. Tsang, L. Hu, and N. Lazarus, "Adding an electroactive response to 3D printed materials: Printing a piezoelectret," Additive Manufacturing, vol. 31, no. July 2019, p. 100963, 2020. [Online]. Available: https://doi.org/10.1016/j.addma.2019.100963

[162] Proto-pasta, "Iron-filled Metal Composite PLA." [Online]. Available: https: //www.proto-pasta.com/products/magnetic-iron-pla

[163] M. bonn, "Neofer 25/60p." [Online]. Available: https://magnetfabrik. $\mathrm{de} / \mathrm{wp}$-content/uploads/produkte/material-list-according-to-din/refeb/ neofer-25-60p.pdf

[164] C. Huber, C. Abert, F. Bruckner, M. Groenefeld, O. Muthsam, S. Schuschnigg, K. Sirak, R. Thanhoffer, I. Teliban, C. Vogler, R. Windl, and D. Suess, "3D print of polymer bonded rare-earth magnets, and 3D magnetic field scanning with an end-user 3D printer," Applied Physics Letters, vol. 109, no. 16, 2016. [Online]. Available: http://dx.doi.org/10.1063/1.4964856

[165] Z. Wang, C. Huber, J. Hu, J. He, D. Suess, and S. X. Wang, "An electrodynamic energy harvester with a $3 \mathrm{D}$ printed magnet and optimized topology," Applied Physics Letters, vol. 114, no. 1, 2019. [Online]. Available: http://dx.doi.org/10.1063/1.5074123

[166] R. Wagner, L. Brandl, W. Kersten, S. Sponar, Y. Hasegawa, C. Huber, F. Bruckner, and D. Suess, "3D printed magnets for neutron spin manipulation," EPJ Web of Conferences, vol. 219, p. 10008, 2019. 
[167] L. Li, A. Tirado, I. C. Nlebedim, O. Rios, B. Post, V. Kunc, R. R. Lowden, E. Lara-Curzio, R. Fredette, J. Ormerod, T. A. Lograsso, and M. P. Paranthaman, "Big Area Additive Manufacturing of High Performance Bonded NdFeB Magnets," Scientific Reports, vol. 6, no. October, pp. 1-7, 2016.

[168] S. J. Leigh, C. P. Purssell, D. R. Billson, and D. A. Hutchins, "Using a magnetite/thermoplastic composite in 3D printing of direct replacements for commercially available flow sensors," Smart Materials and Structures, vol. 23, no. 9, 2014.

[169] H. Watschke, K. Hilbig, and T. Vietor, "Design and characterization of electrically conductive structures additively manufactured by material extrusion," Applied Sciences (Switzerland), vol. 9, no. 4, pp. 1-25, 2019.

[170] S. J. Leigh, R. J. Bradley, C. P. Purssell, D. R. Billson, and D. A. Hutchins, "A Simple, Low-Cost Conductive Composite Material for 3D Printing of Electronic Sensors," PLoS ONE, vol. 7, no. 11, pp. 1-6, 2012.

[171] W. Lang, F. Jakobs, E. Tolstosheeva, H. Sturm, A. Ibragimov, A. Kesel, D. Lehmhus, and U. Dicke, "From embedded sensors to sensorial materials - The road to function scale integration," Sensors and Actuators, A: Physical, vol. 171, no. 1, pp. 3-11, 2011. [Online]. Available: http://dx.doi.org/10.1016/j.sna.2011.03.061

[172] M. Maurizi, J. Slavič, F. Cianetti, M. Jerman, J. Valentinčič, A. Lebar, and M. Boltežar, "Dynamic measurements using FDM 3D-printed embedded strain sensors," Sensors (Switzerland), vol. 19, no. 12, pp. 1-15, 2019.

[173] A. Dijkshoorn, P. Neuvel, S. Stramigioli, and G. Krijnen, "In-Situ Monitoring of Layer-Wise Fabrication by Electrical Resistance Measurements in 3D Printing," Proceedings of IEEE Sensors, 2020.

[174] J. R. McGhee, M. Sinclair, D. J. Southee, and K. G. U. Wijayantha, "Strain sensing characteristics of 3D-printed conductive plastics," Electronics Letters, vol. 54, no. 9, pp. 570-572, 2018.

[175] Z. Abass, W. Meng, S. Q. Xie, and Z. Zhang, "A robust, practical upper limb electromyography interface using dry 3D printed electrodes," IEEE/ASME International Conference on Advanced Intelligent Mechatronics, AIM, vol. 2019July, pp. 453-458, 2019.

[176] B. Hampel, S. Monshausen, and M. Schilling, "Properties and applications of electrically conductive thermoplastics for additive manufacturing of sensors," Technisches Messen, vol. 84, no. 9, pp. 593-599, 2017.

[177] P. Wiebe, P. Beierle, H. C. Shao, B. Gergely, A. F. Starace, and H. Batelaan, "Performance of plastic electron optics components fabricated using a 3D printer," Ultramicroscopy, vol. 205, pp. 70-74, 2019.

[178] M. J. Kim, M. A. Cruz, S. Ye, A. L. Gray, G. L. Smith, N. Lazarus, C. J. Walker, H. H. Sigmarsson, and B. J. Wiley, "One-step electrodeposition of copper on conductive 3D printed objects," Additive Manufacturing, vol. 27, no. November 2018, pp. 318-326, 2019. [Online]. Available: https://doi.org/10.1016/j.addma.2019.03.016 
[179] S. H. Ahn, M. Montero, D. Odell, S. Roundy, and P. K. Wright, "Anisotropic material properties of fused deposition modeling ABS," Rapid Prototyping Journal, vol. 8, no. 4, pp. 248-257, 2002.

[180] H. Prajapati, D. Ravoori, R. L. Woods, and A. Jain, "Measurement of anisotropic thermal conductivity and inter-layer thermal contact resistance in polymer fused deposition modeling (FDM)," Additive Manufacturing, vol. 21, no. February, pp. 84-90, 2018. [Online]. Available: https: //doi.org/10.1016/j.addma.2018.02.019

[181] M. V. Patton, P. Ryan, T. Calascione, N. Fischer, A. Morgenstern, N. Stenger, and B. B. Nelson-Cheeseman, "Manipulating magnetic anisotropy in fused filament fabricated parts via macroscopic shape, mesoscopic infill orientation, and infill percentage," Additive Manufacturing, vol. 27, no. November 2018, pp. 482-488, 2019. [Online]. Available: https: //doi.org/10.1016/j.addma.2019.03.026

[182] J. Zhang, B. Yang, F. Fu, F. You, X. Dong, and M. Dai, "Resistivity and Its Anisotropy Characterization of 3D-Printed Acrylonitrile Butadiene Styrene Copolymer (ABS)/Carbon Black (CB) Composites," Applied Sciences, vol. 7, no. 1 , p. $20,2017$.

[183] L. Truman, E. Whitwam, B. B. Nelson-Cheeseman, and L. J. Koerner, "Conductive 3D printing: resistivity dependence upon infill pattern and application to EMI shielding," Journal of Materials Science: Materials in Electronics, no. 0123456789, 2020. [Online]. Available: https://doi.org/10.1007/ s10854-020-03965-9

[184] S. Lin, L. Xia, G. Ma, S. Zhou, and Y. M. Xie, "A maze-like path generation scheme for fused deposition modeling," International Journal of Advanced Manufacturing Technology, vol. 104, no. 1-4, pp. 1509-1519, 2019.

[185] P. P. L. Regtien, F. van der Heijden, M. J. Korsten, and W. Olthuis, Meurement Science for Engineers. Kogan Page Science, 2004.

[186] X. Aeby, R. van Dommelen, and D. Briand, "Fully FDM 3D Printed Flexible Capacitive and Resistive Transducers," 2019 20th International Conference on Solid-State Sensors, Actuators and Microsystems 8 Eurosensors XXXIII (TRANSDUCERS \& EUROSENSORS XXXIII), no. June, pp. 2440-2443, 2019.

[187] M. Mohammadizadeh, I. Fidan, M. Allen, and A. Imeri, "Creep behavior analysis of additively manufactured fiber-reinforced components," International Journal of Advanced Manufacturing Technology, vol. 99, no. 5-8, pp. 1225-1234, 2018.

[188] K. V. Niaza, F. S. Senatov, A. Stepashkin, N. Y. Anisimova, and M. V. Kiselevsky, "Long-Term Creep and Impact Strength of Biocompatible 3D-Printed PLA-Based Scaffolds," Nano Hybrids and Composites, vol. 13, pp. 15-20, 2017.

[189] D. Coleman, M. Khalid, and X. Tan, "Temperature-compensation of 3D-printed polymer-based strain gauge," PROCEEDINGS OF SPIE, no. March, p. 20, 2019.

[190] H. T. Banks, "A brief review of some approaches to hysteresis in viscoelastic polymers," Nonlinear Analysis, Theory, Methods and Applications, vol. 69, no. 3, pp. 807-815, 2008. 
[191] J. Shintake, E. Piskarev, S. H. Jeong, and D. Floreano, "Ultrastretchable Strain Sensors Using Carbon Black-Filled Elastomer Composites and Comparison of Capacitive Versus Resistive Sensors," Advanced Materials Technologies, vol. 3, no. 3, pp. 1-8, 2018.

[192] V. Hassani, T. Tjahjowidodo, and T. N. Do, "A survey on hysteresis modeling, identification and control," Mechanical Systems and Signal Processing, vol. 49, no. 1-2, pp. 209-233, 2014. [Online]. Available: http://dx.doi.org/10.1016/j.ymssp.2014.04.012

[193] K. Kuhnen, "Modelling, identification, and compensation of complex hysteretic and $\log (\mathrm{t})$-type creep nonlinearities," Control and Intelligent Systems, vol. 33, no. 2, pp. 134-147, 2005.

[194] — - "Modeling, identification and compensation of complex hysteretic nonlinearities: A modified prandtl-ishlinskii approach," European Journal of Control, vol. 9, no. 4, pp. 407-418, 2003.

[195] A. Oliveri, F. Stellino, G. Caluori, M. Parodi, and M. Storace, "Open-Loop Compensation of Hysteresis and Creep Through a Power-Law Circuit Model," IEEE Transactions on Circuits and Systems I: Regular Papers, vol. 63, no. 3, pp. 413-422, 2016.

[196] M. Schouten, D. Kosmas, and G. Krijnen, "Hysteresis Compensation of 3D Printed Sensors Using a Power Law Model for Various Input Signals," Proceedings of IEEE Sensors, vol. 2020-Octob, no. 3, pp. 1-4, 2020.

[197] M. Ntagios, H. Nassar, A. Pullanchiyodan, W. T. Navaraj, and R. Dahiya, "Robotic Hands with Intrinsic Tactile Sensing via 3D Printed Soft Pressure Sensors," Advanced Intelligent Systems, no. October, p. 1900080, 2019.

[198] K. Li, H. Wei, W. Liu, H. Meng, P. Zhang, and C. Yan, "3D printed stretchable capacitive sensors for highly sensitive tactile and electrochemical sensing," Nanotechnology, vol. 29, no. 18, 2018.

[199] B. Chou, J. S. Park, and W. S. Kim, "3D printed inductor designs decorated with silver nano ink," 2015 IEEE Nanotechnology Materials and Devices Conference, NMDC 2015, pp. 1-2, 2016.

[200] M. Kisic, N. Blaz, L. Zivanov, and M. Damnjanovic, "Elastomer based force sensor fabricated by $3 \mathrm{D}$ additive manufacturing," AIP Advances, vol. 10, no. 1, pp. 1-5, 2020. [Online]. Available: https://doi.org/10.1063/1.5130065

[201] S. Y. Wu, C. Yang, W. Hsu, and L. Lin, "RF wireless lc tank sensors fabricated by 3D additive manufacturing," 2015 Transducers - 2015 18th International Conference on Solid-State Sensors, Actuators and Microsystems, TRANSDUCERS 2015, pp. 2208-2211, 2015.

[202] D. Staudenmann, K. Roeleveld, D. F. Stegeman, and J. H. van Dieen, "Methodological aspects of SEMG recordings for force estimation-a tutorial and review," J Electromyogr Kinesiol, vol. 20, no. 3, pp. 375-387, 2010. [Online]. Available: http://www.ncbi.nlm.nih.gov/pubmed/19758823

[203] A. E. Schultz and T. A. Kuiken, "Neural interfaces for control of upper limb prostheses: the state of the art and future possibilities," PMER, vol. 3, no. 1, pp. $55-67,2011$. 
[204] S. B. Godfrey, A. Ajoudani, M. Catalano, G. Grioli, and A. Bicchi, "A synergydriven approach to a myoelectric hand," IEEE International Conference on Rehabilitation Robotics, pp. 11-16, 2013.

[205] G. Postolache, A. Paiva, H. Carvalho, A. Catarino, O. Postolache, and G. Postolache, "Development of Dry Textile Electrodes for Electromiography," Sensing Technology (ICST), 2015 9th International Conference on, pp. 447-451, 2015.

[206] L. Bareket, L. Inzelberg, D. Rand, M. David-Pur, D. Rabinovich, B. Brandes, and Y. Hanein, "Temporary-tattoo for long-term high fidelity biopotential recordings," Scientific Reports, vol. 6, no. May, pp. 1-8, 2016.

[207] J. G. Webster and J. W. Clark, Medical instrumentation : application and design, 4th ed. Hoboken, NJ: John Wiley \& Sons, 2010.

[208] C. J. D. Luca, "Surface Electromyography: Detection and Recording," 2002.

[209] H. J. Hermens, B. Freriks, C. Disselhorst-Klug, and G. Rau, "Development of recommendations for SEMG sensors and sensor placement procedures," $J$ Electromyogr Kinesiol, vol. 10, no. 5, pp. 361-374, 2000. [Online]. Available: http://www.ncbi.nlm.nih.gov/pubmed/11018445

[210] L. Xie, G. Yang, L. Xu, F. Seoane, Q. Chen, and L. Zheng, "Characterization of dry biopotential electrodes," Conf Proc IEEE Eng Med Biol Soc, vol. 2013, pp. 1478-1481, 2013. [Online]. Available: http://www.ncbi.nlm.nih.gov/pubmed/24109978

[211] P. Salvo, R. Raedt, E. Carrette, D. Schaubroeck, J. Vanfleteren, and L. Cardon, "A 3D printed dry electrode for ECG/EEG recording," Sensors and Actuators A: Physical, vol. 174, pp. 96-4247, 2012.

[212] N. Meziane, J. G. Webster, M. Attari, and A. J. Nimunkar, "Dry electrodes for electrocardiography," Physiol Meas, vol. 34, no. 9, pp. R47-69, 2013. [Online]. Available: http://www.ncbi.nlm.nih.gov/pubmed/24137716

[213] Y. H. Chen, M. de Beeck, L. Vanderheyden, E. Carrette, V. Mihajlovic, K. Vanstreels, B. Grundlehner, S. Gadeyne, P. Boon, and C. Van Hoof, "Soft, comfortable polymer dry electrodes for high quality ECG and EEG recording," Sensors (Basel), vol. 14, no. 12, pp. 23 758-23780, 2014. [Online]. Available: http://www.ncbi.nlm.nih.gov/pubmed/25513825

[214] C. Y. Chen, C. L. Chang, C. W. Chang, S. C. Lai, T. F. Chien, H. Y. Huang, J. C. Chiou, and C. H. Luo, "A low-power bio-potential acquisition system with flexible PDMS dry electrodes for portable ubiquitous healthcare applications," Sensors (Basel), vol. 13, no. 3, pp. 3077-3091, 2013. [Online]. Available: http://www.ncbi.nlm.nih.gov/pubmed/23459390

[215] H. Manabe, M. Fukumoto, and T. Yagi, "Conductive rubber electrodes for earphone-based eye gesture input interface," Personal and Ubiquitous Computing, vol. 19, no. 1, pp. 143-4909, 2015.

[216] C. Pylatiuk, M. Muller-Riederer, A. Kargov, S. Schulz, O. Schill, M. Reischl, and G. Bretthauer, "Comparison of surface EMG monitoring electrodes for long-term use in rehabilitation device control." IEEE, 2009, Conference Proceedings, pp. 300-3041424437881. 
[217] Y. M. Chi, T. P. Jung, and G. Cauwenberghs, "Dry-contact and noncontact biopotential electrodes: methodological review," IEEE Rev Biomed Eng, vol. 3, pp. 106-119, 2010. [Online]. Available: http: //www.ncbi.nlm.nih.gov/pubmed/22275204

[218] K. P. Hoffmann, R. Ruff, and W. Poppendieck, "Long-term characterization of electrode materials for surface electrodes in biopotential recording," Conf Proc IEEE Eng Med Biol Soc, vol. 1, pp. 2239-2242, 2006. [Online]. Available: http://www.ncbi.nlm.nih.gov/pubmed/17945701

[219] TMS-International B.V, "Technical Specifications Refa Rev4," Tech. Rep., 2017.

[220] J. P. Swensen, L. U. Odhner, B. Araki, and A. M. Dollar, "Printing threedimensional electrical traces in additive manufactured parts for injection of low melting temperature metals," Journal of Mechanisms and Robotics, vol. 7, no. 2, pp. 1-10, 2015.

[221] M. Vatani, Y. Lu, E. D. Engeberg, and J.-W. Choi, "Combined 3D printing technologies and material for fabrication of tactile sensors," International Journal of Precision Engineering and Manufacturing, vol. 16, no. 7, pp. 1375-1383, 2015.

[222] S.-Y. Wu, C. Yang, W. Hsu, and L. Lin, "3D-printed microelectronics for integrated circuitry and passive wireless sensors," Microsystems $\& 3$ Nanoengineering, vol. 1, 2015.

[223] M. K. Welleweerd, "3D printing a three degree of freedom force sensor," Thesis, University of Twente, 2017. [Online]. Available: http://essay.utwente.nl/72039/

[224] J. J. Adams, E. B. Duoss, T. F. Malkowski, M. J. Motala, B. Y. Ahn, R. G. Nuzzo, J. T. Bernhard, and J. A. Lewis, "Conformal printing of electrically small antennas on three-dimensional surfaces," Advanced Materials, vol. 23, no. 11, pp. 1335-1340, 2011.

[225] J. Odent, T. J. Wallin, W. Pan, K. Kruemplestaedter, R. F. Shepherd, and E. P. Giannelis, "Highly Elastic, Transparent, and Conductive 3D-Printed Ionic Composite Hydrogels," Advanced Functional Materials, vol. 27, no. 33, pp. 1-10, 2017.

[226] Polymaker. PolyFlex. [Online]. Available: http://www.polymaker.com/shop/ polyflex/

[227] Diabase Engineering. X60 Ultra-Flexible Filament - White. [Online]. Available: https://flexionextruder.com/shop/x60-ultra-flexible-filament-white/

[228] Lay-Filaments. 4D Meta material: The PoroLAY series. [Online]. Available: http://lay-filaments.com

[229] Palmiga Innovation. Material info for PI-ETPU 95-250 Carbon Black the conductive and flexible 3D printing filament. [Online]. Available: http://rubber3dprinting.com/pi-etpu-95-250-carbon-black/

[230] S. H. Munson-McGee, "Estimation of the critical concentration in an anisotropic percolation network," Physical Review B, vol. 43, no. 4, p. 3331, 1991.

[231] SENIAM. [Online]. Available: www.seniam.org 
[232] The MathWorks, Inc. Discrete-time, direct-form II filter. [Online]. Available: https://mathworks.com/help/signal/ref/dfilt.df2.html

[233] A. Dijkshoorn, M. Schouten, G. Wolterink, R. Sanders, and G. Krijnen, "Characterizing the Electrical Properties of Anisotropic, 3D-Printed Conductive Sheets," FLEPS 2019 - IEEE International Conference on Flexible and Printable Sensors and Systems, Proceedings, pp. 2019-2021, 2019.

[234] H. Daley, K. Englehart, L. Hargrove, and U. Kuruganti, "High density electromyography data of normally limbed and transradial amputee subjects for multifunction prosthetic control," Journal of Electromyography and Kinesiology, vol. 22, no. 3, pp. 478-484, 2012. [Online]. Available: http://dx.doi.org/10.1016/j.jelekin.2011.12.012

[235] G. Li, A. E. Schultz, and T. A. Kuiken, "Quantifying pattern recognition- based myoelectric control of multifunctional transradial prostheses," IEEE Transactions on Neural Systems and Rehabilitation Engineering, vol. 18, no. 2, pp. 185192, 2010.

[236] P. H. Veltink, H. Kortier, and H. M. Schepers, "Sensing power transfer between the human body and the environment," IEEE Transactions on Biomedical Engineering, vol. 56, no. 6, pp. 1711-1718, 2009.

[237] J. Yin, V. J. Santos, and J. D. Posner, "Bioinspired flexible microfluidic shear force sensor skin," Sensors and Actuators, A: Physical, vol. 264, pp. 289-297, 2017. [Online]. Available: http://dx.doi.org/10.1016/j.sna.2017.08.001

[238] S. SHIMAWAKI and N. SAKAI, "Quasi-static Deformation Analysis of a Human Finger using a Three-dimensional Finite Element Model Constructed from CT Images," Journal of Environment and Engineering, vol. 2, no. 1, pp. 56-63, 2007.

[239] C. Tawk and G. Alici, "Finite element modeling in the design process of 3D printed pneumatic soft actuators and sensors," Robotics, vol. 9, no. 9, 2020.

[240] M. Al-Rubaiai, R. Tsuruta, U. Gandhi, C. Wang, and X. Tan, "A 3D-printed stretchable strain sensor for wind sensing," Smart Materials and Structures, vol. 28 , no. 8, p. 84001, 2019.

[241] A. Rusinek, G. Robles, and A. Arias, "Conductive 3D Printed PLA Composites: on the interplay of mechanical, electrical and thermal behaviours," Composite Structures, p. 113744, 2021. [Online]. Available: https://doi.org/10.1016/j.compstruct.2021.113744

[242] A. Tognetti, F. Lorussi, G. D. Mura, N. Carbonaro, M. Pacelli, R. Paradiso, and D. D. Rossi, "New generation of wearable goniometers for motion capture systems," Journal of NeuroEngineering and Rehabilitation, vol. 11, no. 1, pp. $1-17,2014$.

[243] R. A. Brookhuis, H. Droogendijk, M. J. De Boer, R. G. Sanders, T. S. Lammerink, R. J. Wiegerink, and G. J. Krijnen, "Six-axis force-torque sensor with a large range for biomechanical applications," Journal of Micromechanics and Microengineering, vol. 24, no. 3, 2014. 
[244] R. Xie, Y. Xie, C. R. López-Barrón, K.-Z. Gao, and N. J. Wagner, "UltraStretchable Conductive Iono-Elastomer And Motion Strain Sensor System Developed Therefrom," Technology And Innovation, vol. 19, no. 3, pp. 613-626, 2018 .

[245] R. A. Romeo, F. Cordella, L. Zollo, D. Formica, P. Saccomandi, E. Schena, G. Carpino, A. Davalli, R. Sacchetti, and E. Guglielmelli, "Development and preliminary testing of an instrumented object for force analysis during grasping," Proceedings of the Annual International Conference of the IEEE Engineering in Medicine and Biology Society, EMBS, vol. 2015-Novem, pp. 6720-6723, 2015.

[246] F. Cordella, F. Taffoni, L. Raiano, G. Carpino, M. Pantoni, L. Zollo, E. Schena, E. Guglielmelli, and D. Formica, "Design and development of a sensorized cylindrical object for grasping assessment," Proceedings of the Annual International Conference of the IEEE Engineering in Medicine and Biology Society, EMBS, vol. 2016-Octob, pp. 3366-3369, 2016.

[247] R. A. Brookhuis, R. G. Sanders, K. Ma, T. S. Lammerink, M. J. De Boer, G. J. Krijnen, and R. J. Wiegerink, "Miniature large range multi-axis forcetorque sensor for biomechanical applications," Journal of Micromechanics and Microengineering, vol. 25, no. 2, 2015.

[248] L. Beccai, S. Roccella, L. Ascari, P. Valdastri, A. Sieber, M. C. Carrozza, and P. Dario, "Development and experimental analysis of a soft compliant tactile microsensor for anthropomorphic artificial hand," IEEE/ASME Transactions on Mechatronics, vol. 13, no. 2, pp. 158-168, 2008.

[249] L. Viry, A. Levi, M. Totaro, A. Mondini, V. Mattoli, B. Mazzolai, and L. Beccai, "Flexible three-axial force sensor for soft and highly sensitive artificial touch," Advanced Materials, vol. 26, no. 17, pp. 2659-2664, 2014.

[250] F. Hammond and R. Wood, "Toward a Modular Soft Sensor - Embedded Glove for," 2014 IEEE/RSJ International Conference on Intelligent Robots and Systems, no. Iros, pp. 4000-4007, 2014.

[251] G. Wolterink, R. Sanders, B. J. van Beijnum, P. Veltink, and G. Krijnen, "A 3dprinted soft fingertip sensor for providing information about normal and shear components of interaction forces," Sensors, vol. 21, no. 13, pp. 1-13, 2021.

[252] "Shallow Neural Network Time-Series Prediction and Modeling," (R2021a), The MathWorks, Inc., 2021. [Online]. Available: https://mathworks.com/help/ deeplearning/gs/neural-network-time-series-prediction-and-modeling.html

[253] D. Kosmas, M. Schouten, and G. Krijnen, "Hysteresis Compensation of 3D Printed Sensors by a Power Law Model with Reduced Parameters," in FLEPS 2020 - IEEE International Conference on Flexible and Printable Sensors and Systems, Proceedings, 2020.

[254] "Matlab optimization toolbox," Version 8.4 (R2019b), The MathWorks, Inc., 2021. [Online]. Available: https://nl.mathworks.com/products/ global-optimization.html

[255] "Matlab GlobalSearch," (R2021a), The MathWorks, Inc., 2021. [Online]. Available: https://nl.mathworks.com/help/gads/globalsearch.html 
[256] "fmincon," (R2021a), The MathWorks, Inc., 2021. [Online]. Available: https://mathworks.com/help/optim/ug/fmincon.html

[257] M. Parodi, M. Storace, and S. Cincotti, "A PWL ladder circuit which exhibits hysteresis," International Journal of Circuit Theory and Applications, vol. 22, no. 6, pp. 513-526, 1994.

[258] E. R. Serina, C. D. Mote, and D. Rempel, "Force response of the fingertip pulp to repeated compression - Effects of loading rate, loading angle and anthropometry," Journal of Biomechanics, vol. 30, no. 10, pp. 1035-1040, 1997.

[259] P. Leonardo, "Inverse determination of viscoelastic properties of human fingertip skin," RMZ - Materials and Geoenvironment, vol. 57, no. 1, pp. 1-16, 2010.

[260] E. Repnik, U. Puh, N. Goljar, M. Munih, and M. Mihelj, "Using Inertial Measurement Units and Electromyography to Quantify Movement during Action Research Arm Test Execution," Sensors, vol. 18, no. 9, p. 2767, 2018.

[261] G. Averta, C. Della Santina, E. Battaglia, F. Felici, M. Bianchi, and A. Bicchi, "Unvealing the principal modes of human upper limb movements through functional analysis," Frontiers Robotics AI, vol. 4, no. AUG, pp. 1-12, 2017.

[262] P. MacEira-Elvira, T. Popa, A. C. Schmid, and F. C. Hummel, "Wearable technology in stroke rehabilitation: Towards improved diagnosis and treatment of upper-limb motor impairment," Journal of NeuroEngineering and Rehabilitation, vol. 16, no. 1, 2019.

[263] C. Fang, B. He, Y. Wang, J. Cao, and S. Gao, "EMG-centered multisensory based technologies for pattern recognition in rehabilitation: State of the art and challenges," Biosensors, vol. 10, no. 8, 2020.

[264] N. Taubert, J. St. Amand, P. Kumar, L. Gizzi, and M. A. Giese, Reactive Hand Movements from Arm Kinematics and EMG Signals Based on Hierarchical Gaussian Process Dynamical Models. Springer International Publishing, 2020, vol. 12396 LNCS. [Online]. Available: http://dx.doi.org/10.1007/978-3-030-61609-0_11

[265] H. G. Kortier, "Assessment of hand kinematics and interactions with the environment," Ph.D. dissertation, University of Twente, Netherlands, feb 2018.

[266] M. Bianchi, R. Haschke, G. Büscher, S. Ciotti, N. Carbonaro, and A. Tognetti, "A multi-modal sensing glove for human manual-interaction studies," Electronics (Switzerland), vol. 5, no. 3, pp. 1-15, 2016.

[267] StretchSense, "Mocap Pro Supersplay." [Online]. Available: https:// stretchsense.com/

[268] Xsens, "Xsens gloves by manus." [Online]. Available: https://www.xsens.com/ products/xsens-gloves-by-manus

[269] C. Haarman and M. Tschiersky, "D7.2 (D27) - Unilateral, body-mounted, robotic arm," European Union's Horizon 2020 research and innovation programme Grant Agreement No. 688857, Tech. Rep. Ares(2019)2837516, 2019. [Online]. Available: https://cordis.europa.eu/project/id/688857/results 
[270] M. R. Al-Mulla, F. Sepulveda, and M. Colley, "A review of non-invasive techniques to detect and predict localised muscle fatigue," Sensors, vol. 11, no. 4, pp. 3545-3594, 2011.

[271] J. Taborri, J. Keogh, A. Kos, A. Santuz, A. Umek, C. Urbanczyk, E. van der Kruk, and S. Rossi, "Sport biomechanics applications using inertial, force, and EMG sensors: A literature overview," Applied Bionics and Biomechanics, vol. 2020, 2020.

[272] G. Salvietti, L. Franco, M. Tschiersky, G. Wolterink, M. Bianchi, A. Bicchi, F. Barontini, M. Catalano, G. Grioli, M. Poggiani, S. Rossi, and D. Prattichizzo, "Integration of a Passive Exoskeleton and a Robotic Supernumerary Finger for Grasping Compensation in Chronic Stroke Patients: The SoftPro Wearable System," Frontiers in Robotics and AI, vol. 8, no. June, pp. 1-9, 2021.

[273] G. Wolterink, A. Umrani, S. Martijn, R. Sanders, and G. Krijnen, "3D-Printed Calorimetric Flow Sensor," in Proceedings of IEEE Sensors, no. 2, 2020, pp. 3-5.

[274] L. R. Lopes, A. F. Silva, and O. S. Carneiro, "Multi-material 3D printing: The relevance of materials affinity on the boundary interface performance," Additive Manufacturing, vol. 23, no. July, pp. 45-52, 2018.

[275] E. Cantu, T. Fapanni, G. Giorgi, C. Narduzzi, E. Sardini, M. Serpelloni, and S. Tonello, "Printed Multi-EMG Electrodes on the 3D Surface of an Orthosis for Rehabilitation: a Feasibility Study," IEEE Sensors Journal, vol. 21, no. 13, pp. 14 407-14 417, 2021.

[276] D. Pani, A. Achilli, A. Spanu, A. Bonfiglio, M. Gazzoni, and A. Botter, "Validation of Polymer-Based Screen-Printed Textile Electrodes for Surface EMG Detection," IEEE Transactions on Neural Systems and Rehabilitation Engineering, vol. 27, no. 7, pp. 1370-1377, 2019.

[277] D. Zymelka, K. Togashi, T. Takeshita, T. Yamashita, and T. Kobayashi, "Practical evaluation of printed strain sensors based on long-term static strain measurements," Sensors, vol. 21, no. 14, pp. 1-13, 2021.

[278] M. Hinderdael, Z. Jardon, M. Lison, D. de Baere, W. Devesse, M. Strantza, and P. Guillaume, "Proof of concept of integrated load measurement in 3D printed structures," Sensors (Switzerland), vol. 17, no. 2, pp. 1-12, 2017.

[279] M. A. Saleh, R. Kempers, and G. W. Melenka, "3D printed continuous wire polymer composites strain sensors for structural health monitoring," Smart $M a$ terials and Structures, vol. 28, no. 10, 2019.

[280] E. Burdet, D. W. Franklin, and T. E. Milner, Human robotics: neuromechanics and motor control. MIT press, 2013.

[281] M. K. Haugland, J. A. Hoffer, and T. Sinkjær, "Skin Contact Force Information in Sensory Nerve Signals Recorded by Implanted Cuff Electrodes," IEEE Transactions on Rehabilitation Engineering, vol. 2, no. 1, pp. 18-28, 1994. 


\section{Publications}

\section{Journal publications}

G. Wolterink, R. Sanders, B.J. van Beijnum, P. Veltink, G. Krijnen, A 3D printed Soft Fingertip Sensor for Providing Information about Normal and Shear Components of Interaction Forces, Sensors 21 (13), 4271, 2021

M. Schouten, G. Wolterink, A. Dijkshoorn, D. Kosmas, S. Stramigioli, G. Krijnen, A review of extrusion-based 3d printing for the fabrication of electro-and biomechanical sensors, IEEE Sensors Journal, 2021

G. Salvietti, L. Franco, M. Tschiersky, G. Wolterink, M. Bianchi, A. Bicchi, F. Barontini, M. Catalano, G. Grioli, M. Poggiani, S. Rossi, D. Prattichizzo Integration of a Passive Exoskeleton and a Robotic Supernumerary Finger for Grasping Compensation in Chronic Stroke Patients: The SoftPro Wearable System, Frontiers in Robotics and AI 8, 2021

M. Bhagubai*, G. Wolterink*, A. Schwarz, J. Held, B.J. Van Beijnum, P. Veltink Quantifying Pathological Synergies in the Upper Extremity of Stroke Subjects With the Use of Inertial Measurement Units: A Pilot Study, IEEE journal of translational engineering in health and medicine, 2020, *Shared first authorship,

R. Edgar, E.J. Trip, G. Wolterink, P. Veltink, J.J.H. Beck, New methods for the monitoring of nocturnal erections, International Journal of Impotence Research, $1-7,2020$

A. Dijkshoorn, M. Schouten, G. Wolterink, R. Sanders, S. Stramigioli, G. Krijnen, Characterizing the electrical properties of anisotropic, 3D printed conductive sheets for sensor applications, IEEE Sensors Journal 20 (23), 14218-14227, 2020 
A. Schwarz, M. Bhagubai, G. Wolterink, J. Held, A.R. Luft, P. Veltink, Assessment of upper limb movement impairments after stroke using wearable inertial sensing, Sensors 20 (17), 4770, 2020

G. Wolterink, P. Dias, R. Sanders, F. Muijzer, B.J. van Beijnum, P Veltink, G. Krijnen, Development of soft sEMG sensing structures using 3D printing technologies, Sensors 20 (15), 4292, 2020

A. Dijkshoorn, P. Werkman, M. Welleweerd, G. Wolterink, B. Eijking, J. Delamare, R. Sanders, G. Krijnen, Embedded sensing: integrating sensors in 3-D printed structures, Journal of Sensors and Sensor Systems 7 (1), 169-181, 2018

\section{Submitted}

G. Wolterink, D. Kosmas, M. Schouten, B.J. van Beijnum, P. Veltink, G. Krijnen, Evaluation of A 3D printed Soft Sensor for Measuring Fingertip Interaction Forces, Manuscript submitted for publication

\section{Conference proceedings}

G. Wolterink, A. Umrani, M. Schouten, R. Sanders, G. Krijnen, 3D printed Calorimetric Flow Sensor, 2020 IEEE Sensors, 1-4, 2020

L. Franco, M. Tschiersky, G. Wolterink, F. Barontini, M. Poggiani, M. Catalano, G. Grioli, M. Bianchi, A. Bicchi, S. Rossi, D. Prattichizzo, G. Salvietti, The SoftPro Wearable System for Grasp Compensation in Stroke Patients, International Symposium on Wearable Robotics, 363-367, 2020

G. Wolterink, R. Sanders, G. Krijnen, A flexible, three material, 3D printed, shear force sensor for use on finger tips, 2019 IEEE SENSORS, 1-4, 2019

A. Dijkshoorn, M. Schouten, G. Wolterink, R. Sanders, G. Krijnen, Characterizing the electrical properties of anisotropic, 3D printed conductive sheets, 2019 IEEE International Conference on Flexible and Printable Sensors and Systems (FLEPS), 2019 
G. Wolterink, R. Sanders, G. Krijnen, Thin, flexible, capacitive force sensors based on anisotropy in 3D printed structures, 2018 IEEE SENSORS, 1-4, 2018

G. Wolterink, R. Sanders, F. Muijzer, B.J. van Beijnum, G. Krijnen, 3D printing soft sEMG sensing structures, 2017 IEEE SENSORS, 1-3, 2017

P. Werkman, M. Welleweerd, G. Wolterink, J. Delamare, R. Sanders, G. Krijnen, A. Dijkshoorn, Embedded Sensing: Making the best of 3D printed sensors, 18th International Conference on Sensors and Measurement Technology 2017, 2017 


\section{About the author}

Gerhard Jan Willem (Gerjan) Wolterink was born on the $13^{\text {th }}$ of August, 1992 in Almelo, The Netherlands, and raised in Hellendoorn. He received his high school diploma in 2011 at the Pius X College in Almelo and started his bachelor study Biomedical Engineering at the University of Twente. He obtained his bachelor's degree in 2014 on the topic of using mechanomyography as a control interface for an arm support. After receiving his bachelor's degree, Gerjan started his master Biomedical Engineering at the University of Twente with the specialization in Neural and Motor

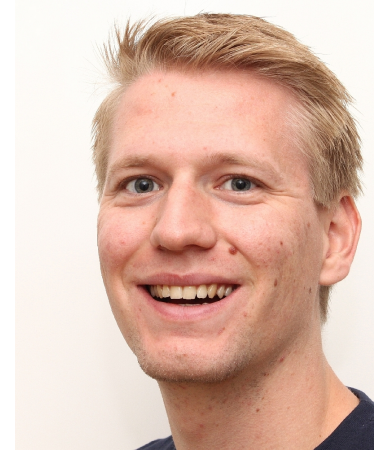
Systems. He followed an internship on prototyping and evaluating sensors for biomechanical feedback at Össur in Reykjavik, Iceland. Back at the University of Twente he started his master thesis within the SoftPro project under supervision of prof. dr. ir. Gijs Krijnen. In 2017 he received his master's degree with his thesis on 3D printing of soft surface electromyography structures. In the same year Gerjan started his $\mathrm{PhD}$ research in both the Robotics and Mechatronics and the Biomedical Signals and Systems group at the University of Twente under the supervision of prof. dr. ir. Gijs Krijnen and prof. dr. ir. Peter Veltink. After his PhD trajectory he continues the research on 3D printed sensors within the 4TU Dutch Soft Robotics Consortium as a postdoctoral researcher at the Robotics and Mechatronics group of the University of Twente. 


\section{Dankwoord}

Dit proefschrift was niet mogelijk geweest zonder de hulp, steun en (gezonde) afleiding van vele mensen. In dit laatste deel van dit proefschrift wil ik dan ook van de gelegenheid gebruik maken om hen te bedanken.

Als eerste wil ik mijn (co-)promotoren Gijs, Peter en Bert-Jan bedanken voor de kans die jullie mij hebben gegeven voor het aangaan van dit promotietraject en voor alle ondersteuning die jullie mij hierin hebben gegeven. Gijs, al tijdens mijn masteropdracht was het fijn om met jou samen te werken. Jij wist mij uit te dagen en jouw oog voor detail heeft vele papers en ook dit proefschrift verbeterd. Ik ben blij dat wij de samenwerking door hebben kunnen zetten tijdens en na mijn promotie. Peter, bij jou en bij jouw vakgroep voelde ik mij met mijn BMT achtergrond op mijn plek door jouw grote kennis van zowel de medische en technische onderwerpen. De samenwerkingen met clinici die jij hebt geïnitieerd zijn een grote motivatie geweest voor het werk in dit proefschrift. Bert-Jan, bedankt voor jouw bijdrage als co-promotor en het deelnemen aan de gezamenlijke meetingen met Gijs en Peter waar jij vaak aan het einde weer structuur in wist aan te brengen. Ook jouw hulp in de begeleiding van studenten werd zeer gewaardeerd en heb ook ik veel van geleerd.

This project was part of the SoftPro project. I would like to thank all members for the interesting, although sometimes too long, meetings and hospitality. My special thanks goes to Anne for all your help and hard work on the iHams studies and recruitment of the subjects. Jeremia, thank you for your support and feedback on this work. Frodo, bedankt voor al jouw hulp vanuit TMSi en later BSS en voor het fijne gezelschap tijdens de SoftPro meetings. Claudia, ik heb met veel plezier aan de MAS demonstrator gewerkt, wie weet kunnen we hier ooit nog eens mee verder. All people from the SoftPro Twente-team: Benjamin, Bert-Jan, Claudia, Ed, Freek, Frodo, Gijs, Herman, Martin, Peter, Stefan and Stefano, thank you for the good company during our trips and meetings, it was a pleasure to be a part of this team.

Martijn, de momenten dat het halve lab plakte van de appelstroop en honing 
maakte jij meer dan goed met jouw grote bereidheid om te helpen en jouw suggesties voor verbeteringen. Alexander, ook jij bent altijd bereid te helpen en zit vol goede ideeën. Op jou en Martijn kan ik altijd rekenen, daarom ben ik blij dat jullie mij ook als paranymphen bijstaan. Dimitris, I am happy another 3DPrinting enthousiast joined the NIFTy group, your enthusiasm and willingness to help makes you a great person to works with.

Dank aan alle andere collega's bij BSS en RAM voor de fijne werkomgeving en wat heb ik een geluk gehad om een lid te mogen zijn van The Best Office: Stefan, Maaike, Mourad, Frieda, Rob, Marcel (the founding father of FancyColourPlot.m), Hengameh, Martijn, Jornt, Toon, Elfi, Marieke en Robin, bedankt voor jullie gezelligheid en koffiemomentjes. All members from the NIFTy group, thank you for sharing your knowledge, feedback and help with troubleshooting. Jolanda en Sandra, bedankt voor al jullie hulp en regelwerk, waar zouden de vakgroepen zijn zonder jullie? Gerben, Hennie, Marcel en Sander bedankt voor jullie hulp en sorry voor mijn ongeduldigheid met de bestellingen. Ed, het was een waar genoegen om nog met jou te mogen samenwerken. Jij was een goede co-piloot in het Italiaanse verkeer en ik heb genoten van onze uitjes in Florence en Pisa. Pino, eigenlijk heeft elke promovendus een Pino nodig. Zonder jouw kennis, creativiteit en vooral morele ondersteuning was dit proefschrift nooit tot stand gekomen.

All students that helped me during my research: Ajinkya, Ameya, Amid, Bima, Claudia, Jacques, Jens, Jos, Maaike, Miguel, Nikhil, Pedro, Ralph. Thank you, it was a pleasure working with all of you and I have learned a lot from you as well. Miguel, it was a pleasure working with you on the iHams project, I was happy you wanted to stay a little longer after your graduation. Without you, Chapter 2 would not have been so extensive. I wish you all the best with your $\mathrm{PhD}$ position in Leuven.

Aan alle proefpersonen die mee hebben gedaan aan mijn onderzoeken, bedankt dat jullie de moeite en tijd hebben genomen om mij verder te helpen.

Ik wil ook graag mijn vrienden bedanken voor de nodige afleiding tijdens mijn promotietraject en mij te helpen even aan wat anders te denken en te ontspannen. Maarten, Patrick, Suzanne, Dennis en Djoey onze backpack weekenden waren altijd een heerlijk moment om er een lang weekend tussenuit te zijn, iets wat we snel weer een keer moeten gaan doen en niet te vergeten, een avondje foto's kijken. Ook de rest van de Aapjes App: Anouk, Arjan, Axel, Edwin, Eline, Ellen, Frank, Gerard, Irma, Jeroen, Jochem, Joost, Karin, Laura, Leonie, Marinko, Mathijs, Prescilla, Trienke en Vincent, ook al snapte niet iedereen waar ik mee bezig was, het is altijd gezellig en het is fijn om onderdeel te zijn van zo'n grote groep mensen die voor elkaar klaar staan. Djoey, jouw 
interesse in het onderzoek heb altijd zeer gewaardeerd.

Belinda, David, Maaike wat fijn dat we nog steeds na het afronden van onze studie als vrienden bij elkaar zijn. Al kunnen we niet meer elke pauze afspreken, het is mooi dat we elkaar nog steeds vaak zien en veel gezellige uitjes, weekendjes en etentjes samen hebben. Martin, we spreken elkaar door de afstand niet heel veel meer, maar als we bij elkaar zijn is het altijd weer gezellig.

Iedereen bij de Reggegroep en in het bijzonder de leden van de Hakwadagstam, de RSA en het leiding team Pieter en Maarten, bedankt voor de weekenden vol vermaak en nodige afleiding.

Lieve schoonfamilie Jos, Willy, Monique, Stefan, Jeanine en de jongens Joah en Lucas. Bij jullie voel ik mij altijd welkom, bedankt voor jullie gezelligheid en afleiding.

Pap en mam, jullie hebben mij als klein jongetje al veel bijgebracht over techniek en wetenschap. Heel erg bedankt voor alles wat jullie voor mij gedaan hebben, zonder jullie steun was ik nooit zover gekomen en was dit proefschrift er nooit geweest. Nick, ik ben altijd super trots op jouw werk, jouw gedrevenheid en enthousiasme is aanstekelijk en dat is voor mij ook een grote motivator om van dit proefschrift wat moois te maken. Pap, mam, Nick en Britt bedankt voor alle gezelligheid en afleiding thuis in Helledoorn en de weekendjes weg.

Lieve Esther, dankjewel voor al jouw steun, afleiding, thuiswerk gezelligheid, koffie en knuffels. Ik heb jou ongevraagd veel lastig gevallen en geklaagd over mijn proefschrift, sorry daarvoor. Maar dat luisteren heeft ook veel goeds gebracht in zowel dit proefschrift als mijn gemoedstoestand. Lieve Esther, hoe had ik dit ooit zonder jou moeten doen, ik kan niet zonder jou! 